\title{
Combining Statistical and Spectral Analysis Techniques in Network Traffic Anomaly Detection
}

\author{
by \\ Stevan Novakov, B. Eng. (CSE) \\ A thesis submitted to the Faculty of Graduate and Postdoctoral \\ Affairs in partial fulfillment of the requirements \\ for the degree of \\ Master of Applied Science \\ in \\ Electrical and Computer Engineering \\ Ottawa-Carleton Institute for Electrical and Computer Engineering \\ Department of Systems and Computer Engineering \\ Carleton University \\ Ottawa, Ontario, Canada K1S 5B6
}

C Copyright 2012, Stevan Novakov 
Library and Archives

Canada

Published Heritage

Branch

395 Wellington Street

Ottawa ON K1A ON4

Canada
Bibliothèque et

Archives Canada

Direction du

Patrimoine de l'édition

395 , rue Wellington

Ottawa ON K1A ON4

Canada
Your file Votre référence

ISBN: 978-0-494-91504-2

Our file Notre référence

ISBN: $978-0-494-91504-2$
NOTICE:

The author has granted a nonexclusive license allowing Library and Archives Canada to reproduce, publish, archive, preserve, conserve, communicate to the public by telecommunication or on the Internet, loan, distrbute and sell theses worldwide, for commercial or noncommercial purposes, in microform, paper, electronic and/or any other formats.

The author retains copyright ownership and moral rights in this thesis. Neither the thesis nor substantial extracts from it may be printed or otherwise reproduced without the author's permission.
AVIS:

L'auteur a accordé une licence non exclusive permettant à la Bibliothèque et Archives Canada de reproduire, publier, archiver, sauvegarder, conserver, transmettre au public par télécommunication ou par l'Internet, prêter, distribuer et vendre des thèses partout dans le monde, à des fins commerciales ou autres, sur support microforme, papier, électronique et/ou autres formats.

L'auteur conserve la propriété du droit d'auteur et des droits moraux qui protege cette thèse. $\mathrm{Ni}$ la thèse ni des extraits substantiels de celle-ci ne doivent être imprimés ou autrement reproduits sans son autorisation.
In compliance with the Canadian Privacy Act some supporting forms may have been removed from this thesis.

While these forms may be included in the document page count, their removal does not represent any loss of content from the thesis.
Conformément à la loi canadienne sur la protection de la vie privée, quelques formulaires secondaires ont été enlevés de cette thèse.

Bien que ces formulaires aient inclus dans la pagination, il n'y aura aucun contenu manquant. 


\begin{abstract}
Recent computer attacks target networks and there is a need to detect these network anomalies quickly and efficiently. This area has been widely studied and solutions typically use data not freely available. A labeled available dataset, Kyoto2006+, has been recently created. Most existing works using Kyoto2006+ apply various clustering approaches. Our research leverages existing spectral analysis and statistical analysis techniques.

One well known spectral analysis technique is Haar Wavelet filtering analysis. It measures the amount and magnitude of abrupt changes in data. Another popular approach is a statistical analysis technique, Principle Component Analysis (PCA). PCA describes data in a new dimension to better express the data. A modified PCA which incorporates time shifting to account for changes over time is considered. Both approaches have strengths and limitations. In response, this thesis proposes Hybrid PCA - Haar Wavelet Analysis. This approach uses PCA to describe the data and Haar Wavelet filtering for analysis.

Based on prototyping and measurement, an investigation of the Hybrid PCA - Haar Wavelet Analysis technique is performed. Experimental results are presented to demonstrate the accuracy and precision of the combined approach compared to the two algorithms individually. Furthermore, tests to examine the impact of algorithm parameters are discussed.
\end{abstract}




\section{Acknowledgements}

I would like to express my sincere thanks to my thesis supervisors, Professor Chung-Horng Lung and Professor Ioannis Lambadaris, for all their guidance, and support throughout the completion of this thesis. Furthermore, I would like to thank the entire SCE office staff and SCE technical support for all their help. In addition, I would like to thank Nic Falvo and the Graduate Student Association for all their support. I am grateful to Carleton University, Ontario Center for Excellence (O.C.E.) and the Government of Ontario for providing financial support for this research. I would also like to acknowledge Solana Networks for their financial support and collaboration in this research.

Last but not least, I would like to thank a handful of very special people who supported me these last several semesters. Without their kind words or few moments of care, I could not have accomplished my work: Mike Floyd, Angad Gakhar, James Green, Norman Lim, Andre Loiselle, Allana Krolak-Lee, Hannan Mahmoud, Ziyad Muslat, Quynh-Chi Nguyen, Simon Skowronski, Mario Valiente, Patrick Visinski, Tina Woods and my most of all, my mother, Anna Buraczewski. I am blessed to have such strong and good people in my life. 


\section{Table of Contents}

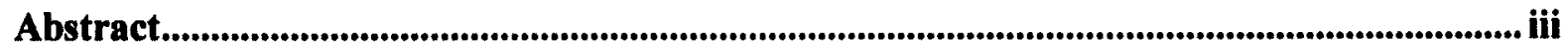

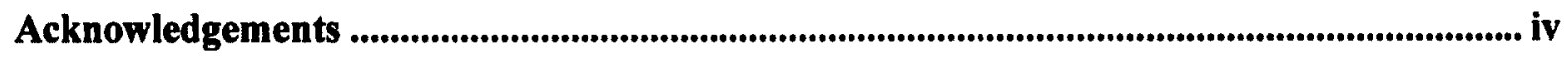

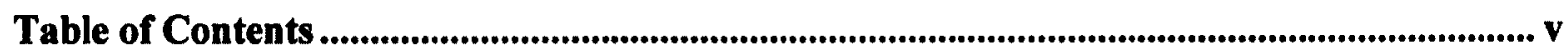

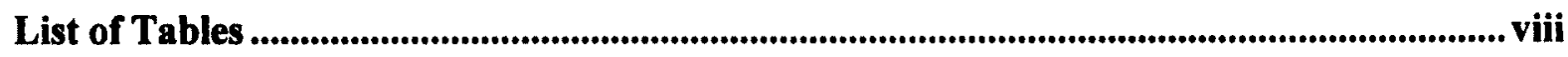

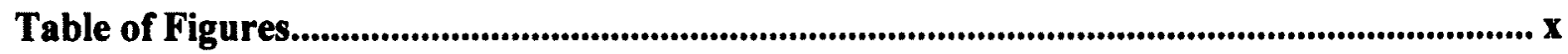

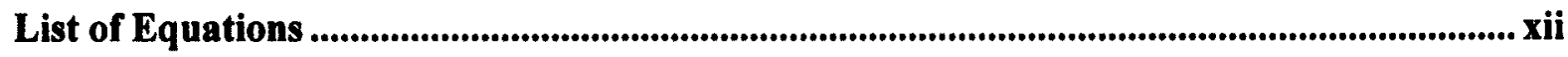

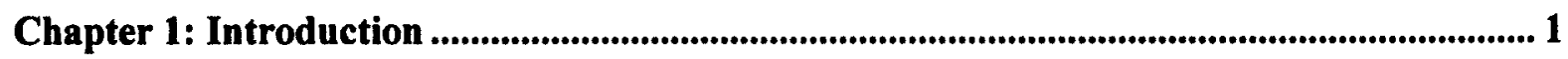

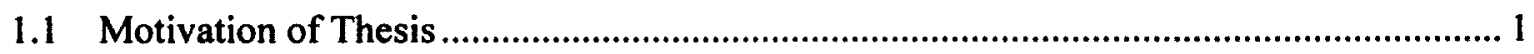

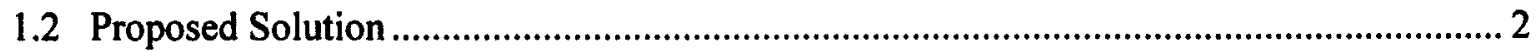

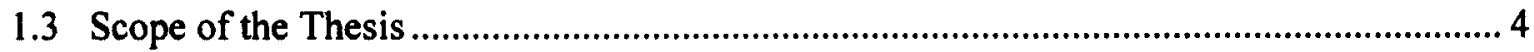

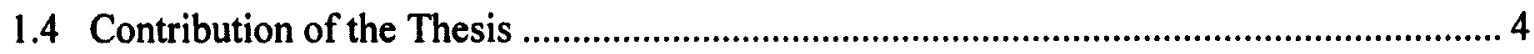

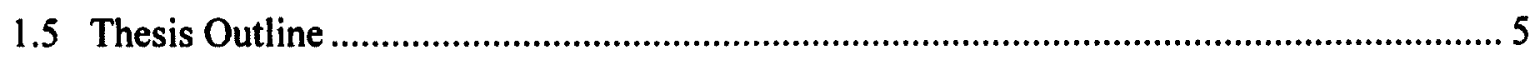

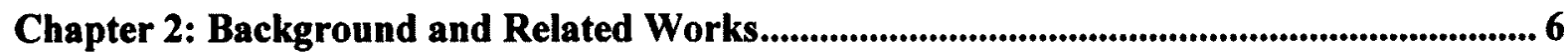

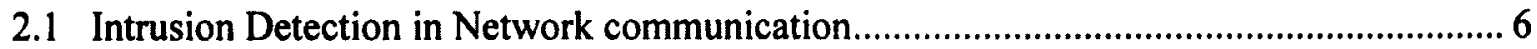

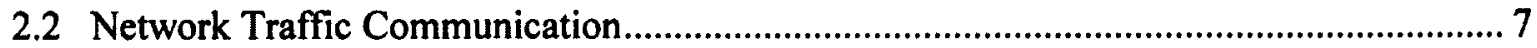

2.2.1 Network Traffic Data Sources...................................................................... 8

2.2.2 Network Traffic Volume .......................................................................... 10

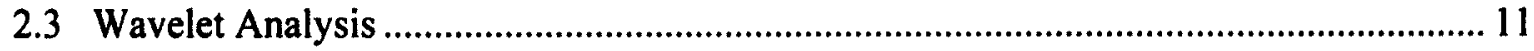

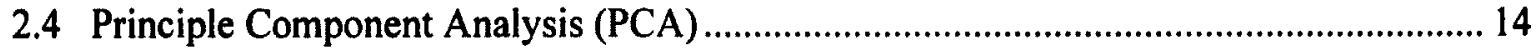

2.5 Comparison with Related Works ............................................................................ 17

Chapter 3: Anomaly Detection Data, Algorithms, and Threshold Techniques .................... 21

3.1 Network Traffic Data and Data Profiles ................................................................. 21

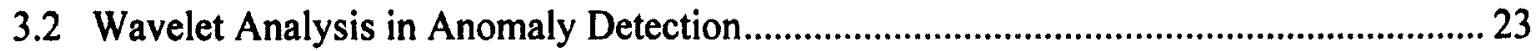

3.3 Modified Principle Component Analysis in Anomaly Detection .................................... 26

3.4 A Hybrid Analysis in Anomaly Detection.................................................................. 29

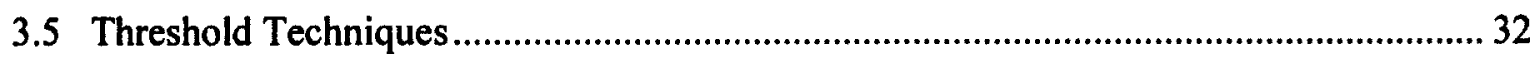

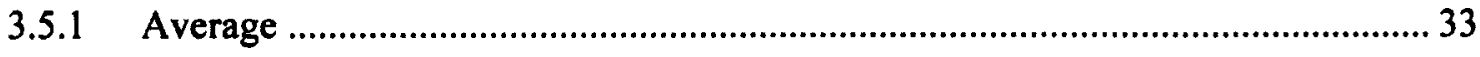

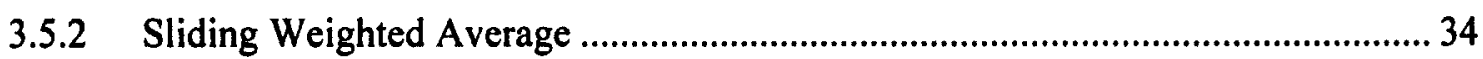


3.5.3 Average without outliers .............................................................................. 34

Chapter 4: Prototype Design and Implementation............................................................................... 36

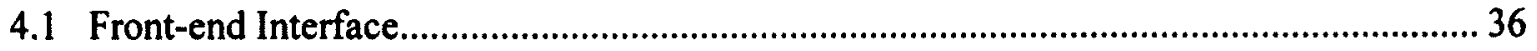

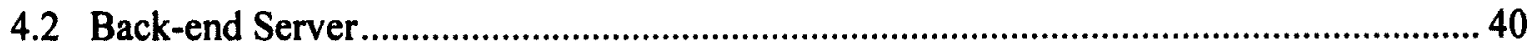

Chapter 5: Experiments and Evaluation ................................................................................................... 42

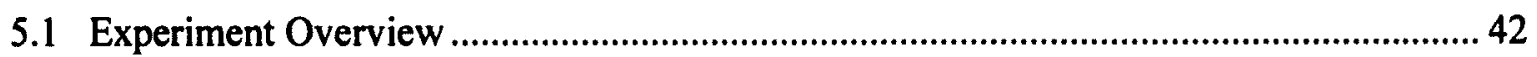

5.2 Experimental Setup ................................................................................................. 42

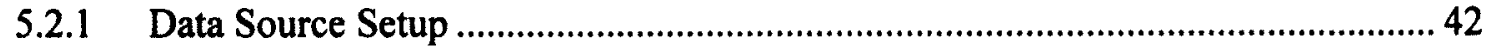

5.2.2 Performance Measurement Setup .......................................................................... 46

5.2.2.1 Hit and Miss Count................................................................................................ 46

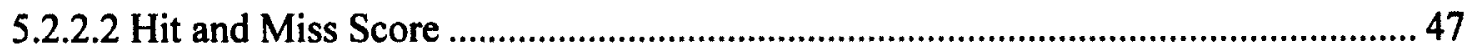

5.3 Experimental Procedure ............................................................................................ 48

5.3.1 Test Set Creation ............................................................................................. 49

5.3.2 Modified Principle Component Analysis Experiments............................................. 49

5.3.3 Haar Wavelet Filtering Analysis Experiments ....................................................... 51

5.3.4 Hybrid PCA - Haar Wavelet Filtering Analysis Experiments ............................... 52

Chapter 6: Experimental Results and Discussion................................................................................. 53

6.1 Evaluation of Modified Principle Component Analysis ..................................................... 53

6.1.1 Modified PCA and Pure Data...........................................................................5

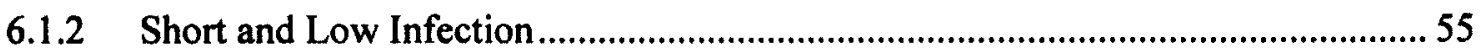

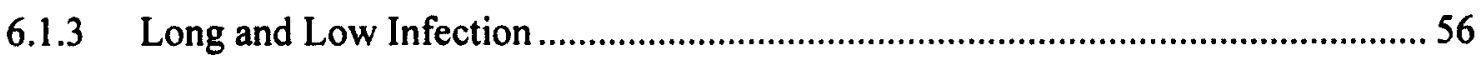

6.1.4 Short and High Infection ................................................................................. 57

6.1.5 Long and High Infection ................................................................................ 58

6.1.6 Varying number of Time Shifts......................................................................... 59

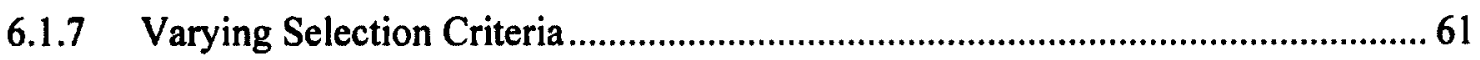

6.1.8 Threshold Techniques and Modified PCA ........................................................... 63

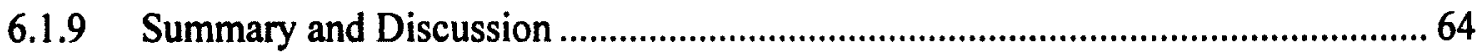

6.2 Evaluation of Haar Wavelet Analysis........................................................................6 66

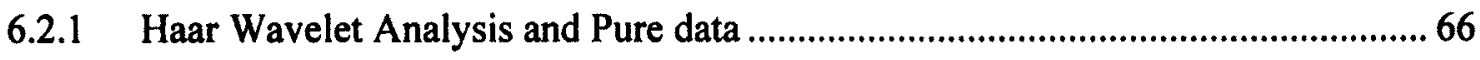

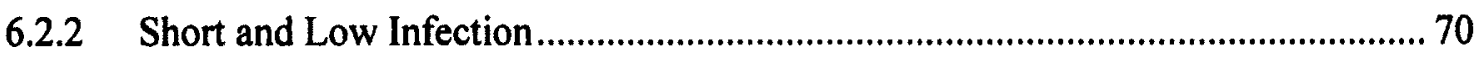




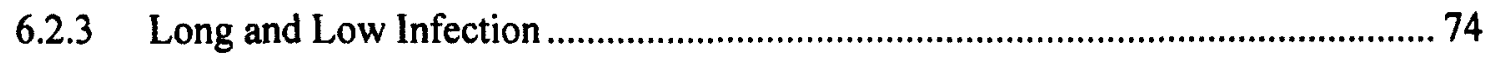

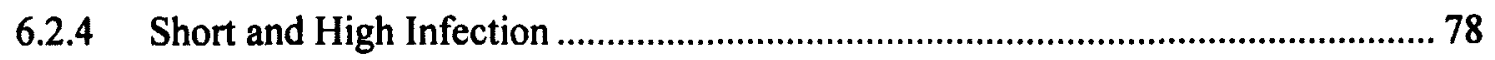

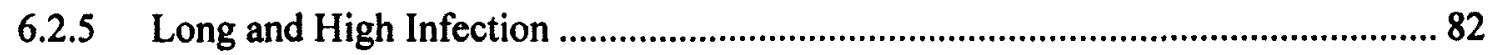

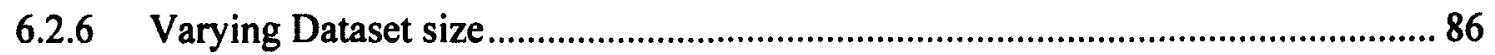

6.2.7 Varying Local Variability Weights .................................................................... 93

6.2.8 Threshold Techniques and Haar Wavelet Filtering analysis.............................. 102

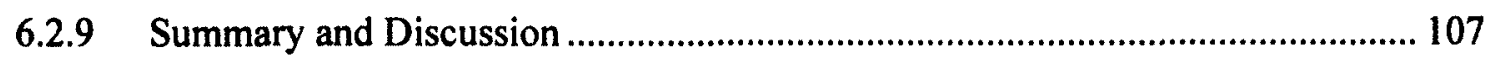

6.3 Evaluation of Hybrid PCA - Haar Wavelet Filtering Analysis ...................................... 108

6.3.1 Hybrid Analysis and Pure data ................................................................... 108

6.3.2 Short and Low Infection .............................................................................. 110

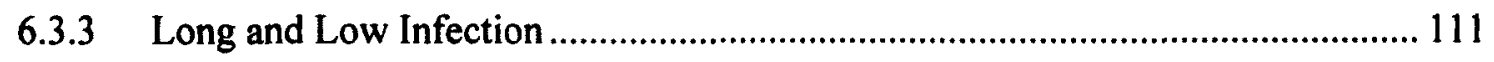

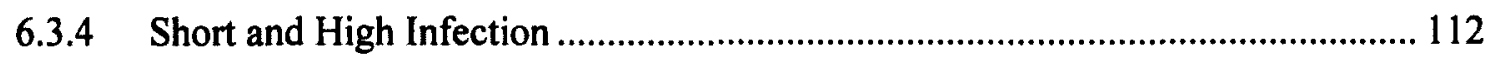

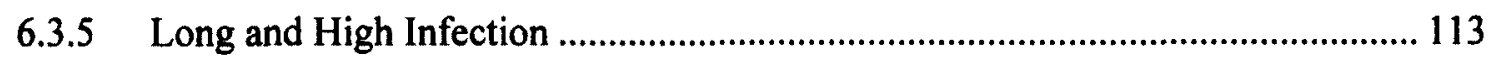

6.3.6 Threshold Techniques and Hybrid PCA - Haar Wavelet filtering Analysis........ 115

6.3.7 Summary and Discussion ............................................................................. 117

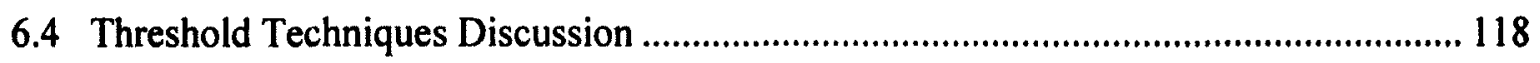

Chapter 7: Conclusion .............................................................................................................. 119

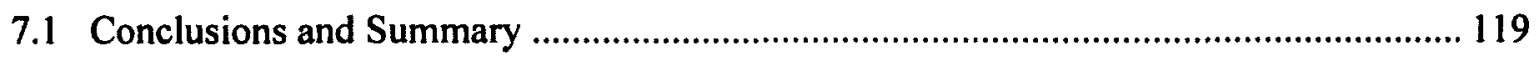

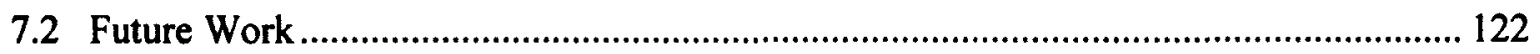

References........................................................................................................................................................... 124 


\section{List of Tables}

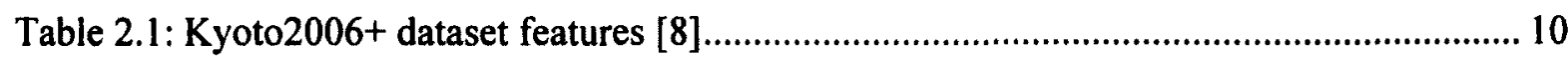

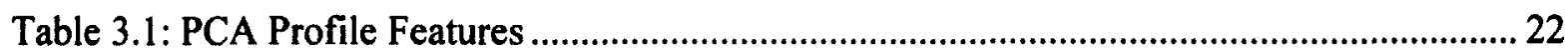

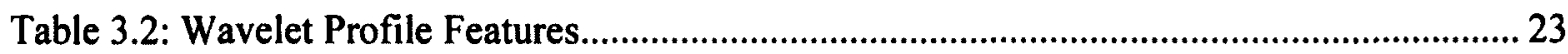

Table 5.1: Kyoto2006+ flow count breakdown per month .......................................................... 44

Table 5.2: Dates in experimentation by scenario and analysis duration .................................. 45

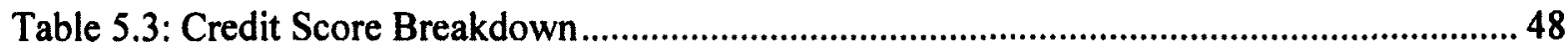

Table 5.4: Outline of parameters used in experimentation .................................................... 49

Table 6.1: March 8, 2009 - PCA experiment results ............................................................5 55

Table 6.2: May 28, 2009 - PCA experiment results ............................................................. 57

Table 6.3: January 15, 2009 - PCA experiment results ............................................................ 58

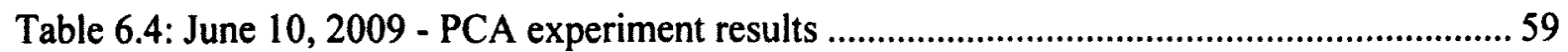

Table 6.5: March 8, 2009 - PCA Short and Low infection varying time shift results..................60

Table 6.6: May 28, 2009 - PCA Long and Low infection varying time shift results .................. 60

Table 6.7: January 15, 2009 - PCA Short and High infection varying time shift results ............. 60

Table 6.8: June 10, 2009 - PCA Long and High infection varying time shift results.................. 61

Table 6.9: March 8, 2009 - PCA Short and Low infection varying selection criteria results..... 61

Table 6.10: May 28, 2009 - PCA Long and Low infection varying selection criteria results ..... 62

Table 6.11: January 15, 2009-PCA Short and High infection varying selection criteria results 62

Table 6.12: June 10, 2009 - PCA Long and High infection varying selection criteria results.... 62

Table 6.13: March, 8, 2009 - PCA Short and Low threshold results...........................................63

Table 6.14: May 28, 2009 - PCA Long and Low threshold results .......................................... 63

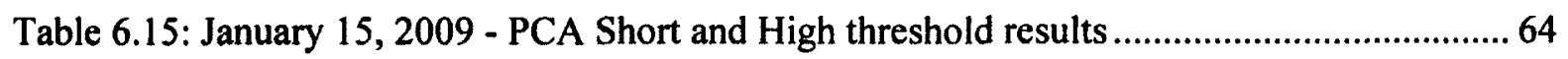

Table 6.16: June 10,2009 - PCA Long and High threshold results .......................................... 64

Table 6.17: March 8, 2009 - Wavelet Experiment results ........................................................ 73

Table 6.18: May 28, 2009 - Wavelet Experiment results ............................................................ 77

Table 6.19: January 15, 2009 - Wavelet Experiment results ................................................. 81

Table 6.20: June 10, 2009 - Wavelet Experiment results .................................................... 85

Table 6.21: March 8, 2009 Varying input data / boundary size results .................................... 87 
Table 6.22: May 28, 2009 Varying input data / boundary size results ..................................... 89

Table 6.23: January 15, 2009 Varying input data / boundary size results ................................ 91

Table 6.24: June 10, 2009 Varying input data / boundary size results ....................................... 93

Table 6.25: March 8, 2009 Varying Local Variability weights results ..................................... 95

Table 6.26: May 28, 2009 Varying Local Variability weights results..................................... 97

Table 6.27: January 15, 2009 Varying Local Variability weights results .................................99

Table 6.28: June 10, 2009 - Varying Local Variability weights results .................................. 101

Table 6.29: March 8, 2009 - Wavelet Short and Low threshold results ................................ 103

Table 6.30: May 28, 2009 - Wavelet Long and Low threshold results .................................. 104

Table 6.31: January 15, 2009 - Wavelet Short and High threshold results ............................. 105

Table 6.32: June 10, 2009 - Wavelet Long and High threshold results.................................. 106

Table 6.33: March 8, 2009 - Hybrid experiment results ..................................................... 111

Table 6.34: May 28, 2009 - Hybrid experiment results..................................................... 112

Table 6.35: January 15, 2009 - Hybrid experiment results................................................... 113

Table 6.36: June 10, 2009 - Hybrid experiment results......................................................... 114

Table 6.37: March 8, 2009 - Hybrid threshold results............................................................. 115

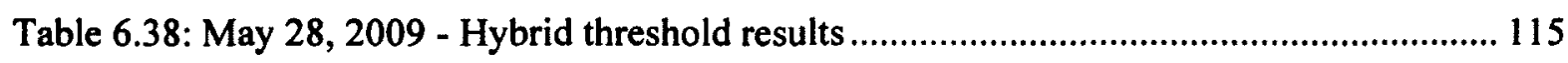

Table 6.39: January 15, 2009 - Hybrid threshold results.................................................. 116

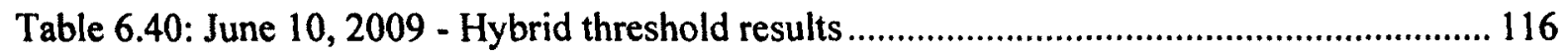

Table 6.41: Summary of differences between algorithms ................................................. 117 


\section{Table of Figures}

Figure 2.1: Creating a flow in the NetFlow cache [2]

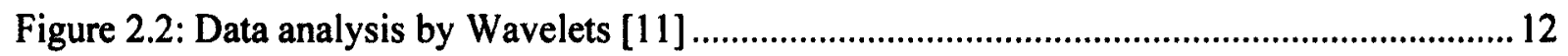

Figure 2.3: Haar Wavelet decomposition ................................................................................. 14

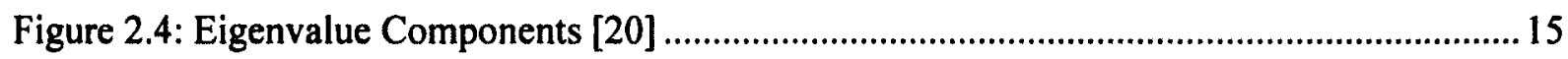

Figure 3.1: Activity Diagram of Haar Wavelet Filtering Approach ..................................................25

Figure 3.2: Local Variability score for March 8, 2009 with 5\% infection rate ................................26

Figure 3.3: Time shifting Illustration .........................................................................................2

Figure 3.4: Activity Diagram of Modified PCA approach ........................................................29

Figure 3.5: SPE score of March 8, 2009 for 5\% infection rate......................................................29

Figure 3.6: Hybrid PCA-Haar Wavelet Filtering activity diagram................................................. 32

Figure 3.7: Delta score for March 8, 2009 for $5 \%$ infection rate........................................................ 32

Figure 4.1: Test bed system diagram ............................................................................................ 36

Figure 4.2: Use case diagram of test bed system .......................................................................... 38

Figure 4.3: Screen capture of SPE scores plotted from test bed ........................................................39

Figure 4.4: Screen capture of information panel from test bed ...................................................... 40

Figure 4.5: Class diagram of backend server from test bed............................................................. 41

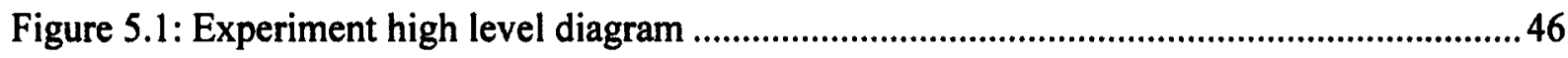

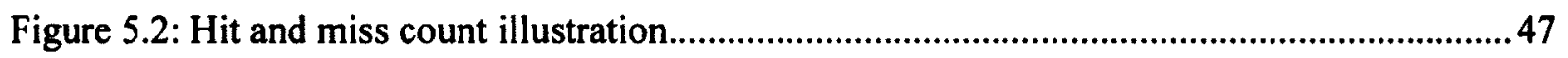

Figure 6.1: SPE scores from Unfiltered Modified PCA analysis on January 6, 2009 data ............54

Figure 6.2: Subset of SPE scores from Modified PCA analysis on January 6, 2009 data...............54

Figure 6.3: SPE scores from Filtered Modified PCA analysis on January 6, 2009 data ..................55

Figure 6.4: March 8, 2009 - PCA SPE scores 5\% (top left), 10\% (top right), 20\% (bottom) results 56

Figure 6.5: May 28, 2009 - PCA SPE scores 5\% (top left), 10\% (top right), 20\% (bottom) results 57

Figure 6.6: January 15, 2009 - PCA SPE scores $75 \%$ (top left), 100\% (top right), 120\% (bottom) results .58

Figure 6.7: June 10, 2009 - PCA SPE scores 75\% (top left), 100\% (top right), 120\% (bottom) results 59

Figure 6.8: January 6, 2009 - OTHER Wavelet flows Local Variability Scores. .67 
Figure 6.9: January 6, 2009 - TCP Wavelet flows Local Variability Scores................................68

Figure 6.10: January 6, 2009 - Average OTHER Wavelet flows Local Variability Scores ...........69

Figure 6.11: January 6, 2009 - Average TCP Wavelet flows Local Variability Scores ................. 70

Figure 6.12: March 8, 2009 - Average OTHER Wavelet Local Variability Scores with 5\%

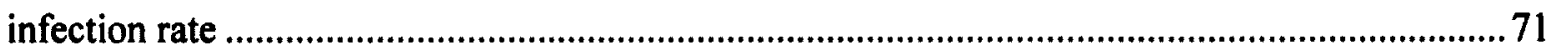

Figure 6.13: March 8, 2009 - Average TCP Wavelet Local Variability Scores with 5\% infection

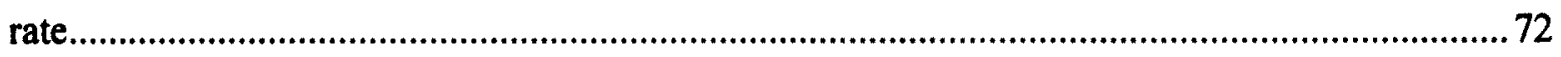

Figure 6.14: May 28, 2009 - Average OTHER Wavelet Local Variability Scores with 5\%

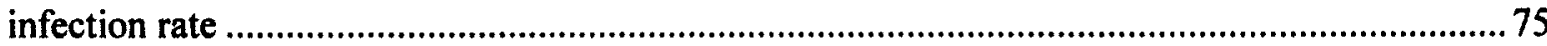

Figure 6.15: May 28, 2009 - Average TCP Wavelet Local Variability Scores with 5\% infection

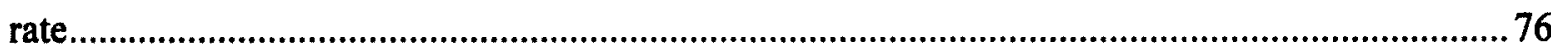

Figure 6.16: January 15, 2009 - Average OTHER Wavelet Local Variability Scores with 5\% infection rate .79

Figure 6.17: January 15, 2009 - Average TCP Wavelet Local Variability Scores with 5\% infection rate 80

Figure 6.18: June 10, 2009 - Average OTHER Wavelet Local Variability Scores with 5\% infection rate .83

Figure 6.19: June 10, 2009 - Average TCP Wavelet Local Variability Scores with 5\% infection rate. 84

Figure 6.20: January 6, 2009 - Hybrid Delta scores (top) and Average Delta scores (bottom)... 109 Figure 6.21: March 8, 2009 - Hybrid Delta Scores of 5\% (top left), 10\% (top right), 20\%(bottom) infection rates 110

Figure 6.22: May 28, 2009 - Hybrid Delta Scores of 5\% (top left), 10\% (top right), 20\% (bottom) infection rates

Figure 6.23:January 15, 2009 - Hybrid Delta Scores of 75\% (top left), 100\% (top right), $120 \%$ (bottom) infection rates

Figure 6.24:June 10, 2009 - Hybrid Delta Scores of 75\% (top left), 100\% (top right), 120\% (bottom) infection rates. 114 


\section{List of Equations}

Equation 2.1: Haar Wavelet basis function [11] ................................................................ 12

Equation 2.2: Haar Wavelet scaling function [11]................................................................... 13

Equation 2.3: Haar Wavelet decomposition function [11] .................................................... 13

Equation 2.4: Haar Wavelet frequency adjusted basis function [11]......................................... 13

Equation 2.5: Principle Component Formula [16].................................................................... 15

Equation 2.6: Matrix of covariance estimates [16] ................................................................ 16

Equation 3.1: Normalization Formula .............................................................................. 25

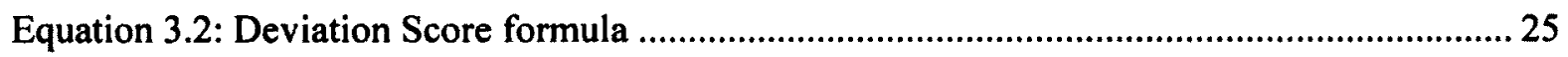

Equation 3.3: Local Variability formula .................................................................................... 25

Equation 3.4: Normal Space formula [16] ............................................................................. 28

Equation 3.5: Abnormal Space formula [16] ........................................................................... 28

Equation 3.6: Square Prediction Error (SPE) formula [16] ................................................... 28

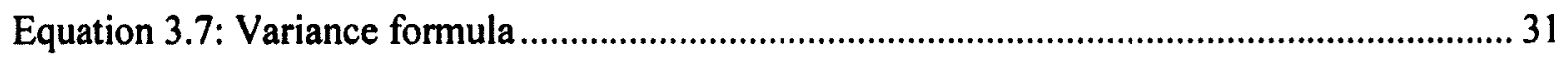

Equation 3.8: Delta score formula .......................................................................................... 31

Equation 3.9: Static Average formula........................................................................................ 33

Equation 3.10: Moving Average formula ................................................................................. 33

Equation 3.11: Weighted Moving Average formula................................................................. 34

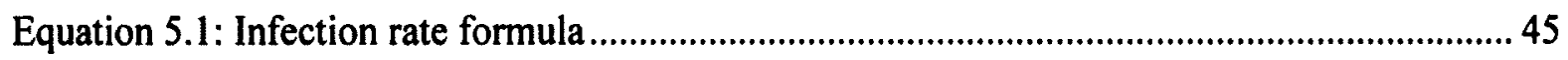




\subsection{Introduction}

The way networks are being used is rapidly changing and a by-product of this change is the amount of network traffic volume is rapidly increasing. Similarly, the types of computer attacks are rapidly evolving. For example, malicious attacks are no longer limited to desktop computer viruses, but can target a network itself. These attacks are designed to create failures in the system. A failure brings upon anomalous network behaviour and can be categorized into two forms, soft failures and hard failures. A soft failure can be thought of as a performance degradation; whereas, a hard failure would be elements ceasing to operate [1]. Depending on the network, these failures can cause mild inconveniences, loss of productivity, loss of economic activity, or even, loss of public well being. This research applies statistical analysis and spectral analysis techniques to network traffic data in order to identify potential malicious network attacks. Specifically, this thesis focuses on a hybrid technique to provide information to a network operator such that the source of malicious behaviour can be isolated.

\subsection{Motivation of Thesis}

There is a need for effective and scalable approaches to maintain network stability and to detect anomalous network traffic behaviour created by attacks. The state of the art to manage massive amounts of data transmission is Cisco's NetFlow protocol [2]. This protocol resides on routers and each packet that passes through is examined for a set of IP packet attributes. The output of NetFlow is a multi-tuple record, called a flow. Some core features of a flow are: Source IP address, Destination IP address, total bytes, etc (See Figure 2.1 for an illustration of NetFlow collection). NetFlow does not provide any information if the flow is a part of abnormal or malicious behaviour [3]. 
Current intrusion detection systems (IDS), such as Bro, use existing knowledge base and identify attacks through patterns for signatures [4]. As a result of the high volume of traffic, inspecting individual signatures or traces of known hazards is time consuming and inefficient. Furthermore, the turnaround from discovery to updating the knowledge base can be extensive. Another approach, called anomaly detection, attempts to detect patterns which do not conform to expected behaviour [5]. Anomaly detection does not have to be dependent on an existing knowledge base. Some statistical models and signal processing algorithms do not require an existing knowledge base. These methods can be applied relatively quickly to create relationships and discover patterns from a range of data types and sizes. The types of computer attacks rapidly change, and hackers are constantly coming up with new techniques. As a result, no matter how refined the detection method becomes; a comprehensive IDS will require a significant amount of human expertise [5].

\subsection{Proposed Solution}

This thesis proposes a hybrid solution, based on statistical and spectral analysis techniques, which provide the network administrator time slices of potential network traffic intrusions. To the best of our knowledge, no such hybrid techniques has deployed by systems described in existing literature.

The statistical analysis studied is a Modified Principal Component Analysis (PCA) technique to determine abnormal behaviour [6]. Components are extracted by comparing feature data similarity. For example, consider a two-dimensional data set, a line of best fit can be considered as a component (See Figure 2.4). A ranked subset of components, selected by comparing sparsity of projected data, is used to create a subspace that describes anomalous 
behaviour. Time grouped data is projected onto this space and spectral analysis is applied. The Modified PCA algorithm is described in more detail in section 3.1.1. The feature with the most spread out data is considered in spectral analysis portion.

The spectral analysis technique adopted is Haar Wavelet decomposition [7]. This type of wavelet decomposition uses a Haar basis function to decompose the input dataset set into core time functions. Thresholds are applied to remove noise and highlight network traffic anomaly characteristics. The signal is reconstructed and a weighted score to describe the magnitude of fluctuations within each time slice is calculated. The Haar wavelet algorithm is described in more detail in section 3.1.2. A high score represents a large change in a time window and suggests to the network administrator that abnormal and potentially malicious behaviour is present.

This research uses a hybrid technique and illustrates how much more accurate and informative it is compared to the statistical and spectral analysis techniques independently. This hybrid approach examines network traffic data grouped into time bins and summarized. Next, it applies statistical analysis to reduce the complex nature of the network traffic. It then applies spectral analysis to determine time slices of interest where there is a change in behaviour. Network traffic data can be grouped by start time into bins and features describing each time bin behaviour are extracted (See Table 3.1.2 and Table 3.1.3 for a complete outline of features). The time binning approach is described in more detail in section 3.1 .

To evaluate the performance of the algorithms, a prototype test bed is used. Furthermore, a novel labeled dataset, called Kyoto2006+, was used in analysis [8]. The design and implementation of the test bed is discussed in chapter 4 and an outline of the dataset is presented in Section 2.2.1. 


\subsection{Scope of the Thesis}

This thesis focuses on the hybrid approach which is based on a modified PCA approach and a Haar wavelet approach. As discussed in Section 1.2, network traffic data is grouped into time bins, and descriptive features are derived. Then a hybrid analysis approach is applied is obtain a measure of potential anomalies for each time slice. The algorithm can provide a time slice, and features of interest as breadcrumbs, to lead to the offending source. As no specific host is highlighted, manual investigation of the network traffic data is required. A subsequent technique applied on the time slice to isolate the core source of the anomalous behaviour is beyond the scope of this thesis and can form a direction for future research. Further discussion is provided in Section 7.2 .

\subsection{Contribution of the Thesis}

The main contributions of the thesis are presented next.

- A hybrid analysis: a novel technique which uses a statistical analysis technique, Principle Component Analysis, to reduce the complexity of the time binned dataset and produces an anomalous subspace. Next, a spectral analysis technique, Haar wavelet decomposition, is applied to highlight network traffic anomalies and to produce a measure of potential malicious behaviour.

- Independent applications of statistical analysis and spectral analysis applied on Kyoto2006+ dataset. The Kyoto2006+ dataset is a network traffic dataset which provides intrusion information and anti-virus alerts from existing open source detectors. To the best of our knowledge, none of the works using the Kyoto2006+ dataset have focused on Haar wavelet decomposition or Principle Component Analysis.

- Investigation of several algorithms for performance measurement and studying the impact in order to automatically indicate a set time slices which may contain potentially malicious behaviour. 
- A prototype test bed is built and the performance of each of the algorithms is evaluated through various measurements made by the system. A number of insights into the algorithm behaviour and performance are obtained from the experimental results that include the following:

- A demonstration of the effectiveness of each of the adopted approaches for identifying network anomalies is presented. The performance improvement of the hybrid approach compared to only the statistical approach and only the spectral approach is discussed.

- The effect of different algorithm parameter values on the performance of the approaches is presented.

\subsection{Thesis Outline}

The rest of the thesis is organized as follows. Chapter 2 provides background information on network traffic and anomaly detection, and discusses related works. Chapter 3 outlines the statistical analysis algorithm, the spectral analysis algorithm and the hybrid algorithm in more detail. Chapter 4 describes the test bed prototype design and operation. Chapter 5 outlines each step of the experimental approach in detail. Chapter 6 presents and discusses the results of the performance analysis on the hybrid approach, on only the statistical approach and on only the spectral approach. Finally, Chapter 7 concludes the thesis and discusses possible directions for future research. 


\subsection{Background and Related Works}

This chapter presents background information on network traffic. It then describes spectral and statistical analysis techniques in intrusion detection. In addition, it provides a background about the algorithms used in this research. Lastly, the chapter discusses related works.

\subsection{Intrusion Detection in Network communication}

Network traffic intrusion detection is identification of observed activities that do not conform to an established norm in computer communication [9]. There exist two main types of intrusion detection methods: misuse detection and anomaly detection. Misuse detection is based on an existing knowledge of known attacks and identifies attacks through patterns or signatures [9]. As a result of the high volume of traffic, inspecting individual packets for signatures or traces of known hazards is time consuming and inefficient. Anomaly detection is not dependent on an existing knowledge base and identifies potential network threats by finding deviations from normal behaviour.

Spectral analysis, also known as frequency analysis, looks at time-varying signals to reveal special characteristic [10]. Wavelet analysis is one form of spectral analysis and divides a complicated function into several simple ones. The term wavelet comes from the characteristic of converging to zero, and is illustrated by waving above and below the horizontal axis [11]. It represents a signal by a finite sum of components of different resolutions. Individual components can be examined for anomalies [12]. Statistical analysis deals with the discrimination, classification and interpretation of collected datasets [13]. In this research, principle component analysis is considered. PCA aims to represent data in a lower dimensional 
space through a transformation of the original features. The new variables or features are uncorrelated orthogonal linear combinations of the original dataset. They are a ranked in order of significance and describe trends in data with greater details [13]. Anomaly detection techniques are applied to these components. These various anomaly detection techniques can be applied relatively quickly to create relationships between observed variables over a range of data types and determine deviations.

\subsection{Network Traffic Communication}

Traditional computer network communication use a specific protocol to route data from an origin to a destination. A packet is a unit of data which contains user application information and control information used to properly deliver the data. A network protocol is a set of standards used to format communication between source and destinations [14]. A widely used protocol is Transmission Control Protocol (TCP). A session or flow is a set of packets sent from the same source and source port to a specific destination and destination port with the same network protocol. The network traffic software collects the data for the flow until the flow is terminated. It can be terminated with either a timeout or a finish flag, FIN. [2]

A flow can be described by a number of characteristics or features and are measured by software on network routers or network probes [3]. These probes examine the control information of data packets. An example is Cisco's NetFlow, an industry standard protocol, which describes a flow through seven features: source IP address, destination IP address, source port, destination port, protocol type, Class of Service, and Router or switch interface [2]. The observation software is loaded on Cisco routers (See Figure 2.1). 


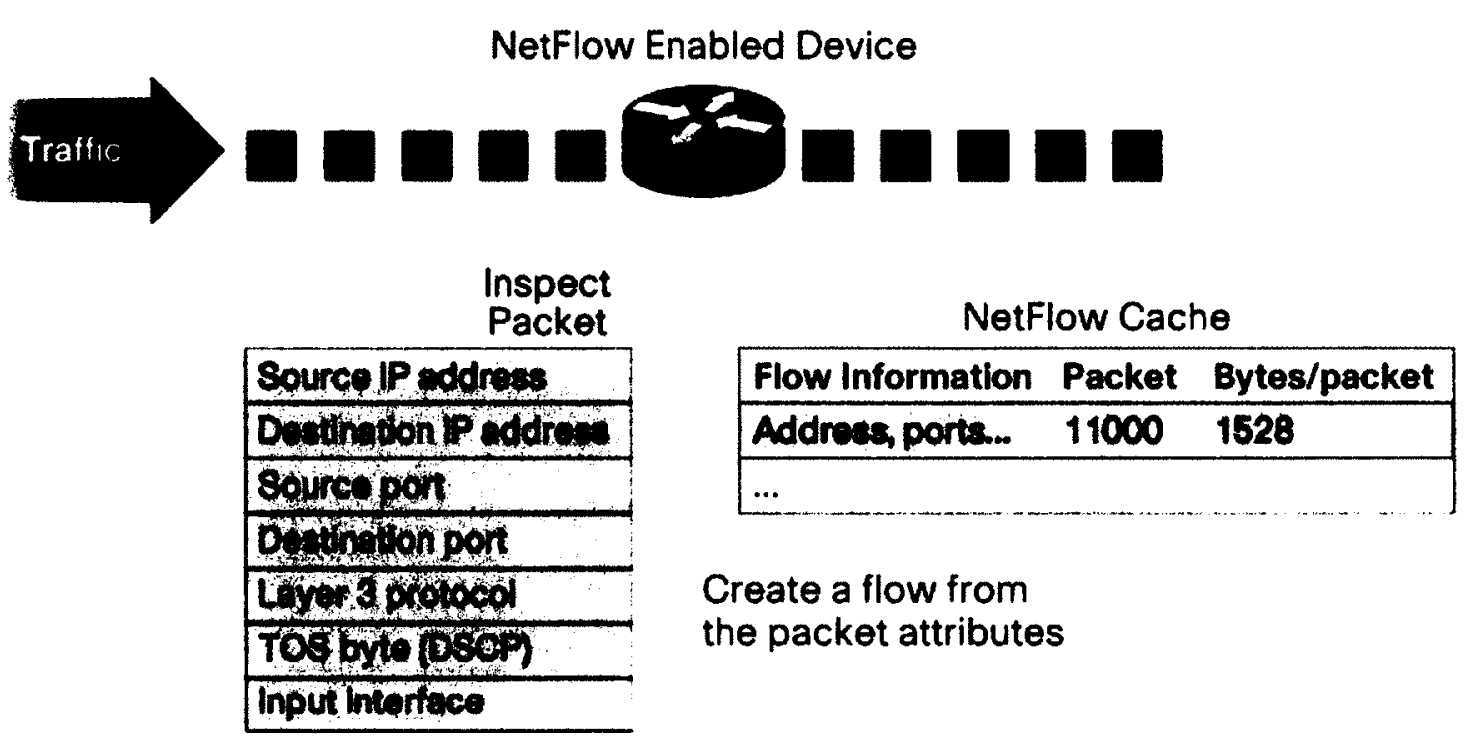

Figure 2.1: Creating a flow in the NetFlow cache [2]

\subsubsection{Network Traffic Data Sources}

A number of the data sources in anomaly detection research are from large research networks. An example of this is the Geant research network based in Europe [15]. This network connects over 30 European countries and connects national and education computer networks [15]. Although there are many research networks which create datasets, it has become extremely difficult to obtain data due to privacy concerns and competitive issues.

In 1999, a competition was held at the Knowledge Discovery and Data Mining annual conference to build a network intrusion detection system that would detect anomalies. Network traffic data was made up of nine weeks of raw TCP dump data. The data simulated network traffic on a typical U.S. Air Force LAN and takes about $743 \mathrm{MB}$ of memory. The nine weeks were broken up into seven weeks of training, and two weeks of testing data. Unlike many research networks, which only provide flow data or sanitized raw TCP data, each observation contains anomaly labels and descriptions. This data source has been widely used in research. While it is very commonly used in algorithm evaluation; there are two major drawbacks. The 
data is more than ten years old and does not reflect current threats [8]. Even though the there are 41 features describing a flow, the most common descriptors, such as source port, are omitted. This is a major drawback as many popular approaches, like in [16] and [6], use various entropy measurements for their analysis. [17]

In response to this major drawback, Song et al. [8] created a new evaluation dataset called Kyoto2006+. This novel dataset was used in this research. It contains over three years of real traffic data and described by 24 characteristics or features. The primary 14 statistical features are derived from the KDD'99 dataset. The authors analyzed the features of this dataset and kept only those they believed relevant. Then the authors added six features of connection information, which was previously omitted. An example is source address, source port, destination address, etc. The remaining four features provide detector information that, to the best of our knowledge, no other research dataset provides. The authors used open source tools; an intrusion detection software tool called SNORT, an anti virus program called ClamAV and the Ashula detection software to detect exploit code. Along with the output information from these programs, the authors provide a "label" feature is a cross correlation between their research findings and that of the open source tools. (See Table 2.1 for an outline of the features). [8]

\begin{tabular}{|c|l|}
\hline Feature Name & \multicolumn{1}{|c|}{ Description } \\
\hline Duration & The length (seconds) of the connection. \\
\hline Service & The connection's service type, e.g., http, telnet. \\
\hline Source bytes & The number of data bytes sent by the source IP address. \\
\hline Destination bytes & The number of data bytes sent by the destination IP address \\
\hline Count & $\begin{array}{l}\text { The number of connections whose source IP address and destination } \\
\text { IP address are the same to those of the current connection in the past } \\
\text { two seconds. }\end{array}$ \\
\hline Same srv rate & Percentage of connections to the same service in Count feature \\
\hline Serror rate & Percentage of connections that have "SYN" errors in Count feature \\
\hline Srv serror rate & $\begin{array}{l}\text { Percentage of connections that have "SYN" errors in Srv count(the } \\
\text { number of connections whose service type is the same to that of the } \\
\text { current connection in the past two seconds) feature }\end{array}$ \\
\hline
\end{tabular}




\begin{tabular}{|c|c|}
\hline Dst host count & $\begin{array}{l}\text { Among the past } 100 \text { connections whose destination IP address is the } \\
\text { same to that of the current connection, the number of connections } \\
\text { whose source IP address is also the same to that of the current } \\
\text { connection. }\end{array}$ \\
\hline Dst host srv count & $\begin{array}{l}\text { Among the past } 100 \text { connections whose destination IP address is the } \\
\text { same to that of the current connection, the number of connections } \\
\text { whose service type is also the same to that of the current connection }\end{array}$ \\
\hline $\begin{array}{l}\text { Dst host same src } \\
\text { port rate }\end{array}$ & $\begin{array}{l}\text { Percentage of connections whose source port is the same to that of the } \\
\text { current connection in Dst host count feature }\end{array}$ \\
\hline Dst host serror rate & $\begin{array}{l}\text { Percentage of connections that have "SYN" errors in Dst host count } \\
\text { feature }\end{array}$ \\
\hline $\begin{array}{l}\text { Dst host srv serror } \\
\text { rate }\end{array}$ & $\begin{array}{l}\text { Percentage of connections that "SYN" errors in Dst host srv count } \\
\text { feature }\end{array}$ \\
\hline Flag & The state of the connection at the time the connection was written. \\
\hline IDS detection & $\begin{array}{l}\text { Reflects if IDS triggered an alert for the connection; ' } 0 \text { ' means any } \\
\text { alerts were not triggered, and an arabic numeral means the different } \\
\text { kinds of the alerts. Parenthesis indicates the number of the same alert. }\end{array}$ \\
\hline Malware detection & $\begin{array}{l}\text { Indicates if malware, also known as malicious software, was } \\
\text { observed in the connection; '0' means no malware was observed, and } \\
\text { a string indicates the corresponding malware observed at the } \\
\text { connection. Parenthesis indicates the number of the same malware. }\end{array}$ \\
\hline Ashula c & $\begin{array}{l}\text { Means if shellcodes and exploit codes were used in the connection; } \\
\text { ' } 0 \text { ' means no shellcode nor } 35 \text { exploit code was observed, and an } \\
\text { arabic numeral means the different kinds of the shellcodes or exploit } \\
\text { codes. Parenthesis indicates the number of the same shellcode or } \\
\text { exploit code. }\end{array}$ \\
\hline Label & $\begin{array}{l}\text { Indicates whether the session was attack or not; ' } 1 \text { ' means the session } \\
\text { was normal, '-1' means known attack was observed in the session, } \\
\text { and ' }-2 \text { ' means unknown attack was observed in the session. }\end{array}$ \\
\hline Source IP Address & $\begin{array}{l}\text { Means the source IP address used in the session. The original IP } \\
\text { address on IPv4 was sanitized. }\end{array}$ \\
\hline Source Port Number & Indicates the source port number used in the session. \\
\hline $\begin{array}{l}\text { Destination IP } \\
\text { Address }\end{array}$ & It was also sanitized. \\
\hline $\begin{array}{l}\text { Destination Port } \\
\text { Number }\end{array}$ & Indicates the destination port number used in the session. \\
\hline Start Time & Indicates when the session was started. \\
\hline Duration & Indicates how long the session was be \\
\hline
\end{tabular}

Table 2.1: Kyoto2006+ dataset features [8]

\subsubsection{Network Traffic Volume}

The volume of network traffic data is very high and there is need for efficient and scalable solutions for network anomaly detection. Considering traffic data alone can be 
excessive, and overly granular. Depending on the data source, raw traffic data can consist of millions of observations. One approach to limit data size, which would not add any extra overhead, is to create smaller summaries or profiles. A profile consists of composite features, such as summations, or statistical features, such as entropy. The most common approach, as seen in [16] and [6], is to create a number of time slices and assigning the flows, by start time, to each time slice. Then, a set of features describe each of these time bins. For example, in [6], the TCP traffic in a time slice was summarized by seven features: total bytes, total packets, total number of flows, destination address entropy, unique number of hosts, and unique number of destinations. Alternatively, Wanner [18] modeled each observation around a node. Each unique source node in the dataset was described through 41 composite features. These features consisted of total bytes received by the host, total bytes sent by the host, total number of unique

ports, etc. Both approaches produce an observation table much smaller than the primary flow data set. In this research, time bin profiling is used. The number of observations comes down from millions of flows to a few thousand hosts, or a few hundred time bins.

\subsection{Wavelet Analysis}

Recently wavelets have become popular due to the effectiveness of representing time varying signals in a compact fashion. Unlike Fourier transforms, which use the sine and cosine function as bases, wavelets use signals localized in time and frequency [10]. Wavelet decomposition provides accurate resolution both the time and frequency domain [19]. Wavelets can represent a signal by a finite sum of component functions [12]. The components can be examined for anomalies (See Figure 2.2 for block diagram of wavelet data analysis). A natural extension of collecting metrics is to organize the observations by time. The input time signal is 
first decomposed using wavelet decomposition into several orthogonal components. Next, threshold techniques are applied. According to a set of parameters and depending on the type of analysis, various values are filtered out or set to zero. Lastly, the signal is reconstructed to produce a filtered time signal, where parameter dependant behaviour is illustrated. [11]

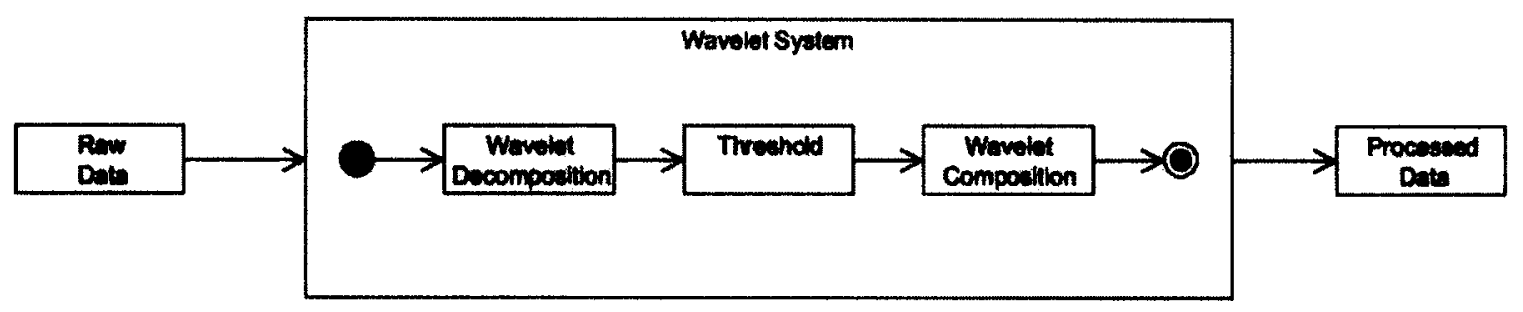

Figure 2.2: Data analysis by Wavelets [11]

There are different types of wavelet approaches, and each has their own basis function and decomposition approaches. The simplest wavelet analysis, used in this research, is the Haar wavelet analysis [10]. The Haar wavelet basis function, $\psi(x)$ (See Equation 2.1), and the scaling function, $\phi(x)$ (See Equation 2.2), are used to create a piecewise decomposition of the input time signal. The decomposition function is a sum of the scaling function and basis functions. (See Equation 2.3 for the decomposition function). The scaling function is used to make the decomposition discrete, such that an infinite amount of pieces are not needed to represent the input signal. In the decomposition, versions of the Haar function, denoted $\psi_{j k}(x)$ (See Equation 2.4), are translated and dilated to represent different frequency resolutions, specified by $j$ and $k$. These components are orthonormal which means that they are independent, non-overlapping and analysis produces distinct results. As shown in [11], in order satisfy that the frequency components are orthonormal, the const from equation 2.4 must equal $2^{\mathrm{j} / 2}$.

$$
\psi(x)=\left\{\begin{array}{cl}
1 & 0 \leq x<1 / 2 \\
-1 & 1 / 2 \leq x<1
\end{array}\right.
$$

Equation 2.1: Haar Wavelet basis function [11] 


$$
\phi(x)=10 \leq x \leq 1
$$

Equation 2.2: Haar Wavelet scaling function [11]

$$
f(x)=C_{00}(x)+\sum_{j=0}^{n-1} \sum_{k=0}^{2 j_{-1}} D_{j k} \psi_{j k}(x)
$$

Equation 2.3: Haar Wavelet decomposition function [11]

$$
\psi_{j k}(x)=\text { const } * \psi\left(2^{j} x-k\right)
$$

Equation 2.4: Haar Wavelet frequency adjusted basis function [11]

The input signal size is directly proportional to the complexity of the decomposition function. The larger the input signal, the more coefficients are produced. Usually one can assign a frequency range to each component. Each coefficient scales a specific component. Haar wavelet filtering applies threshold techniques to these coefficients. Depending on the type of filter, a number of coefficients are assigned to zero. Typical filtering approaches include high pass frequency filters, low pass frequency filters and medium frequency filters. The user determines the filtering by providing a specific frequency range. Filtering occurs through the coefficients outside this range being set to zero. After filtering is applied, the signal is then reconstructed by reversing the decomposition approach. The result is a filtered version of the original signal.

The Haar Wavelet Filtering approach bisects the input data between higher and lower frequency values given a certain input. The lower pass frequency values are then fed back into the system until a defined limit is reached. A visualization of Equation 2.3 can be seen in Figure 2.3. Each iteration is referred to as a level and has its own set of components which correspond to a certain frequency range. It is possible to describe filters or frequency ranges by the number of levels applied. For example, in Figure 2.3, a dataset of eight observations is filtered. The four 
high pass values can be considered to be in level one, the two mid pass values can be considered in level two and the two remaining are low pass in level three. In order to apply filtering, the component coefficients for a specific level are set to zero. For example, in Figure 2.3, a high pass filter would set the coefficients at levels two and three to zero, and then reconstruct the signal.

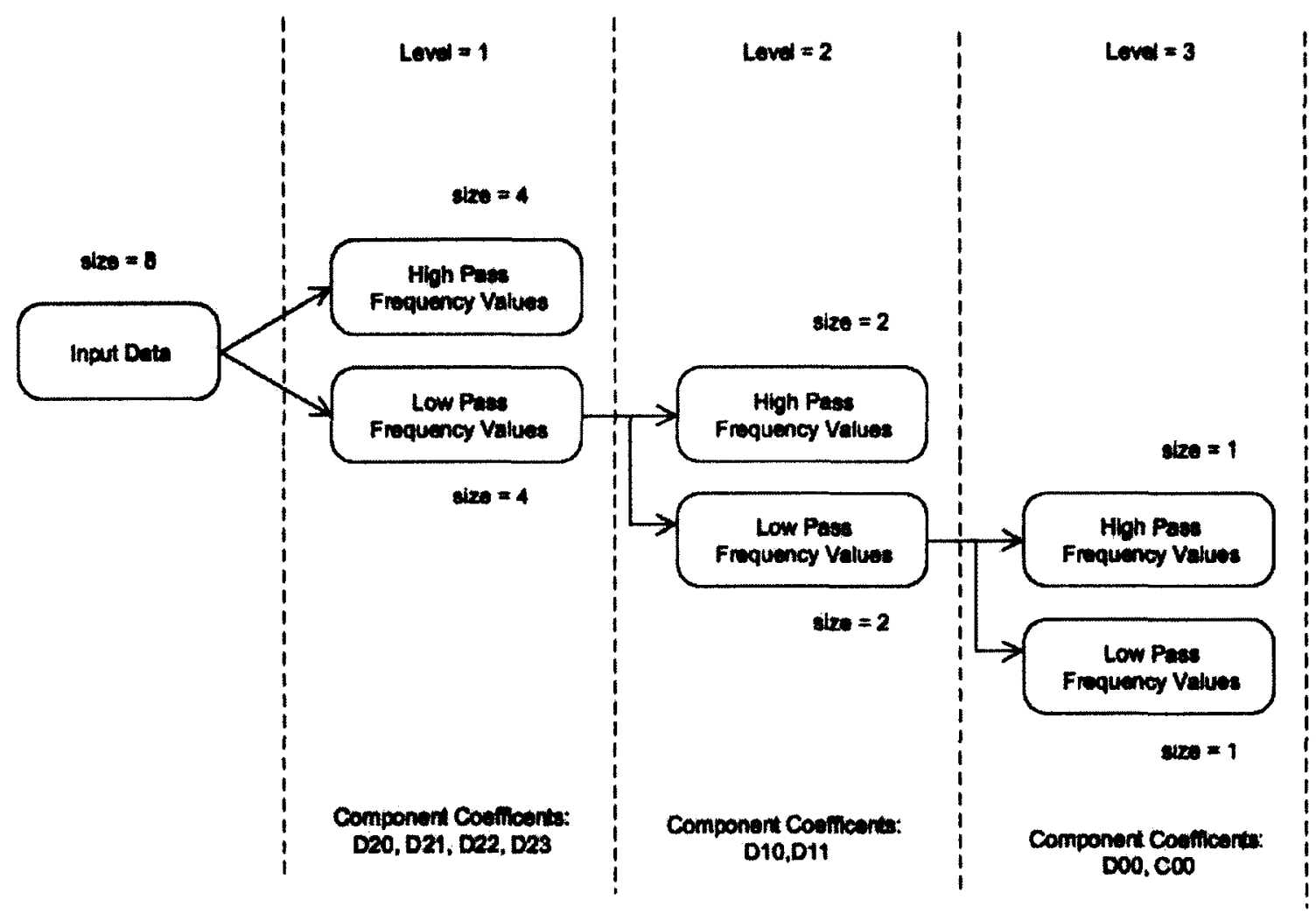

Figure 2.3: Haar Wavelet decomposition

\subsection{Principle Component Analysis (PCA)}

Principle Component Analysis derives new variables or features that are linear combinations of the original features and are uncorrelated [13]. It is a means of finding a reduced set of features which describe the dataset by examining the relationships between features. PCA is a transformation that can be considered as a rotation of the original axes to a 
new set of orthogonal axes. It can be used as feature selection, as it determines a new set ranked component. For example, in a two-dimensional dataset, a line of best fit on a Cartesian Graph would be considered a principle component (See Figure 2.4 for an illustration) [20].

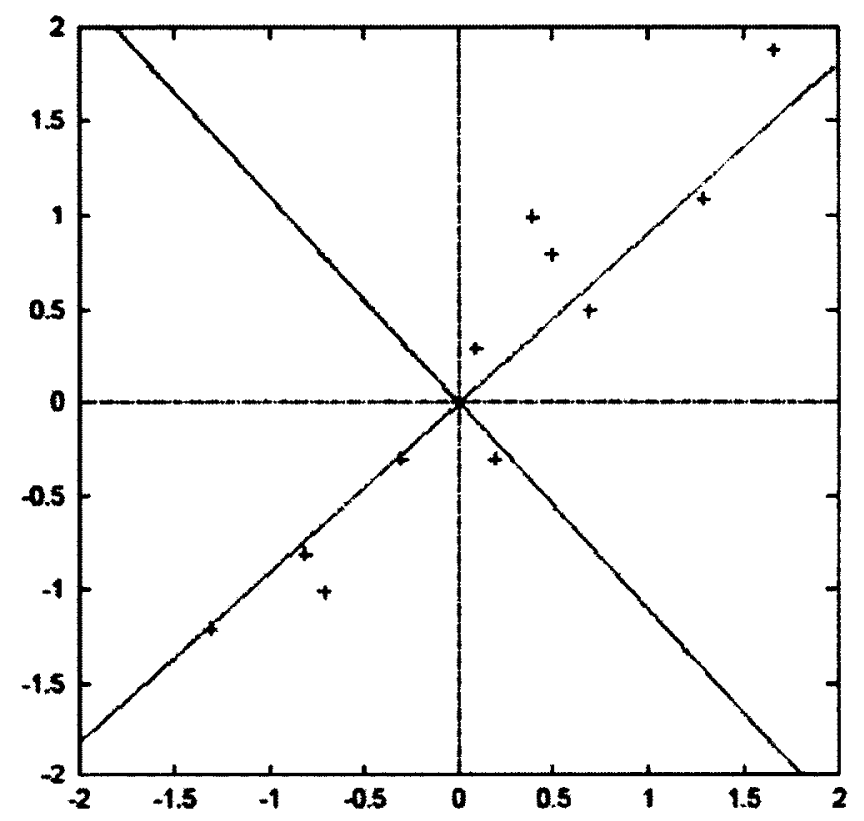

Figure 2.4: Eigenvalue Components [20]

There are a number of methods in deriving the set of principle components, $\xi$. In this research, eigenvalue decomposition approach is used. Typically, the new components will not have a zero mean. Therefore, the data must be standardized in order to create a zero mean. This requires that each original features set are shifted to have a zero mean. The principle components can be obtained by using Equation 2.5, where $\mathrm{A}$ is a matrix of observation coefficients, $\mathrm{x}$ is a matrix of random vectors, $\mathrm{x}_{1} . . \mathrm{x}_{\mathrm{p}}$, or features, and $\mathrm{u}$ the sample mean.

$$
\xi=A^{T}(x-\mu)
$$

Equation 2.5: Principle Component Formula [16] 
As shown in [20], using eigenvector decomposition to determine the principle components, $\xi$, requires calculating the covariance matrix of $(x-\mu)$, as illustrated in equation 2.6. A covariance matrix is produced to illustrate the variance, or measure the spread of data, between every component combination (See Equation 2.6). The eigenvectors of $\Sigma$ are the principle components. The nature of eigenvector decomposition produces the eigenvector and eigenvalues to be ordered from greatest to least. The eigenvalues are the measure of variance when the dataset get projected onto its respective eigenvector [21]. As a result, the list of eigenvectors is a ranked from most to least significant. These eigenvectors are the set of axis that characterize the data. Considering the previous example of two-dimensional data, an eigenvector would be the actual line of best fit.

$$
\text { Covariance Matrix }=\frac{1}{n-N-1} x^{T} x
$$

Equation 2.6: Matrix of covariance estimates [16]

There are many components determined from decomposition. The number of components can equal the number of initial features. The components are ranked from most to least significant. The principle components are based on the type of selection criteria applied. The most basic selection criterion is 'Top $\mathrm{K}$ ' components. The first $K$ many elements are considered as the principle components. Another approach is based on the distribution of the data projected onto each of the components. Variance is the square root of standard deviation, and a standard deviation of one suggests that the dataset is normally distributed. This approach considers any eigenvector a principle component if its corresponding eigenvalue is greater than one. Lastly, an approach considered is to build a space which covers $99 \%$ of the data. The space starts with the most significant principle component. One by one, components are added to the 
space until the sum of the variances in the space is equal to or greater than $99 \%$ of the sum of all the variances. The type of selection criteria used directly affects the number of principle components extracted.

\subsection{Comparison with Related Works}

Intrusions Detection Systems are widely used in practice, as well as a subject of research. They are seen as one of the most promising countermeasures to defend computer systems and networks against attackers [22]. Existing systems detect intrusions by examining and comparing raw traffic with an existing knowledge base. Although theses have systems have contributed a great deal to the network traffic security domain, a number of weaknesses still exist [23]. Open source IDS, such as Snort, monitor network traffic to detect invalid communication using predefined attacks [24]. Anomaly detection methods can examine large amounts of data in a real time. Machine learning techniques require less expert knowledge and provide good performance; however, a truly comprehensive IDS will always require human expertise [5]. A number of systems were examined from literature and most proposed multi step approaches. The most recent approach, proposed by Zheng et al. [25], described a multi step approach based on Support Vector Machines(SVM) on time binned NetFlow data. After identifying malicious time bins, the authors' used Kullback-Leibler distance between the test set and training set to identify the features contributing to the anomaly [25]. Another approach, proposed in [22], involved data collection, conversion into session data and feature extraction. Training data was used to build a normal profile and anomaly detection was conducted using a variety of clustering algorithms. In [26] a similar approach was adopted; however, clustering was used to filter data and Support Vector Machine algorithms were used to detect anomalies. In [27], a multi step 
detector using wavelets was proposed. Adaptive threshold methods were first applied for quick detection and then finer analysis was applied using continuous wavelet transforms. In [28], a five step approach was proposed. It involves data conversion, feature analysis, wavelet transform, statistical analysis and threshold techniques, wavelet synthesis and anomaly detection. The types of anomaly detection methods in anomaly detection systems vary in literature; but, to the best of our knowledge, all propose multi-step approaches.

The first steps consistently involved data collection and data conversion. None of the machine learning techniques reviewed analyze raw network traffic data. There are various methods to characterize the data for analysis. As seen in [29], raw TCPDUMP data from 1999 DARPA ID dataset is converted into network flow logs which based on different basic metrics. In Barford et al. [7], analysis is only applied to byte and packet counts, over five minute sampling intervals, from wide-area routing links. Although [17] does not reflect current network situations; it is still commonly used in research [8]. Another method used in literature is NetFlow and it is the industry standard to collect and characterize network traffic data [2]. In Lakhina et al. [16], NetFlow data was grouped into a time series and projected onto an anomalous subspace. In [30], the time bin features used in [16] were used and discussed how easily they can be scaled to larger and more diverse datasets. Brauckhoff et al. [6] used three weeks of NetFlow data from August 2007 by aggregating the traffic at 15 minute intervals. Alternatively, [31] show that there exists different ways of time binning data which offer good performance for tracking per-flow data. It presented an alternative to NetFlow.

It is common for Intrusion Detection Systems to consider raw network traffic that has been characterized different ways, such as NetFlow. The data is further profiled in order to reduce data volume and detection algorithms are applied. These detection methods can be 
performed either real-time or offline [5]. Alarms can be generated through inspecting the results of the algorithm and is required for automated detection. For example, if a database is used, SQL triggers which initiate a predefined action for a specific condition can be used [5]. Typically, threshold techniques are used to anomalies automatically. Yet, considering the importance of the network administrator, visual plotting of the output is beneficial.

In this research, Principle Component Analysis was used. It has been used in pervious works, such as [16], [30], [6] and [15]. Lakhina et al. [16] first made this approach popular. Ringberg et al. [15] examined [16] and critiqued it. Through extensive analysis, they showed that selection criterion to build the anomalous subspace directly impacts the error rate. More specifically, the PCA algorithm is very sensitive to top $\mathrm{k}$ parameter, and it varies across different networks or aggregations [15]. The authors found that sufficiently large anomalies can contaminate the operation of the algorithm. Lastly, they observed that the PCA algorithm has different effectiveness under different traffic types and measurements. In [15], it was concluded that it is difficult to pinpoint exact anomalous flows. Brauckhoff et al. [6] also examined the works proposed by [16], and suggested that the standard PCA analysis lacked temporal consideration. They suggested implementing a time shift technique in order to compensate for data trends over time.

This research also looked at spectral analysis and wavelet analysis. There are different variations of wavelet analysis, and there have been different works on it. In [27], the continuous wavelet transform was proposed to detect Denial of Service attacks. Other works were based on the Discrete Wavelet Transform. As outlined in [32], an approach which uniquely applied four algorithms which were based on different basis functions. In [7], an approach used to identify frequency characteristics of anomalous network traffic methods. It focused on identifying 
anomalies by removing predictable, ambient parts and only then employing statistical methods [7].

There have been few works comparing the spectral and statistical analysis techniques in the same way our research does. One hybrid approach, as outlined in [33], proposed a multi-step approach that used a Kalman filter to filter out normal traffic and wavelet filtering to process the residual data. The authors concluded that their approach did not perform very well. There has been some work done comparing the statistical and spectral analysis approach used in our work. In [19], the authors examined the PCA and wavelet anomaly detection methods. The authors observed that wavelets are just as good as entropy-PCA in most cases. Considering a Distributed Denial of Service attacks, it was found wavelets performed better. The authors observed that the effectiveness of entropy-PCA depends strong on top $k$ parameter [19]. The authors performed extensive analysis and concluded that there exists no method consistently better than the other in detecting all types of anomalies. There were scenarios where both methods were inconclusive. 


\subsection{Anomaly Detection Data, Algorithms, and Threshold}

\section{Techniques}

This chapter describes profiling approaches applied to the network traffic data. The three anomaly detection algorithms are described: the Haar wavelet decomposition algorithm, the modified principle component analysis algorithm and the hybrid analysis algorithm. These algorithms are applied to profiled network traffic data in order to get a measure of anomalous behaviour. Lastly, the threshold techniques used to automatically isolate potential anomalous time slices are discussed.

\subsection{Network Traffic Data and Data Profiles}

The network traffic dataset used in this research is the Kyoto2006+ dataset [8]. This dataset provides session information, anti-virus and intrusion detector information and sourcedestination routing information (See Table 2.1 for a complete listing of features). Different data profiles were created to reduce the traffic volume. One type of profile was for wavelet analysis, and the other for PCA analysis. The hybrid approach used profiles created for PCA analysis. Each profile describes a certain period of time or time bin, and no time bins overlap. Each of these time bins have a start time, end time and various aggregate features derived from flows which fall within that particular time bin. For example, total bytes is the total amount of bytes transmitted by all flows with a start time after the bin start time and before the bin end time (See Table 3.1 and Table 3.2 for a complete listing of features for the wavelet and PCA profiles). The anomaly detection algorithms were applied to these profiles in order to determine potential time slices with malicious behaviour. 


\begin{tabular}{|c|c|}
\hline Features & Description \\
\hline Start_time & $\begin{array}{l}\text { The beginning of the time bin. All flows } \\
\text { beginning by this time and ending by the } \\
\text { corresponding end time are placed into the } \\
\text { particular bin. }\end{array}$ \\
\hline End_time & $\begin{array}{l}\text { The finish of the time bin. All flows } \\
\text { beginning by the corresponding start time } \\
\text { and ending by this time are placed into the } \\
\text { particular bin. }\end{array}$ \\
\hline TCP_Bytes & $\begin{array}{l}\text { Total number of bytes of the TCP protocol } \\
\text { in this particular time bin. }\end{array}$ \\
\hline TCP_Flows & $\begin{array}{l}\text { Total number of flows of the TCP protocol } \\
\text { in this particular time bin. }\end{array}$ \\
\hline TCP_Source_IP_Address_Entropy & $\begin{array}{l}\text { Entropy of source IP addresses from TCP } \\
\text { protocol flows in this particular time bin. }\end{array}$ \\
\hline TCP_Destination_IP_Address_Entropy & $\begin{array}{l}\text { Entropy of destination IP addresses from } \\
\text { TCP protocol flows in this particular time } \\
\text { bin. }\end{array}$ \\
\hline TCP_Unique_Source_IP_Address_Count & $\begin{array}{l}\text { Total number distinct source IP addresses } \\
\text { from TCP protocol flows in this particular } \\
\text { time bin. }\end{array}$ \\
\hline TCP_Unique_Destination_IP_Address_Count & $\begin{array}{l}\text { Total number distinct destination IP } \\
\text { addresses from TCP protocol flows in this } \\
\text { particular time bin. }\end{array}$ \\
\hline OTHER_Bytes & $\begin{array}{l}\text { Total number of bytes not of the TCP } \\
\text { protocol in this particular time bin. }\end{array}$ \\
\hline OTHER_Flows & $\begin{array}{l}\text { Total number of flows not of the TCP } \\
\text { protocol in this particular time bin. }\end{array}$ \\
\hline OTHER_Source_IP_Address_Entropy & $\begin{array}{l}\text { Entropy of source IP addresses not from } \\
\text { TCP protocol flows in this particular time } \\
\text { bin. }\end{array}$ \\
\hline OTHER_Destination_IP_Address_Entropy & $\begin{array}{l}\text { Entropy of destination IP addresses from } \\
\text { TCP protocol flows in this particular time } \\
\text { bin. }\end{array}$ \\
\hline OTHER_Unique_Source_IP_Address_Count & $\begin{array}{l}\text { Total number distinct source IP addresses } \\
\text { not from TCP protocol flows in this } \\
\text { particular time bin. }\end{array}$ \\
\hline OTHER_Unique_Destination_IP_Address_Count & $\begin{array}{l}\text { Total number distinct destination IP } \\
\text { addresses not from TCP protocol flows in } \\
\text { this particular time bin. }\end{array}$ \\
\hline
\end{tabular}

Table 3.1: PCA Profile Features 


\begin{tabular}{|l|l|}
\hline \multicolumn{1}{|c|}{ Features } & \multicolumn{1}{c|}{ Description } \\
\hline Start_time & $\begin{array}{l}\text { The beginning of the time bin. All flows beginning } \\
\text { by this time and ending by the corresponding end } \\
\text { time are placed into the particular bin. }\end{array}$ \\
\hline End_time & $\begin{array}{l}\text { The finish of the time bin. All flows beginning by } \\
\text { the corresponding start time and ending by this } \\
\text { time are placed into the particular bin. }\end{array}$ \\
\hline TCP_Bytes & $\begin{array}{l}\text { Total number of bytes of the TCP protocol in this } \\
\text { particular time bin. }\end{array}$ \\
\hline TCP_Flows & $\begin{array}{l}\text { Total number of flows of the TCP protocol in this } \\
\text { particular time bin. }\end{array}$ \\
\hline TCP_Source_IP_Address_Entropy & $\begin{array}{l}\text { Entropy of source IP addresses from TCP protocol } \\
\text { flows in this particular time bin. }\end{array}$ \\
\hline TCP_Destination_IP_Address_Entropy & $\begin{array}{l}\text { Entropy of destination IP addresses from TCP } \\
\text { protocol flows in this particular time bin. }\end{array}$ \\
\hline TCP_Source_Port_Entropy & $\begin{array}{l}\text { Entropy of source ports from TCP protocol flows } \\
\text { in this particular time bin. }\end{array}$ \\
\hline TCP_Destination_Port_Entropy & $\begin{array}{l}\text { Entropy of destination ports from TCP protocol } \\
\text { flows in this particular time bin. }\end{array}$ \\
\hline OTHER_Bytes & $\begin{array}{l}\text { Total number of bytes not of the TCP protocol in } \\
\text { this particular time bin. }\end{array}$ \\
\hline OTHER_Flows & $\begin{array}{l}\text { Total number of flows not of the TCP protocol in } \\
\text { this particular time bin. }\end{array}$ \\
\hline OTHER_Source_IP_Address_Entropy & $\begin{array}{l}\text { Entropy of source IP addresses not from TCP } \\
\text { protocol flows in this particular time bin. }\end{array}$ \\
\hline OTHER_Destination_IP_Address_Entropy & $\begin{array}{l}\text { Entropy of destination IP addresses from TCP } \\
\text { protocol flows in this particular time bin. }\end{array}$ \\
\hline OTHER_Source_Port_Entropy & $\begin{array}{l}\text { Entropy of source ports not from TCP protocol } \\
\text { flows in this particular time bin. }\end{array}$ \\
\hline OTHER_Destination_Port_Entropy & $\begin{array}{l}\text { Entropy of destination ports not from TCP protocol } \\
\text { flows in this particular time bin. }\end{array}$ \\
\hline & Wriprofles
\end{tabular}

Table 3.2: Wavelet Profile Features

\subsection{Wavelet Analysis in Anomaly Detection}

Wavelet analysis examined how quickly data changed and the magnitude of this change over time. The wavelet transformations reveal the composition of a signal in terms of the building blocks, or translated basis functions, of the transformed domain [10]. The decomposition resulted in a set of orthogonal signals or components which combined, describe the signal entirely. In our application, the input into the system was the wavelet time bin profiles 
(See Table 3.2 for an outline of the input features). The wavelet transformation applied in this thesis was the Haar wavelet decomposition. Haar wavelets are the most basic wavelets and are able to approximate any continuous function [10]. The Haar wavelet analysis can only examine single dimensional signals. In this research, the input signal was each of the feature observations. Each of the features from Table 3.2 were applied independently as input signals. Each was decomposed and frequency components of the signal were examined in an attempt to detect patterns which could be previously missed.

The process of applying Haar wavelet decomposition to network anomaly detection consists of three main steps. First, a Haar wavelet high pass filter, low pass filter and mid pass filter were each independently applied to the input data (See Figure 3.1 for a filtering diagram). As outlined in [7] the motivation for frequency filtering in anomaly detection was that the high frequency values highlight the start and end times of the anomalous behaviours and the mid pass frequency values highlight the duration of the potential attacks. Next, each of the filtered datasets was normalized by using Equation 3.1. The local variability over a sliding window was calculated on each of the normalized datasets. The Haar wavelet filtering occasionally produced an output of negative values which is not semantically meaningful in the network traffic domain. The local variability provided a positive measurement of how sparse the data in the window was (See Equation 3.2). Specifically, a larger local variability indicates a greater change of values in the window. Lastly, in order to obtain a single scalar value in time, the deviation score was calculated. The deviation score was a summation of weighted local variability values for each point in time. This deviation score was a measure of how often and how much values change at a point in time (See Equation 3.3). Either a plot of these scores was produced for visual 
inspection, or threshold techniques were applied for automated classification of anomalous time bins (See Figure 3.2).

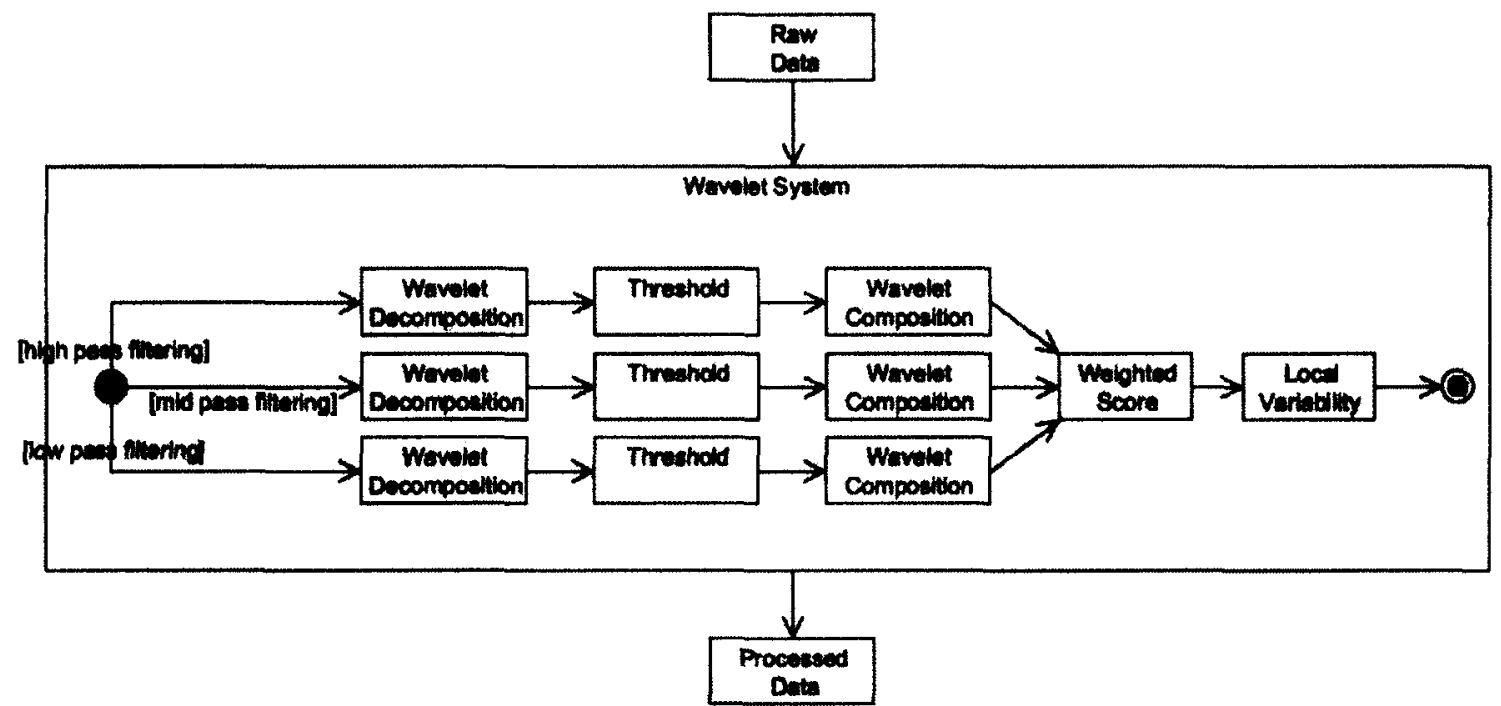

Figure 3.1: Activity Diagram of Haar Wavelet Filtering Approach

$$
\text { Normalized }_{i}=\frac{o b s_{i}-\text { mean(obs) }}{\text { standardDeviation(obs) }}
$$

Normalizedi - normalized observation element $\mathrm{i}$ obs - set of observations

Equation 3.1: Normalization Formula

\section{DeviationScore $_{i}(H, M, L)=H \times$ highpass $_{i}+M \times$ midpass $_{i}+L \times$ lowpass $_{i}$ \\ highpass - set of normalized highpass values midpass - set of normalized midpass values lowpass - set of normalized lowpass values $\mathrm{H}, \mathrm{M}, \mathrm{L}$ - set of weights \\ Equation 3.2: Deviation Score formula}

\section{LocalVariability $_{i}($ size $)=\operatorname{Var}(\operatorname{scores}[i, i+\operatorname{size}])$}

Size - size of window

Var - variance of input set

Score $[x, y]$ - subset from scores starting at $x$ to $y$

Equation 3.3: Local Variability formula 


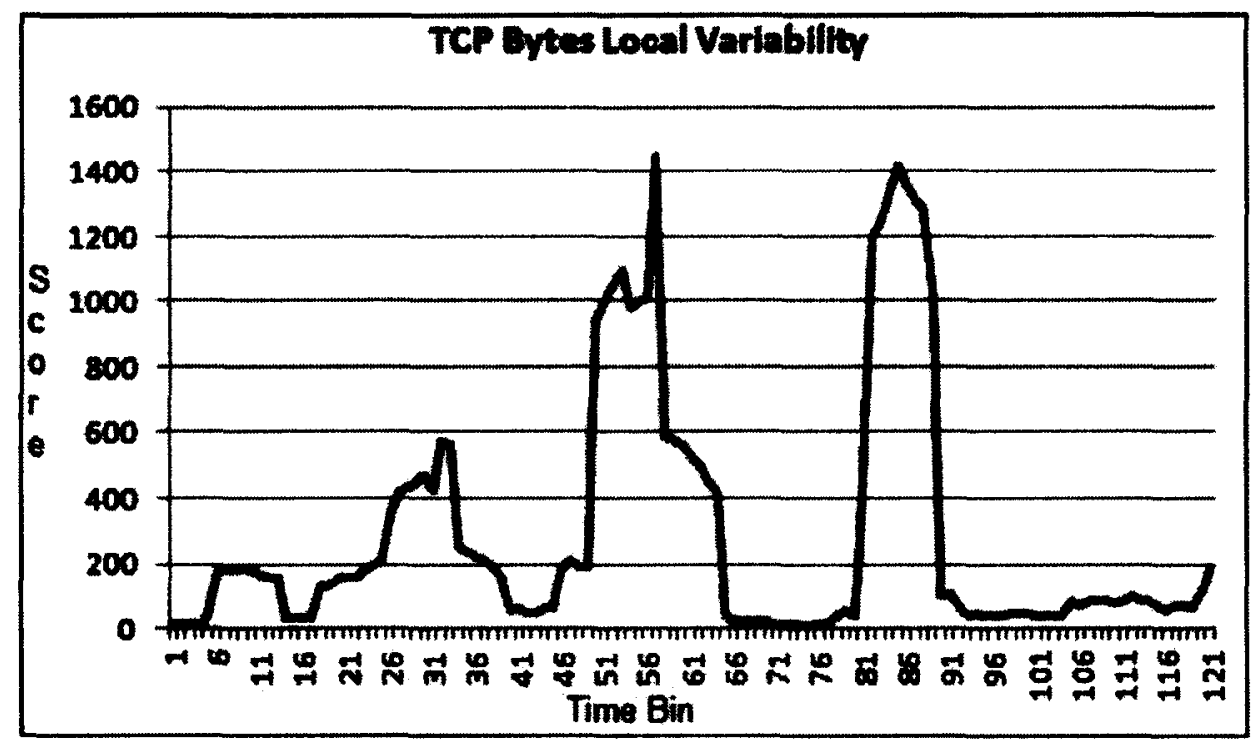

Figure 3.2: Local Variability score for March 8, 2009 with $5 \%$ infection rate

\subsection{Modified Principle Component Analysis in Anomaly Detection}

Principle Component Analysis (PCA) was designed to describe a set of observations from correlated variables into a set of new values of uncorrelated variables [13]. These variables were referred to as principle components and by definition are orthogonal. There are an equal number of principal components as original variables or features. The motivation was to create new independent components which describe the observations in more detail and uncover patterns and trends in data. For example, in a two-dimensional dataset, a line of best fit would be considered a principle component. In our application, the input into the system was the PCA time bin profiles (See Table 3.1 for an outline of the input features). As discussed in [6], applying straightforward PCA, as in [16], for anomaly detection does not account for temporal change. The type of PCA transformation applied in this work was a modified PCA approach, taken from [6]. In order to account for temporal changes, Brauckhoff et al. [6] proposed to create a spatio-temporal matrix. This matrix was a time shifted, by a variable amount of time bins, version of the original data matrix. For every feature and for every shift, a new "time shift" 
feature was created, and consisted of the subsequent observations. A number of time bins observations were dropped to compensate for the shifting (See Figure 3.3 for the time shifting illustration). Unlike wavelet analysis, PCA examined the entire input matrix as a whole, and performed an analysis on the entire dataset.

\begin{tabular}{|l|l|l|l|l|l|} 
& Time 0 & Time 1 & Time 2 & Time 3 & $\ldots$. \\
\hline Fenture 1 & A & B & C & D & $\ldots$ \\
\hline $\begin{array}{l}\text { Shifted } \\
\text { Feature 1 }\end{array}$ & B & C & D & E & $\ldots$ \\
\hline Fcature 2 & U & V & W & X & $\ldots$ \\
\hline $\begin{array}{l}\text { Shifted } \\
\text { Feature 2 }\end{array}$ & V & W & X & Y & $\ldots$ \\
\hline
\end{tabular}

Figure 3.3: Time shifting Illustration

The modified PCA analysis was applied to the entire spatio-temporal input matrix. The result was a set of ranked principal components. The first component was the most significant and the last was the least significant. Depending on the selection criteria, a number of components were selected to build the anomalous subspace. This research, examined the Top K component selection criteria, the normal distribution selection criteria and the $99 \%$ of total space selection criteria. Applying one of these criteria built a component matrix, referred to as $\mathrm{W}$. Each of the components was a column vector in the matrix W. Lakhina et al. [16] modeled the normal and residual space and projected the observations onto this space (See Equation 3.4 and Equation 3.5). For anomaly detection, the residual space was only considered. The original dataset was projected onto the residual space and a new transformed matrix was created. In order to get a measure of how anomalous each observation in time was, the Square Prediction Error (SPE) was calculated. The SPE is the Euclidean magnitude squared of the transformed 
observation in time (See Equation 3.6). Similar to the wavelet analysis, either a plot of these SPE scores could be produced for visual inspection, or threshold techniques were applied for automated classification of anomalous time bins.

$$
\text { Normal Space }=W W^{T} y
$$

$\mathrm{W}$ - column matrix of selected components $\mathrm{y}$ - original data observations

Equation 3.4: Normal Space formula [16]

\section{Abnormal Space $=I-W W^{T} y$}

$\mathrm{W}$ - Column matrix of selected components I - Identity matrix of size I $y$ - Original data observations

Equation 3.5: Abnormal Space formula [16]

$$
\begin{gathered}
\mathbf{S P E}_{\mathbf{I}}=\|\tilde{\mathbf{C X}} \mathbf{i}\| \\
\text { SPEi - Square Prediction error at observation } \mathrm{i} \\
\mathrm{C}-\text { Matrix of anomalous subspace components } \\
\mathrm{Xi}-\text { An observation, column vector from original dataset }
\end{gathered}
$$

Equation 3.6: Square Prediction Error (SPE) formula [16] 


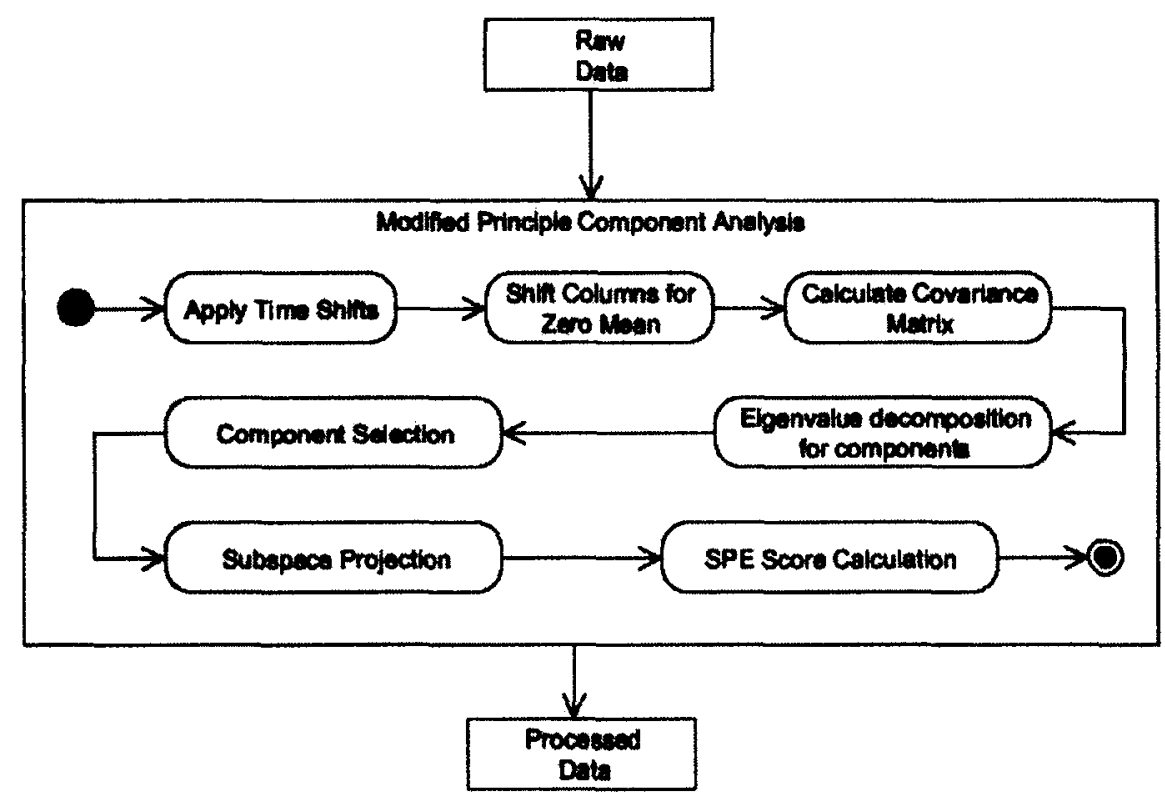

Figure 3.4: Activity Diagram of Modified PCA approach

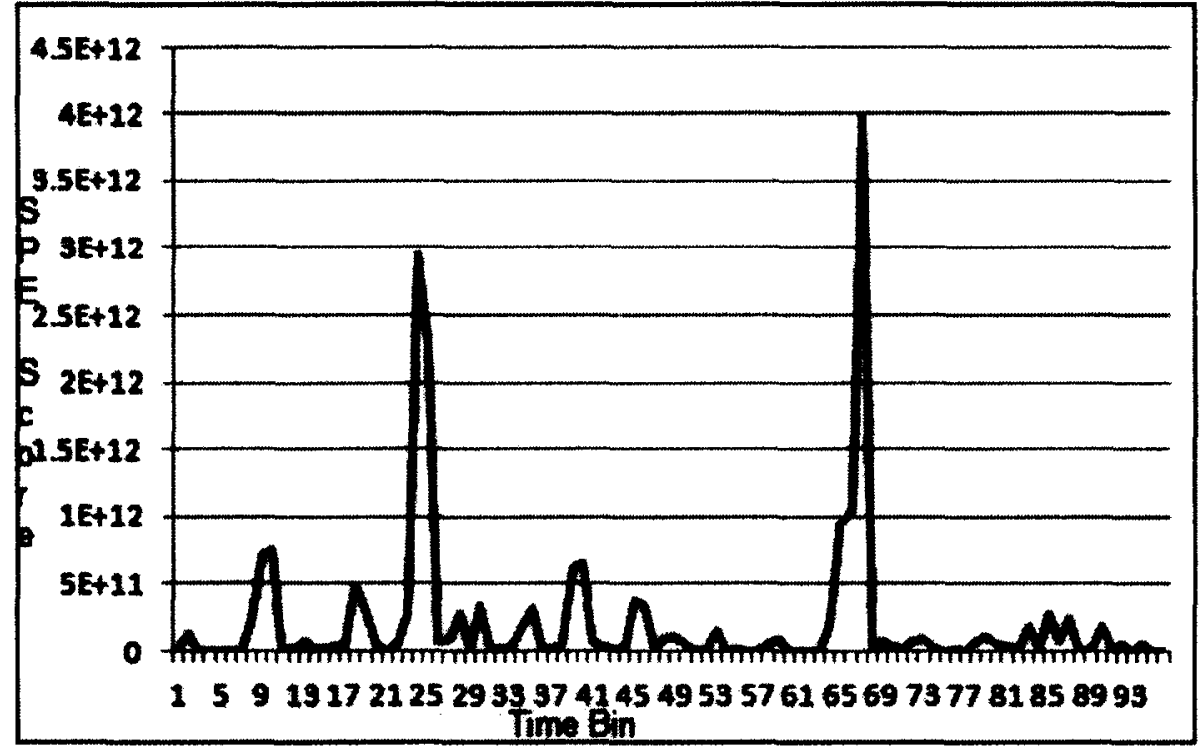

Figure 3.5: SPE score of March 8, 2009 for 5\% infection rate

\subsection{A Hybrid Analysis in Anomaly Detection}

The hybrid analysis is a combination of the traditional Principal Component Analysis approach, as presented in [16], and Haar wavelet analysis, as used in [7]. Each of the approaches 
was described in sections 3.2 and 3.3 respectively. The hybrid approach uses the statistical and spectral analysis in a complementary fashion, and attempts to be less parameter dependant. The hybrid approach first aims to examine the entire dataset as a whole, as in PCA. Subsequently, a detailed localized frequency analysis to identify potential anomalous time bins is applied. In our application, the input into signal was the PCA time bin profile matrix (See Table 3.1 for an outline of the input features). Creating a time shifted spatio-temporal input matrix, as adopted in [6], was not considered. Next, the Haar wavelet analysis, examines the behaviour over time, and the concerns which shifting addressed are considered then.

The hybrid algorithm first applied PCA analysis to obtain a set of components. As in [16], the normal and residual spaces were modeled, and as before, only the residual space was considered. Unlike previous analysis in this research, for simplicity, only the single most significant component was considered. It was determined that the most significant component occupied a majority of the space. Limiting the selection criterion to "Top $K=1$ components" reduced the dependency on user specified parameters. As before, the original data input was projected onto the residual space in order to produce a transformed dataset for further analysis. The transformed dataset was comprised of the same amount of time bin observations as the input data. Applying the projection did not drop or create any new features. It did transform the existing ones, and strip them of their semantic meaning. The semantic meaning may have been lost; however, the new features were more representative of the behaviour and were able to potentially reveal patterns or trends.

The Haar wavelet analysis examines the degree of change of a single feature set at a time. This approach strives to be independent from user input parameters. The hybrid algorithm applies a selection criterion between features, based on variance. Variance is a measure of 
spread within a dataset. A dataset may have the same average, but the dataset with the greater variance is more spread out (See equation 3.7). In the hybrid approach, every feature dataset is examined and the feature with the greatest variance is selected for wavelet analysis. The motivation was that the most spread out data had the greatest potential for large changes over time. Next, as outlined in [7], the Haar wavelet high pass filter, low pass filter and mid pass filter are each independently applied to the input data. Each of the filtered dataset is normalized (see Equation 3.1) and a score, similar to deviation score, is calculated. This score is a summation of weighted normalized frequency values for each point in time. Local variability was not considered in order to be independent from user defined parameters. The score highlights the descriptive properties of the filtering and was sufficient enough to characterize the behaviour of the data. Examining data over a subset is offloaded to the threshold phase. Yet, the Haar wavelet filtering produced an output of negative values which is not semantically meaningful in the network traffic domain. In this case, the absolute difference, or delta, between two time bin scores (See equation 3.8) is examined. Either a plot of these delta scores was produced for visual inspection, or threshold techniques were applied for automated classification of anomalous time bins.

$$
\begin{aligned}
& \operatorname{Var}(x)=\frac{1}{N} \sum_{i=1}^{N}\left(x_{i}-\mu\right)^{2} \\
& \text { Equation 3.7: Variance formula } \\
& \text { DeltaScore }_{i}=\mid \text { score }_{i}-\text { score }_{i+1} \mid \\
& \text { Score }_{i} \text { - the anomalous score for a specific time bin } \\
& \text { Equation 3.8: Delta score formula }
\end{aligned}
$$




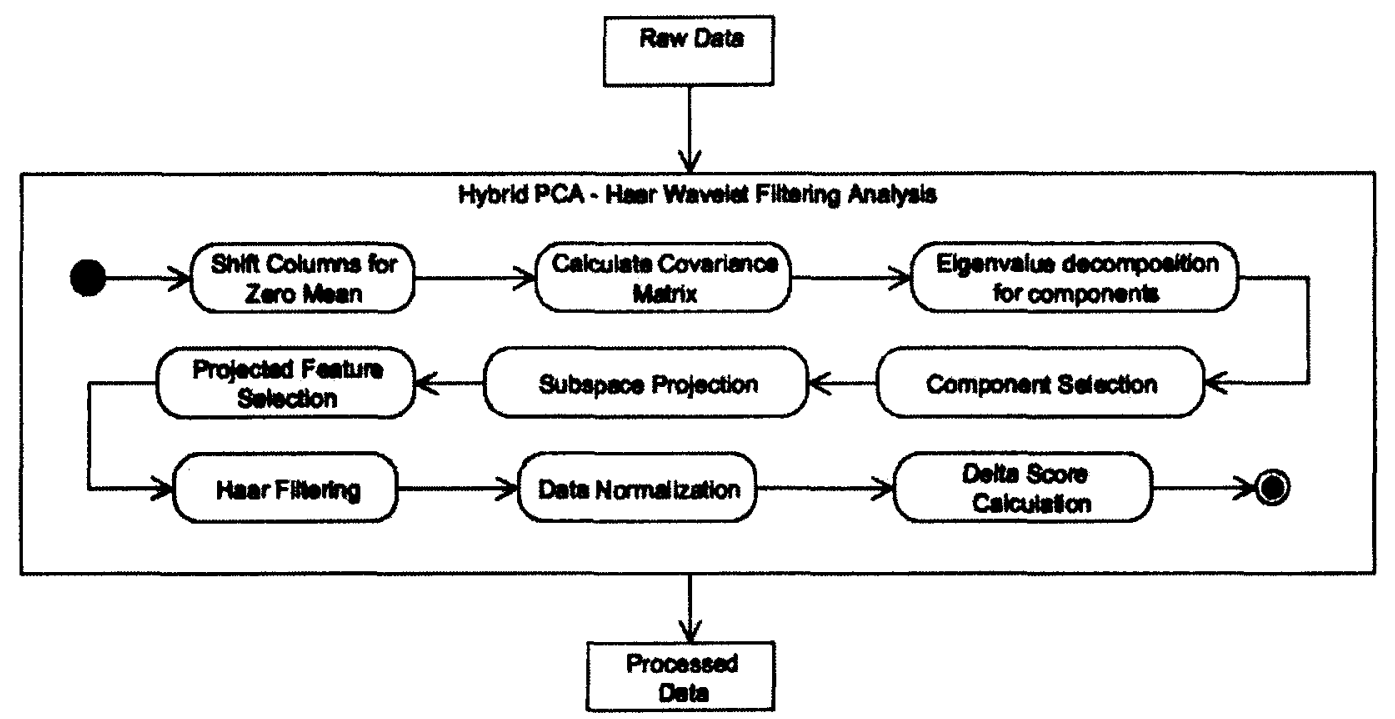

Figure 3.6: Hybrid PCA-Haar Wavelet Filtering activity diagram

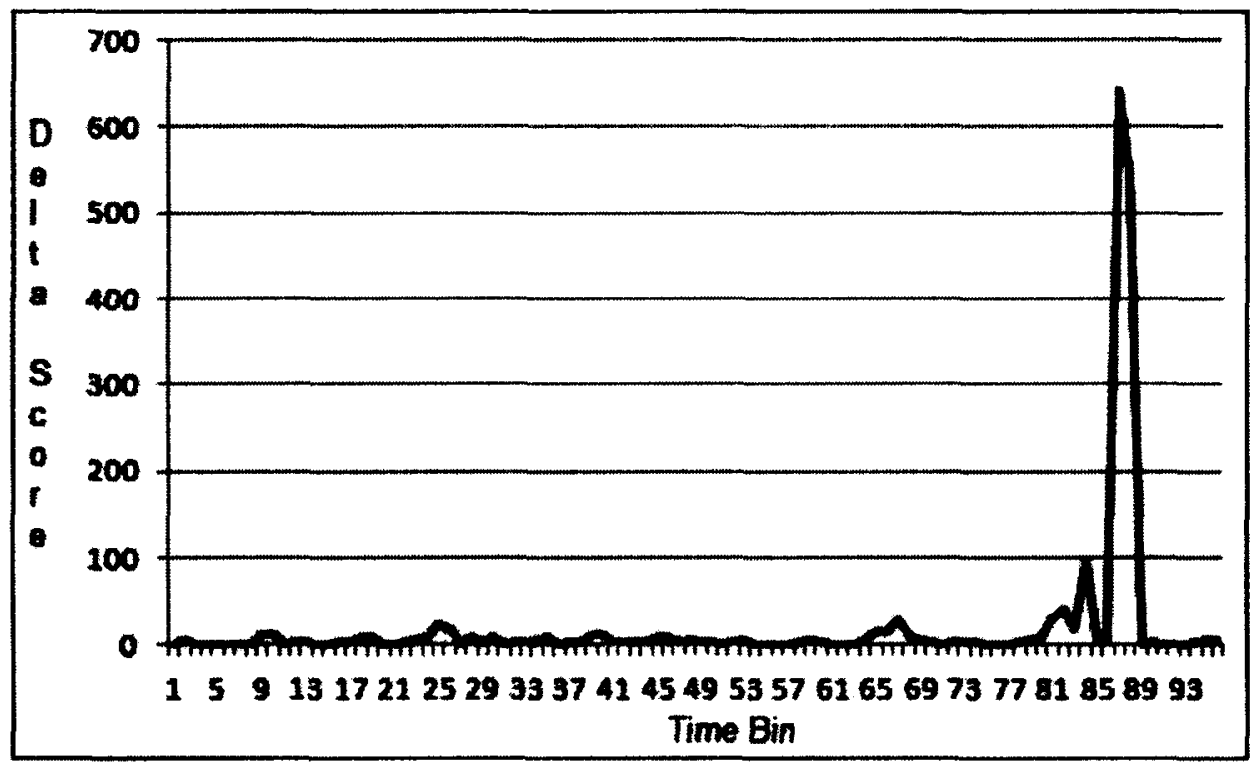

Figure 3.7: Delta score for March 8, 2009 for $5 \%$ infection rate

\subsection{Threshold Techniques}

A visual plot was a quick and easy way to present data. Yet, automating a specific task has many advantages, such as decreased detection time, reduction of human error and decreased 
operational complexity. The following subsections discuss various threshold techniques applied to the output plots of each of the anomaly detection methods.

\subsubsection{Average}

The quickest and simplest threshold calculation is the average. This calculation considers the entire set of scores and calculates the statistical mean (See Equation 3.9). However, an overwhelming majority of flows are less than one minute in length. It is problematic calculating an average from an analysis over a day or two. The behaviour the next day may be very much different and giving equal consideration to each time bin score may not be appropriate. A moving average, of size $\mathrm{N}$ observations, is a way of handling this range in time. The statistical mean for the first $\mathrm{N}$ elements is calculated and is the threshold value for the first $\mathrm{N}$ time bins. Then, the window slides over by one and the threshold value for time bin $\mathrm{N}+1$ is the average. This repeats until the window reaches the end of observations (See Equation 3.10). Any score greater than either than the total average or moving average at this point in time is flagged as potentially anomalous for the network administrator.

$$
\begin{gathered}
\text { Static Average }=\frac{\sum \text { scores }}{n} \\
\text { score }- \text { set of scores } \\
\mathrm{n} \text { - number of elements in set } \\
\text { Equation 3.9: Static Average formula } \\
\text { Moving Average }=\frac{\sum \text { scores }[i, i+n]}{n} \\
\text { score[x,y]- set of scores starting at } \mathrm{x} \text { to y } \\
\mathrm{n}-\text { number of elements in set } \\
\text { Equation 3.10: Moving Average formula }
\end{gathered}
$$




\subsubsection{Sliding Weighted Average}

As discussed in the previous section, it may not always be appropriate to give equal weighting to each time bin score value. The sliding weighted average is alternative to the sliding moving average. This approach applies a weighting to each time bin score value and considers calculates the statistical mean. Each observation has a weight that is multiplied to the observation value. The weighting has an initial weighting of 1.0 and decrements evenly to 0 . Similar to the moving average described in section 3.10 , a window size, of $\mathrm{N}$ observations, is provided and those elements in the window are considered. This repeats until the window reaches the end of observations (See Equation 3.11). Any score which is greater than this values determined at this point in time is flagged as potentially anomalous for the network administrator.

$$
\begin{gathered}
\text { WeightedMovingAverage }=\frac{\sum_{j=0}^{n} W_{j} \times \operatorname{scores}[i, i+n]}{n} \\
\begin{array}{c}
\text { score[x,y]- set of scores starting at } \mathrm{x} \text { to } \mathrm{y} \\
\mathrm{n}-\mathrm{number} \text { of elements in set } \\
\mathrm{W}-\text { set of weights }
\end{array} \\
\text { Equation 3.11: Weighted Moving Average formula }
\end{gathered}
$$

\subsubsection{Average without outliers}

Similar to the previously discussed anomaly detection algorithms, this threshold technique attempts to separate normal behaviour and residual values. The motivation is to build a curve which represents the normal behaviour and act as a threshold. There are two user defined parameters: the number of observations to be considered at a time, $N$ and the growth tolerance, $G T$. The growth tolerance, $G T$, defines how much larger the time bin score can be from the threshold value. The first time bin score is the first threshold value. The average of the 
first two time bin scores is the second threshold value. The average of the first three time bin scores is the third threshold. This continues up until the first $N$ threshold values are determined. The average of the first $N$ many scores is considered the running threshold. If the next element is greater than the running threshold plus the tolerance, the corresponding time bin is flagged as potentially anomalous. Also, in this case, running threshold is not updated, and the threshold for that time bin is the current running threshold. However, if the element is less than or equal to the running threshold plus the tolerance, the running threshold is updated. The update calculates the average over the $N$ elements, considering the new score and dropping the oldest score. The threshold value for that time bin is the new updated threshold value. Each element is considered one by one until all data has been examined. Any time bin scores greater than the corresponding threshold value are flagged as potentially anomalous for the network administrator. 


\subsection{Prototype Design and Implementation}

A prototype test bed was developed to perform experiments. The algorithms and various functions outlined in previous chapters were implemented in order to obtain experimental results perform analysis. The prototype anomaly detection test bed is comprised of two components; the front end user interface and the backend server. It is implemented with client-server architecture. The front end is a browser based client interface and was implemented using an interactive open-source Web development framework, called Adobe Flex [34]. The browser is the responsible for handling user commands, making requests to the server and presenting data. The back end server is an open-source, Java based, web server called Apache Tomcat [35]. This server handles data lookup requests and applies various algorithms to specified datasets. The server performs various operations requested, and responds to the front end. The user is able to view the results analyze them and execute more operations (See Figure 4.1 for a system diagram). This chapter provides a high level overview of the test bed and describes the design and implementation of each of the two components.

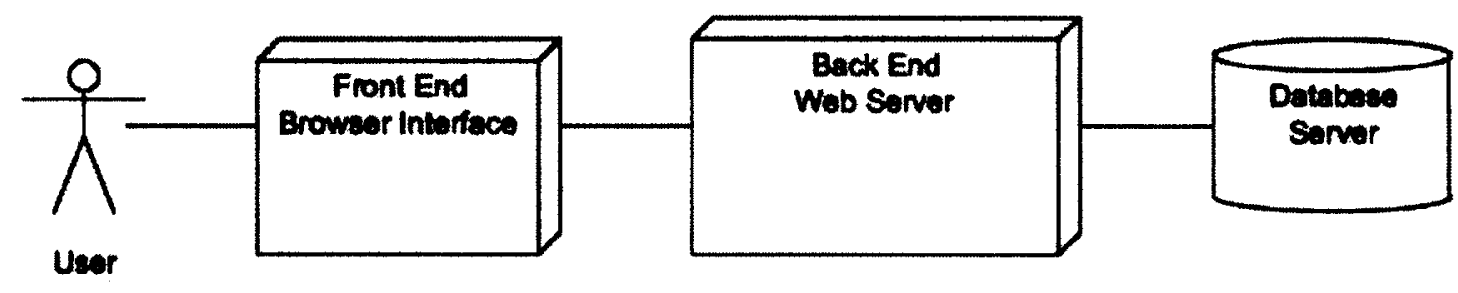

Figure 4.1: Test bed system diagram

\subsection{Front-end Interface}

Typically, desktop applications are stand alone programs which are platform dependant and sensitive to a number of environment parameters, such as hardware specifications, operating 
systems, etc. Applications can become complex to develop and maintain. Conversely, web pages are tremendously easy to implement. The framework front end is browser based, built using the Adobe Flex Web framework, and is very quick and easy to implement. The Adobe Flex framework is an open source technology used to create rich Internet applications. These applications are web pages that are dynamic, their content is reloadable and they act like desktop applications. Adobe Flex supports traditional Web framework functions such as POST and GET requests to servers. Furthermore, it provides a number of graphs and widgets which bind with XML data to render detailed illustrations. Adobe Flex is a powerful tool, does not require extensive programming experience to use and satisfies all of the client interface requirements. [34]

The network administrator operates the browser based front end. The interface enables the user to perform and examine all phases of the proposed anomaly detection system (See Figure 4.2 for a use case diagram of anomaly detection test bed). The operator can load raw network traffic data into the system, and drop any existing datasets. The operator can create subsets of data for analysis from loaded traffic data. These subsets can contain a desired infection ratio during a specified time segment. Data profiles can be created from the subsets and preserved for future analysis. The anomaly detection algorithms, described in chapter 3 , can be applied to data profiles, and the results are visualized in a plot for manual inspection (See Figure 4.3 for a screen capture of a plot). For the results, in some cases, higher scores can overwhelm the behaviour of smaller scores. The user has the ability to remove specific scores such that the plot can be amplified on the remaining data. For manual investigation, the threshold function is plotted on top of the results. The program supports manual shifting of the threshold. The various threshold techniques, as outlined in section 3.5 , can also be applied to 
provide an automatic classification of time bins (See Figure 4.4 for a screen capture). The Kyoto2006+ is a labeled dataset and the evaluation scores can be calculated to provide the user a scalar measure of how well the approach performs under the specified conditions (See Section 5.2 for an outline of the evaluation measures). The front end is comprehensive enough such that the user can perform their own inspection, or rely on the system automated results.

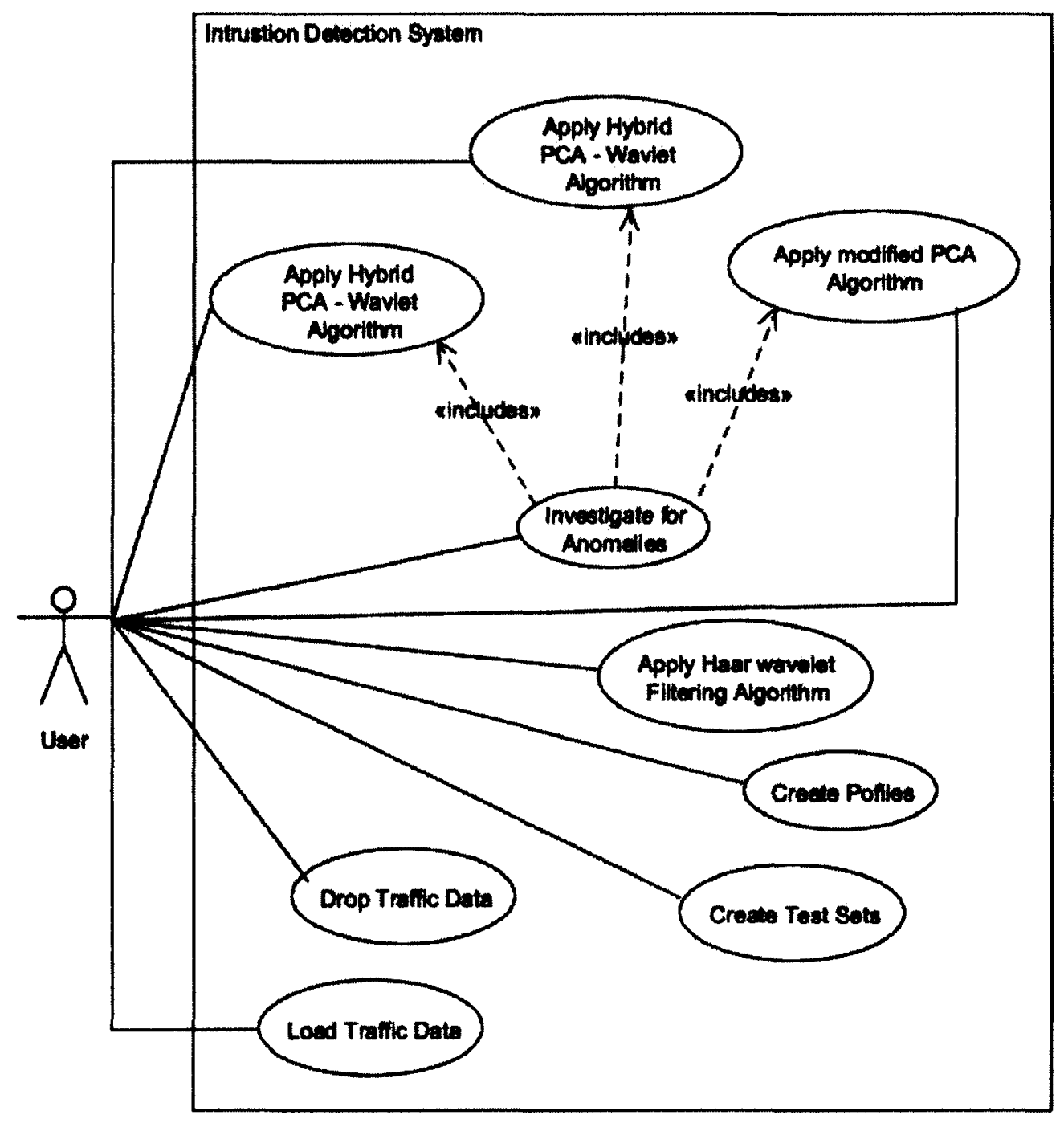

Figure 4.2: Use case diagram of test bed system 


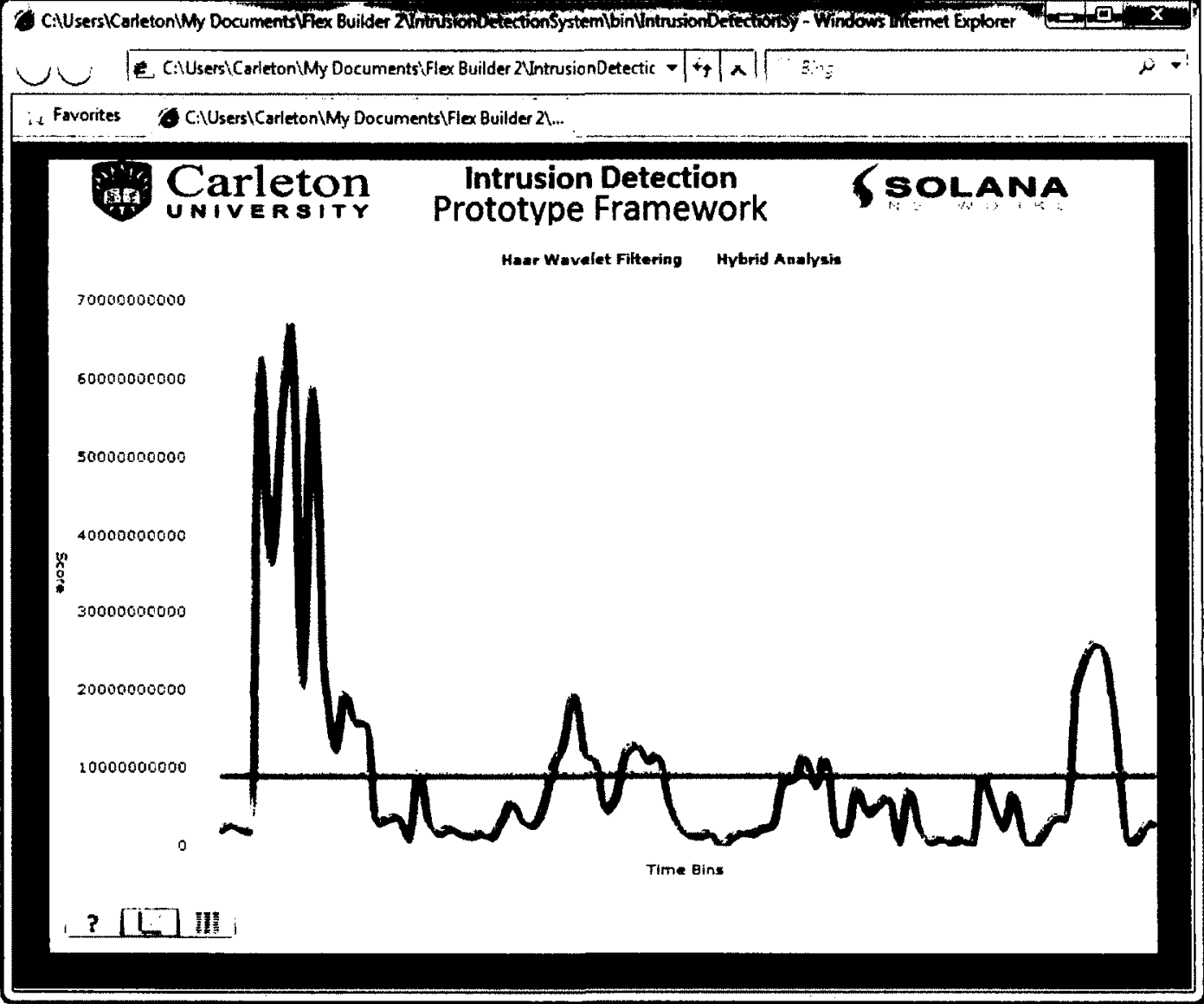

Figure 4.3: Screen capture of SPE scores plotted from test bed 


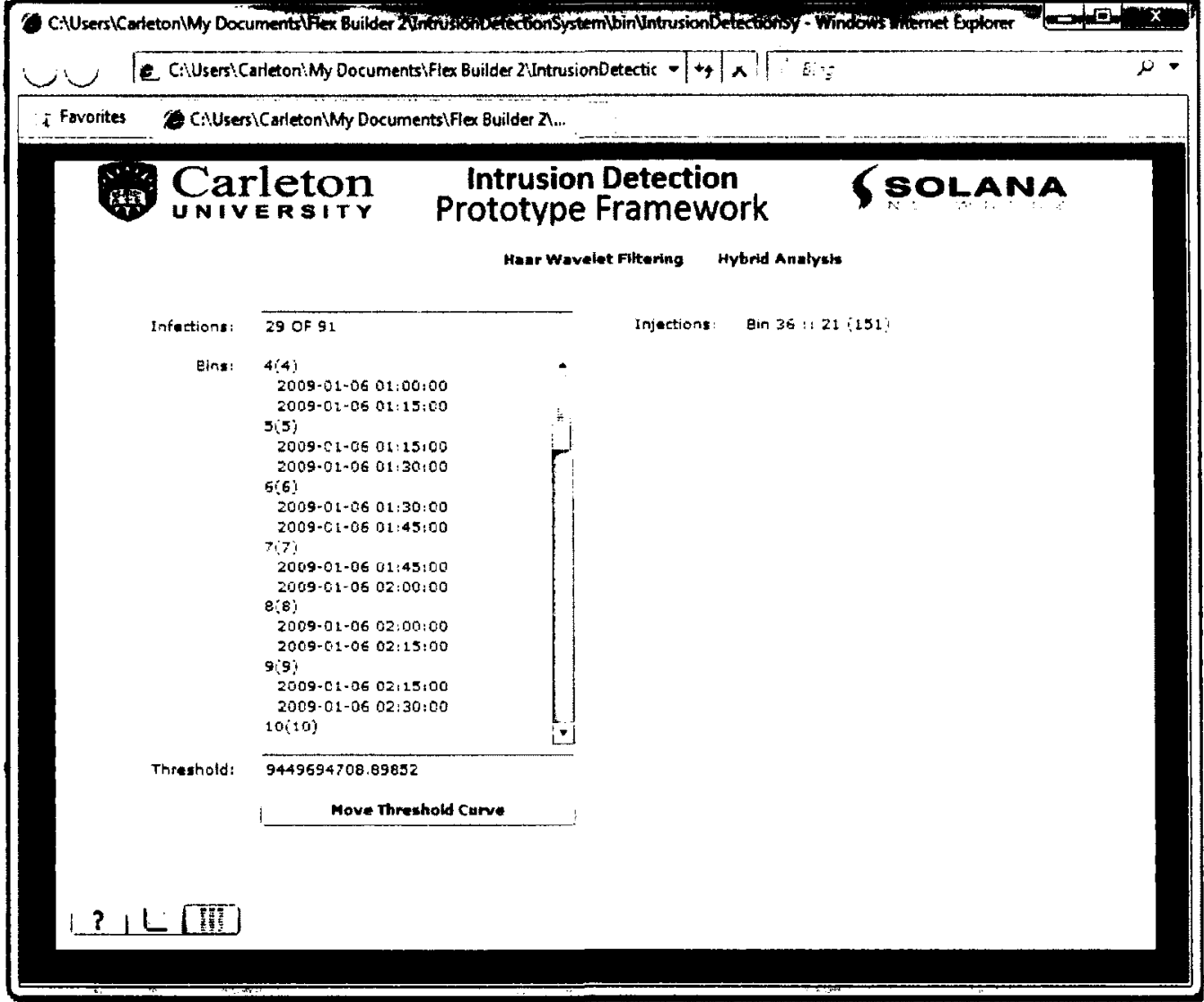

Figure 4.4: Screen capture of information panel from test bed

\subsection{Back-end Server}

The back end serves the client user interface. The server sits idle until requests are made and responds to them in an XML format. The back end is an Apache Tomcat Web server, which implements Java servlets. To handle incoming http requests, for a pure Java Web serving solution [35]. All Java classes, interfaces and libraries are created, compiled, packaged together and then deployed on the Apache Tomcat server. Java satisfies the requirements for this application, as it is a powerful platform with the ability to connect with a local file system, a database and perform CPU intensive computations.

The server interfaces with various system components. As the network traffic data comes in text files, the system interfaces with the file system and a database. A database is used 
because it provides support for complex queries and enables specific data to be accessed easily. The server loads network traffic data into a database. Additionally, it poses queries to the database in order to create the profiles and save them into text files for future analysis. All of the anomaly detection algorithms described in Chapter 3 are implemented, and can be applied to a user specific dataset. In order for increased system stability, open source libraries, such as a Matrix library and a Haar wavelet filtering library, are leveraged (See Figure 4.5 for a class diagram of the system). Open source libraries from credible sources, such as Google projects, are preferred because they are maintained by a community and have been used extensively. The intense computations are preformed on the server side to remove workload from the user interface. Java is a powerful platform which is more appropriate to perform CPU intensive computations over a Web scripting language. The threshold techniques, evaluation operations and other utility functions were implemented in order to support all the features of the client interface. As required by Adobe Flex, all server responses are formatted in the XML format.

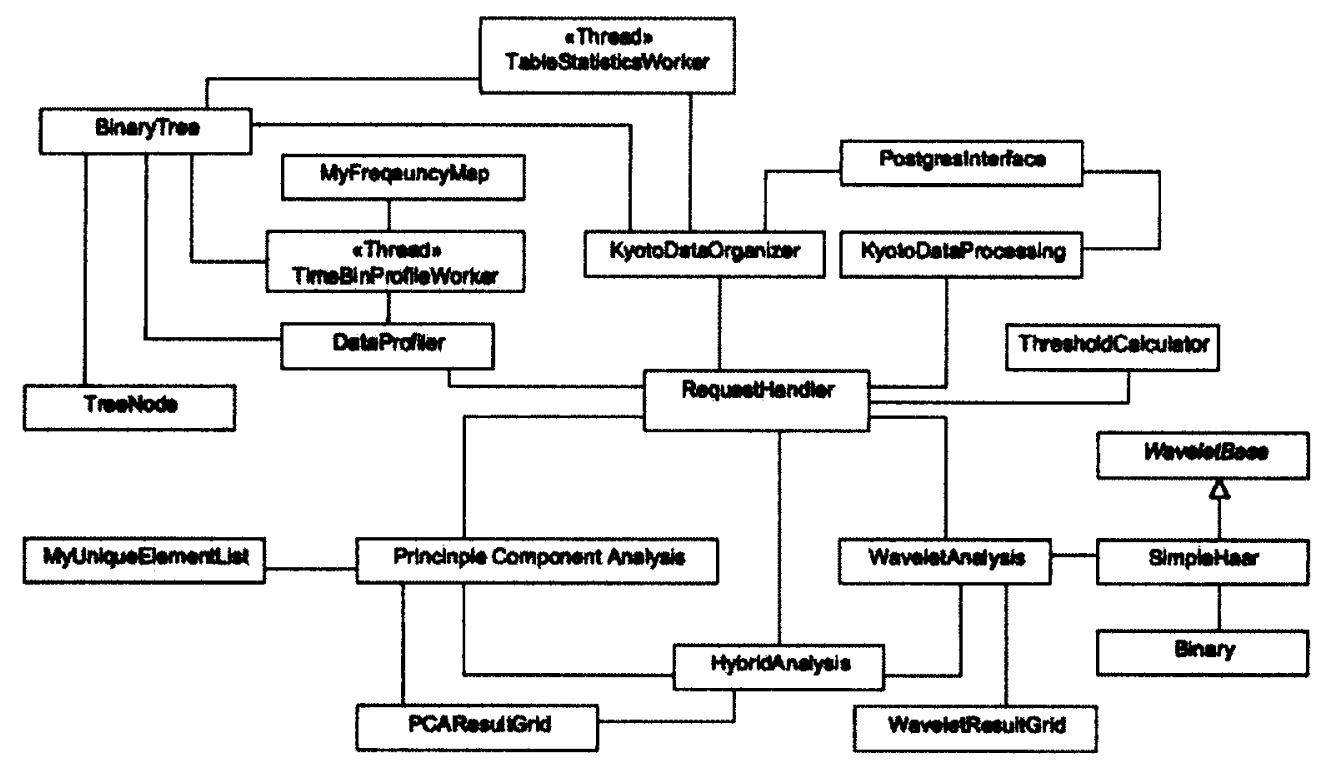

Figure 4.5: Class diagram of backend server from test bed 


\subsection{Experiments and Evaluation}

This chapter presents the experiments conducted in this research, including the data sources used and data profiling. Furthermore, it outlines the intrusion detection algorithm step, threshold / anomaly detection step and lastly, the evaluation.

\subsection{Experiment Overview}

There were a number of experiments conducted with the anomaly detection test bed to determine the performance of the three anomaly detection algorithms presented in Chapter 3 . Prior to applying the algorithms, datasets for testing needed to be created and profiled. A number of specific dates and infection times were selected for testing. These test sets were created and then both the PCA and the Wavelet profiles were extracted. The three algorithms were applied independently and using various parameters. Threshold techniques were applied to determine the potentially anomalous time bins. As the test sets were labeled, evaluation techniques were used to evaluate the performance of the intrusion detection algorithms.

\subsection{Experimental Setup}

This section outlines how the experiments were constructed and provides the rationale for the experiment decisions made. First, data setup is discussed and then evaluation techniques are described.

\subsubsection{Data Source Setup}

As discussed in section 3.1, the Kyoto2006+ data source was used in experimentation [8]. The Kyoto2006+ dataset provides session data for each day, starting in November 2006 until the end of August 2009. The volume of network traffic sessions increased with time. For example, 
November 2006 had 1,223,899 sessions, November 2008 had 2,374,504 sessions and July 2009 had $3,870,725$ sessions. The data available in 2009 was the largest, which is why analysis began in January 2009.

As per the Kyoto2006+ specification, there are three features which outline the output of existing open-source detectors [8]. Also, the label feature indicates the authors' belief about the particular session is anomalous or not. For our research, normal flows were defined as flows the authors labeled as normal (1) and raised no detector alerts. Furthermore, infected flows were flows labeled anomalous $(-1)$ by the authors and raised an alert with at least one of the three detectors. This approach used the detectors as validation to remove any uncertainty from the labeling. The session would more likely be normal or anomalous if all methods agreed.

One major limitation from this dataset is the amount of flows labeled as -1. Examining the flow counts (See Table 5.1), it can be observed that there is an overwhelming majority of these flows and this issue has been raised by Song et al. [26]. The aforementioned definition of anomalous flows was applied, and the number reduced so much that it became the minority. Next, the definition of normal flows was applied and observed that the number of normal flows also reduced. The result was that there were many more normal flows than anomalous, and the total amount of data was less than before. The approach used in this research, similar to [26], was to build a test set using some normal and some anomalous flows.

Our analysis was applied on 24 hours at a time. Throughout the 2009 dataset, the duration of sessions less than one minute was $98 \%$. Performing analysis on 24 hours at a time would maintain small enough time bin duration such that anomalies would not be hidden. Although other works, such as [6] and [7], used a different data source with different features, they still used analysis over one day. A total of 16 24-hour time ranges, which had different 
behaviours, were selected. This research considered two attack characteristics: length of attack time and intensity of attack. Hence, experimentation was performed over four different infection scenarios:

- $\quad$ short length and high intensity.

- long length and high intensity.

- short length and low intensity.

- short length and high intensity.

The base case with no infection, $0 \%$ infection rate, was considered. For low infection rates, $5 \%, 10 \%$, and $20 \%$ infection were created. For high infection rates, $75 \%, 100 \%$ and $150 \%$ infection were created (See Equation 5.1 for infection rate calculation). A total of 16 different time ranges, four for each scenario, were picked out. These ranges varied in days of the week, and actual time of day in which the attack occurred (See Table 5.2 for a breakdown).

\begin{tabular}{|c|c|c|c|c|c|}
\hline Month & $\begin{array}{c}\text { Total } \\
\text { Flows }\end{array}$ & $\begin{array}{c}\text { Total } \\
\text { Normal } \\
\text { (label = 1) }\end{array}$ & $\begin{array}{c}\text { Total } \\
\text { Abnormal } \\
\text { (label =-1) }\end{array}$ & $\begin{array}{c}\text { Total our normal } \\
\text { (label=1 and no } \\
\text { alerts) }\end{array}$ & $\begin{array}{c}\text { Total our abnormal } \\
\text { (label = -1 and at least } \\
\text { one alert) }\end{array}$ \\
\hline January & $3,670,176$ & $2,216,820$ & $1,453,356$ & $2,177,273$ & 129,571 \\
\hline February & $3,321,532$ & $1,947,619$ & $1,373,913$ & $1,911,817$ & 113,398 \\
\hline March & $3,398,114$ & $1,861,103$ & $1,537,011$ & $1,828,827$ & 139,291 \\
\hline April & $3,767,136$ & $2,637,097$ & $1,130,039$ & $2,534,233$ & 106,136 \\
\hline May & $3,747,527$ & $2,153,355$ & $1,594,172$ & $2,108,499$ & 107,484 \\
\hline June & $3,743,655$ & $1,852,554$ & $1,891,101$ & $1,807,121$ & 101,633 \\
\hline July & $3,870,725$ & $2,086,698$ & $1,784,027$ & $2,026,854$ & 81,632 \\
\hline August & $3,710,941$ & $1,640,651$ & $2,070,290$ & $1,568,229$ & 223,476 \\
\hline
\end{tabular}

Table 5.1: Kyoto2006+ flow count breakdown per month 


\begin{tabular}{|c|c|c|}
\hline Dates & Start Time & Analysis Duration \\
\hline Short Duration and Low Infection Rates & & \\
\hline Tuesday, January 6, 2009 & $0: 00$ & 24 hours \\
\hline Sunday, March 8, 2009 & $22: 00$ & 24 hours \\
\hline Sunday, April 26, 2009 & $20: 00$ & 24 hours \\
\hline Tuesday, May 5, 2009 & $21: 30$ & 24 hours \\
\hline Short Duration and Low Infection Rates & & \\
\hline Friday, February 20, 2009 & $0: 00$ & 24 hours \\
\hline Thursday, March 19,2009 & $0: 00$ & 24 hours \\
\hline Thursday, May 28, 2009 & $18: 00$ & 24 hours \\
\hline Tuesday, July 14, 2009 & $19: 00$ & 24 hours \\
\hline Short Duration and High Infection Rates & & \\
\hline Thursday, January 15, 2009 & $0: 00$ & 24 hours \\
\hline Wednesday, April 1, 2009 & $20: 30$ & 24 hours \\
\hline Monday, June 22, 2009 & $0: 00$ & 24 hours \\
\hline Sunday, July 5, 2009 & $0: 00$ & 24 hours \\
\hline Long Duration and High Infection Rates & & \\
\hline Monday, February 9, 2009 & $21: 00$ & 24 hours \\
\hline Wednesday, June 10, 2009 & $22: 00$ & 24 hours \\
\hline Sunday, August 9, 2009 & $20: 00$ & 24 hours \\
\hline Friday, August 28, 2009 & $19: 00$ & 24 hours \\
\hline
\end{tabular}

Table 5.2: Dates in experimentation by scenario and analysis duration

$$
\text { InfectionRate }=\frac{\# \text { of injected Abnormal Flows }}{\text { Equation 5.1: Infection rate formula }}
$$

In order to maintain statistical independence, ten test sets were created. Similar to Song et al. [26], all normal flows and a subset of anomalous flows to create the desired infection rate in a test set were used. The test sets were derived from the original primary dataset. At each of the 16 time ranges, all the normal flows from the main collection were randomly and evenly distributed among the test sets. Each flow would be in only one test set, and all test set sizes had the same amount of flows. Next, depending on testing scenario, or desired infection ratio, a number of anomalous flows were injected. The flow characteristics were not changed. Due to 
the limited number of available anomalous flows, membership was not restricted to one test set. From these test sets, profiles were extracted and the algorithms were applied.

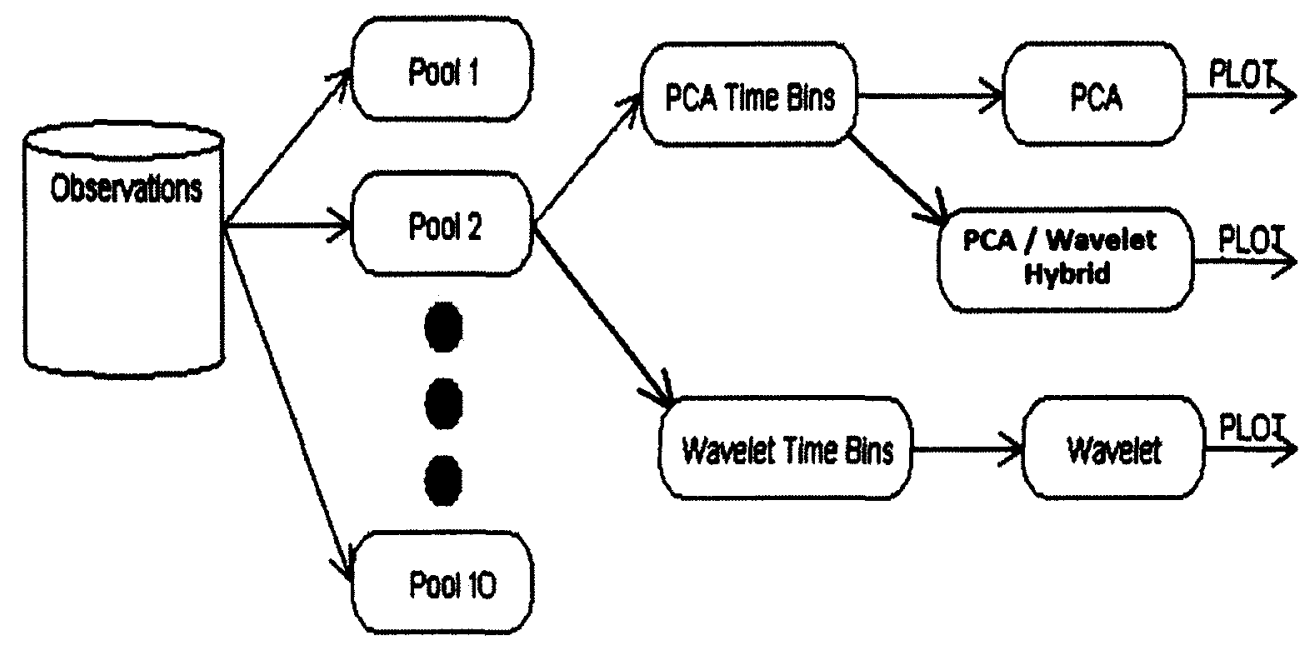

Figure 5.1: Experiment high level diagram

\subsubsection{Performance Measurement Setup}

The Kyoto2006+ is a labeled dataset, so it is possible to measure how well the algorithms perform. Two measurements were used to evaluate the performance of the algorithms: accuracy and precision. Accuracy is defined as how correctly the time bins are classified. It was measured using a hit miss count which counted the number of correct and incorrect classifications. The definition of precision is how exactly the algorithm can classify time bins. It was measured using a single scalar value to show how well the detection method performs.

\subsubsection{Hit and Miss Count}

The hit miss count compares the presence of anomalous flows within time bins. As part of the profiling phase, the number of anomalous flows and the number of normal flows per time bin preserved in the form of a list. If the anomalous flow count in a time slice was greater than 
zero, the bin was classified anomalous. Otherwise, it would be classified normal. After applying the anomaly detection algorithms and using the threshold techniques, a list of presumed abnormal and normal time bins was produced. The actual list and the experimental list were compared and the hit and miss counts were calculated. The hit count was the number of time slices in which the labels matched up in both lists. Conversely, the miss count was the number of time slices in which the labels did not match. These counts provide an indication of how accurately the algorithm performed.

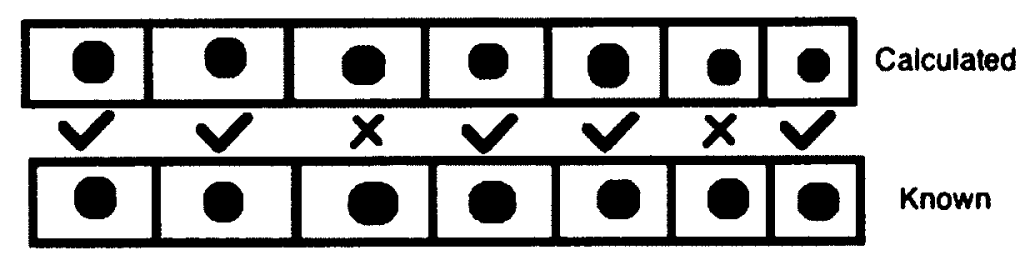

Figure 5.2: Hit and miss count illustration

\subsubsection{Hit and Miss Score}

The hit and miss score is a credit scheme where different amounts of credits are awarded and taken away based on the situation. There is some uncertainty with the information provided by the Kyoto2006+ detectors. The programs are primarily signature based detection systems and may miss some anomalies [4]. The credit score aims to accommodate for this uncertainty (See Table 5.3 for an outline of the credit scoring key). Similar to the hit and miss count, the number of anomalous and normal flows for each time slices was preserved. After applying the algorithms, a list of presumed abnormal and normal time bins was produced. Each time bin flagged as abnormal was compared with the known counts of normal and abnormal flows. In the most obvious case, where there are all infections and no normal flows, two credits are awarded. If there are no flows at all, two credits are taken away. No reward or penalty was awarded if only normal flows were present. This may be a scenario in which the authors of the Kyoto2006+ 
could have incorrectly classified the bin. If a bin with normal and anomalous flows is classified, then one credit is awarded. These scores provided an indication of how precisely the algorithm performed. A higher score indicates a more precise analysis.

\begin{tabular}{|c|c|c|}
\hline Anomaly Count & Normal Count & Credits \\
\hline 0 & $>0$ & 0 \\
\hline 0 & 0 & -2 \\
\hline$>0$ & $>0$ & +1 \\
\hline$>0$ & 0 & +2 \\
\hline
\end{tabular}

Table 5.3: Credit Score Breakdown

\subsection{Experimental Procedure}

For each method, a number of experiments were conducted. The parameters considered for each method include the combination of the infection rate, the four scenarios described in section 5.2.1, and the time ranges. Table 5.4 summarizes the tunable parameters and default values adopted for the experiments. The following summarizes some experimental conditions:

- Infection rate: $0 \%, 5 \%, 10 \%, 20 \%$ and $75 \%, 100 \%, 120 \%$

- Length of attack time: short (45 minutes) and long (150 minutes)

- Intensity of attach: low $(0 \%, 5 \%, 10 \%, 20 \%)$ and high $(75 \%, 100 \%, 120 \%)$

- Number of time ranges: 16 


\begin{tabular}{|c|c|c|c|}
\hline Algorithm & Parameter & Values & Default Value \\
\hline \multirow[t]{2}{*}{$\begin{array}{l}\text { Modified } \\
\text { PCA }\end{array}$} & $\begin{array}{c}\text { Component Selection } \\
\text { Criteria }\end{array}$ & $\begin{array}{c}\text { Subspace method, } \\
\text { Top } k=1 \text {, } \\
\text { Variance }>1 \text { method }\end{array}$ & $\begin{array}{l}\text { Subspace } \\
\text { method }\end{array}$ \\
\hline & Number of Time Shifts & $1,2,3$ & 1 \\
\hline \multirow[t]{2}{*}{$\begin{array}{c}\text { Haar } \\
\text { Wavelet } \\
\text { Filtering }\end{array}$} & $\begin{array}{c}\text { Input data size / } \\
\text { Boundaries }\end{array}$ & $\begin{array}{c}\text { Input size / filtering levels } \\
128 /(2,3,2),(3,2,2) \\
256 /(3,2,3),(3,3,2) \\
512 /(3,3,3),(4,3,2)\end{array}$ & $128 /(2,3,2)$ \\
\hline & Local Variability Weights & $\begin{array}{l}(5,1,0), \\
(3,2,0)\end{array}$ & $(5,1,0)$ \\
\hline BOTH & Threshold method & $\begin{array}{l}\text { Moving Average without } \\
\text { outliers, } \\
\text { Moving Average, } \\
\text { Static Average, } \\
\text { Weighted Moving Average }\end{array}$ & $\begin{array}{l}\text { Moving Average } \\
\text { without outliers }\end{array}$ \\
\hline
\end{tabular}

Table 5.4: Outline of parameters used in experimentation

\subsubsection{Test Set Creation}

Analytics were performed on each the available data for each month. The number of anomalous and normal flows every 15 minutes was counted. For each of the scenarios, listed in section 5.2.1, four time ranges were selected. A total of two days from each of the 8 months in 2009 were examined. When examining for appropriate dates, short was defined as three 15 minute time bins, and long was considered as ten 15 minutes time bins. A low amount of flows were considered to be able to create at most a $20 \%$ infection rate. A high amount of flows was considered to be able to create at most a $20 \%$ infection rate. The Modified Principle Component Analysis and Haar Wavelet Filtering algorithms each have their own set of tunable parameters (See Table 5.4 for an outline of parameters used in experimentation).

\subsubsection{Modified Principle Component Analysis Experiments}

A number of experiments with the Modified PCA were conducted. In addition, the selection criteria used were top $\mathrm{K}$, subspace and variance. SPE scores were calculated as 
evaluation measurements. Specific experiments conducted for the thesis are described as follows.

\section{Experiment \#1: Pure data across all time ranges}

- Apply the profiling on $0 \%$ infection rate to each test sets.

- Run the modified PCA algorithm using a number of time shifts and selected component selection criteria. The number of time shifts varies from 1,2 and 3 . The selection criteria options are top K, subspace and variance.

- Average out the SPE scores from the ten test sets.

- Plot out average SPE scores versus time.

- Run for all combinations of time shifts and selection criteria.

- Repeat for all 16 time ranges.

\section{Experiment \#2: For each of the four dates in the long and low scenario}

- Apply the PCA profiling on the ten $20 \%$ infection rate test sets.

- Filter out any observations which are 15 times more than the mean on one date.

- Run the modified PCA algorithm using a number of time shifts and selected component selection criteria. The number of time shifts varies from 1,2 and 3. The selection criteria options are top $\mathrm{K}$, subspace and variance.

- Average out the SPE scores from the ten test sets.

- Apply each of the threshold methods to the average output.

- Apply the two evaluation techniques to each of the threshold methods.

- Run for all nine combinations of time shifts and selection criteria.

- Repeat using the ten $10 \%$, and the ten $5 \%$ infection rate test sets.

Experiment \#3 used the dates of the short and low scenario and repeated Experiment \#2.

Experiment \#4 used the dates of the long and high scenario, infection rates of $75 \%, 100 \%$ and $120 \%$ and repeated Experiment \#2. Experiment \#5 used the dates of the short and high scenario, infection rates of $75 \%, 100 \%$ and $120 \%$ and repeated Experiment $\# 2$. 


\subsubsection{Haar Wavelet Filtering Analysis Experiments}

A number of experiments with the Haar Wavelet Filtering were conducted. Three different data input sizes of 128, 256 and 512 and their respective filtering boundaries were used. In addition, the local variability weights of 5,1,0 and 3,2,0 where used. Local variability scores were calculated as evaluation measurements. Specific experiments conducted for the thesis are described as follows.

\section{Experiment \#1: Pure data across all dates}

- Apply the profiling using the different number of bins / bin width on $0 \%$ infection rate test sets. The number of bins per day is 128,256 and 512 .

- Run the Haar Wavelet algorithm on the three different profiles using the respective boundaries and weight combinations. See Table 5.4 for a list of sizes and boundaries. The weights (high pass, mid pass, low pass) used were $(5,1,0)$ and $(3,2,0)$

- Average out the Local Variability scores from the ten test sets.

- Plot out average Local Variability scores versus time.

- Run for all combinations of size/boundary and weightings.

\section{Experiment \#2: For each of the four time ranges in the long and low scenario}

- Apply the profiling using the different number of bins / bin width on $0 \%$ infection rate test sets. The number of bins per day is 128,256 and 512 .

- Run the Haar Wavelet algorithm on the three different profiles using the respective boundaries and weight combinations. Refer to Table 5.4.

- Average out the Local Variability scores from the ten test sets.

- Apply each of the threshold methods to the average output.

- Apply the two evaluation techniques to each of the threshold methods.

- Run for all 12 combinations of boundaries and weightings.

Experiment \#3 used the dates of the short and low scenario and repeated Experiment \#2.

Experiment \#4 used the dates of the long and high scenario, infection rates of $75 \%, 100 \%$ and 
$120 \%$ and repeated Experiment \#2. Experiment \#5 used the dates of the short and high scenario, infection rates of $75 \%, 100 \%$ and $120 \%$ and repeated Experiment $\# 2$.

\subsubsection{Hybrid PCA - Haar Wavelet Filtering Analysis Experiments}

\section{Experiment \#1: Pure data across all dates}

- Apply the PCA profiling on $0 \%$ infection rate test sets.

- Run the Hybrid algorithm on the created profile.

- Plot out average delta scores versus time.

- Repeat for each of the 16 time ranges.

\section{Experiment \#2: For each of the four dates in the long and low scenario}

- Apply the profiling to $20 \%$ infection rate test sets.

- Run the Hybrid algorithm on the created profile.

- Average out the delta scores from the ten test sets.

- Apply each of the threshold methods to the average output.

- Apply the two evaluation techniques to each of the threshold methods.

- Run for all 12 combinations of boundaries and weightings.

Experiment \#3 used the dates of the short and low scenario and repeated Experiment \#2. Experiment \#4 used the dates of the long and high scenario, infection rates of $75 \%, 100 \%$ and $120 \%$ and repeated Experiment \#2. Experiment \#5 used the dates of the short and high scenario, infection rates of $75 \%, 100 \%$ and $120 \%$ and repeated Experiment \#2. 


\subsection{Experimental Results and Discussion}

This chapter presents and discusses the results of the anomaly detection experiments conducted using the Kyoto2006+ data source. Experimental results and discussions for three different methods - Modified Principle Component Analysis, Wavelet Analysis, and the Hybrid approach - are presented in this chapter. Those three methods and the experimental procedure has been outlined in Chapter 5 .

\subsection{Evaluation of Modified Principle Component Analysis}

This section outlines the results obtained from running experiments with the Modified Principle Component Analysis (PCA) approach outlined in section 5.3.2 and concludes with a brief discussion.

\subsubsection{Modified PCA and Pure Data}

This section examines the results of the Modified PCA approach applied to a dataset with no infections. Figure 6.1 shows the SPE scores of January 6, 2009 on all ten test sets. This date is representative of the 16 selected dates. Even though there are ten test sets, there seem to be test sets which produce an extremely high score compared to the other observations. The value is so high that the other plots visually flatten to zero. Upon removing these test sets, as shown in Figure 6.2, all the remaining test sets behave alike. Examining the observations for the time bins with extremely high scores, it was observed that there were values 15 times larger than the particular feature mean. For the remaining experiments, the PCA data profiles were filtered prior to analysis. This filter removes time slices that have a feature value 15 times greater than the feature mean and flags them as potentially anomalous. Figure 6.3 shows the output of the 
Modified PCA approach applied to the filtered January 6, 2009 data. After applying the filter, all ten sets have a similar pattern. As a result, it is possible to create an average curve of the ten test sets which is representative of the entire date. This average is referred to as a footprint. Applying the filtering to the 16 dates, it is observed that each date has a distinct footprint. As shown in Figures 6.1, 6.2, and 6.3, regardless of the threshold technique, the algorithm suggests that there are anomalies. An anomaly is present if the score is a visually high value.

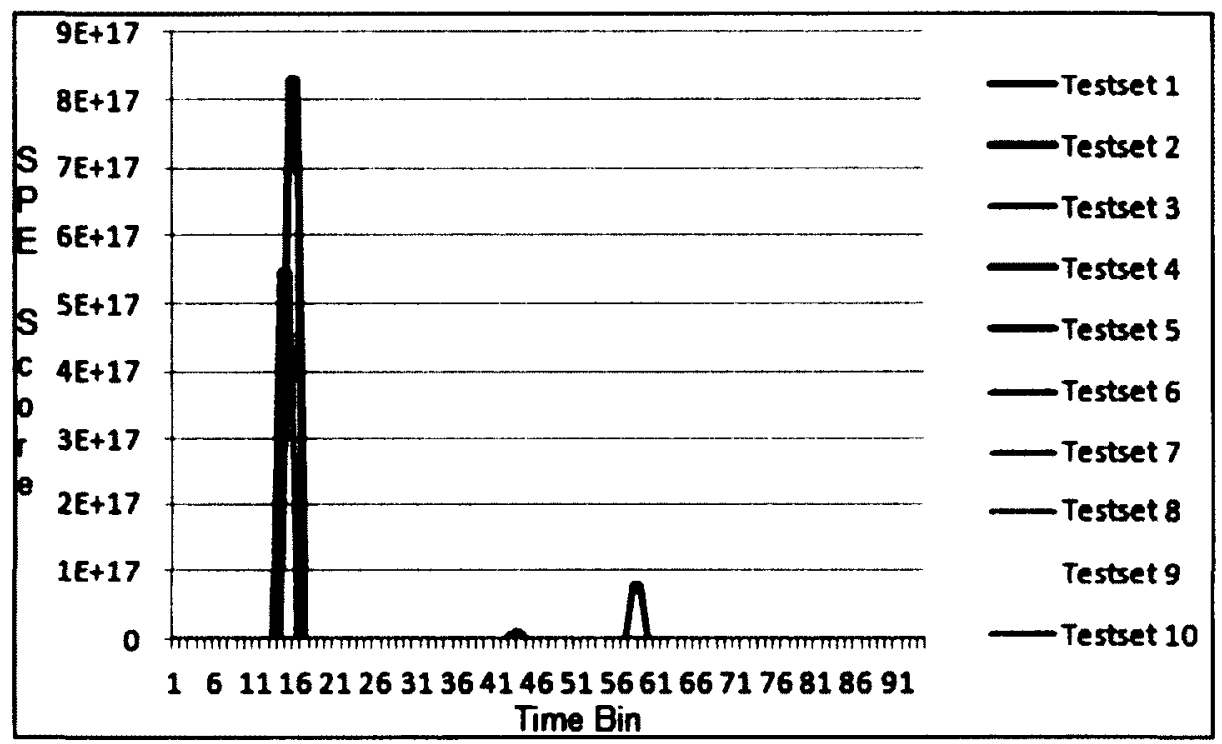

Figure 6.1: SPE scores from Unfiltered Modified PCA analysis on January 6, 2009 data

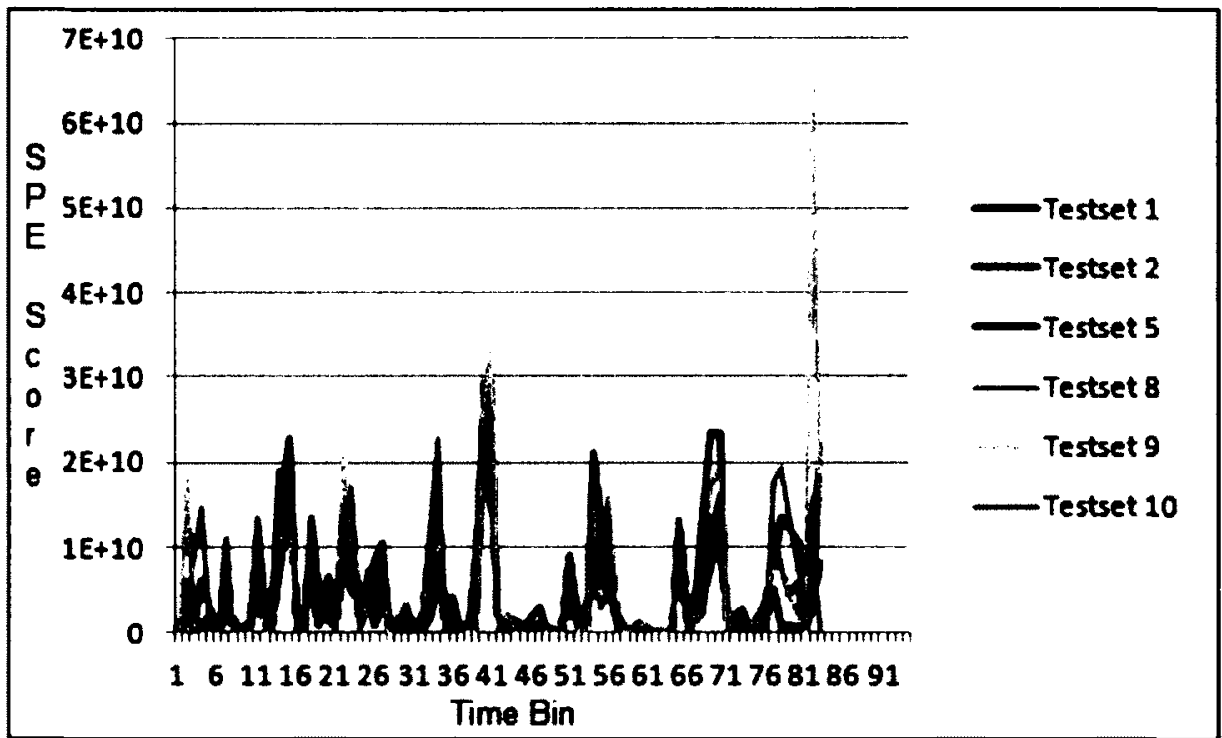

Figure 6.2: Subset of SPE scores from Modified PCA analysis on January 6, 2009 data 


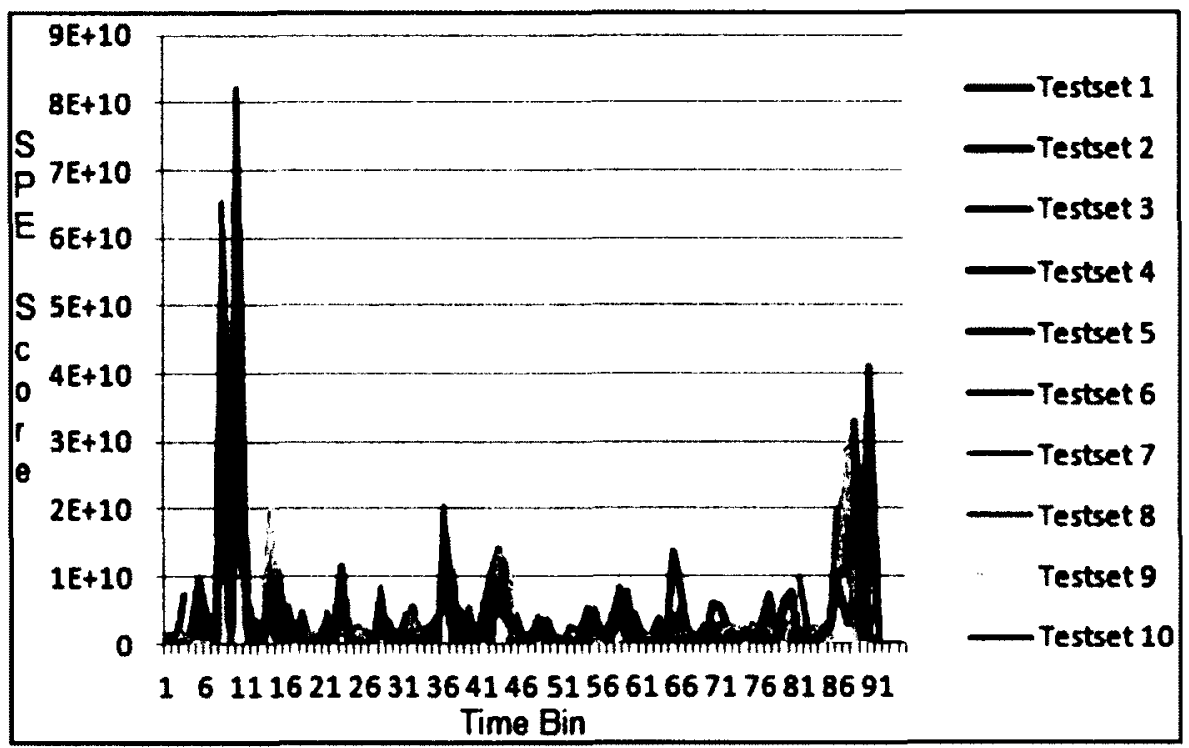

Figure 6.3: SPE scores from Filtered Modified PCA analysis on January 6, 2009 data

\subsubsection{Short and Low Infection}

This section examines the results of the Modified PCA experiments performed on a low infection rate in a short period of time. The four dates for this particular scenario, outlined in Table 5.2, all had consistent behaviour. As a result, for simplicity, only March 8, 2009 is discussed. Figure 6.4 illustrates the footprints observed with default parameters for the $5 \%, 10 \%$ and $20 \%$ infection ratio test sets (See Table 5.4 for default parameters). The infected time bins were bins 88,89 and 90 . None of these plots have a spike during the infected time bins, as these bins were filtered out, using the 15 times the column mean approach. Table 6.1 lists the hit/miss scores, and the hit/miss counts. The default threshold method generates a miss rate of about $15 \%$. For other threshold methods, the results obtained for miss count range from $25 \%$ to $35 \%$, see Section 6.1 .8 for details.

\begin{tabular}{|c|c|c|c|}
\hline Infection Rate & Score & Hit Count (\% Hit) & Miss Count (\% Miss) \\
\hline $5 \%$ & -2 & $83(86 \%)$ & $13(14 \%)$ \\
\hline $10 \%$ & -2 & $83(86 \%)$ & $13(14 \%)$ \\
\hline $20 \%$ & -2 & $84(87 \%)$ & $12(13 \%)$ \\
\hline
\end{tabular}

Table 6.1: March 8, 2009 - PCA experiment results 

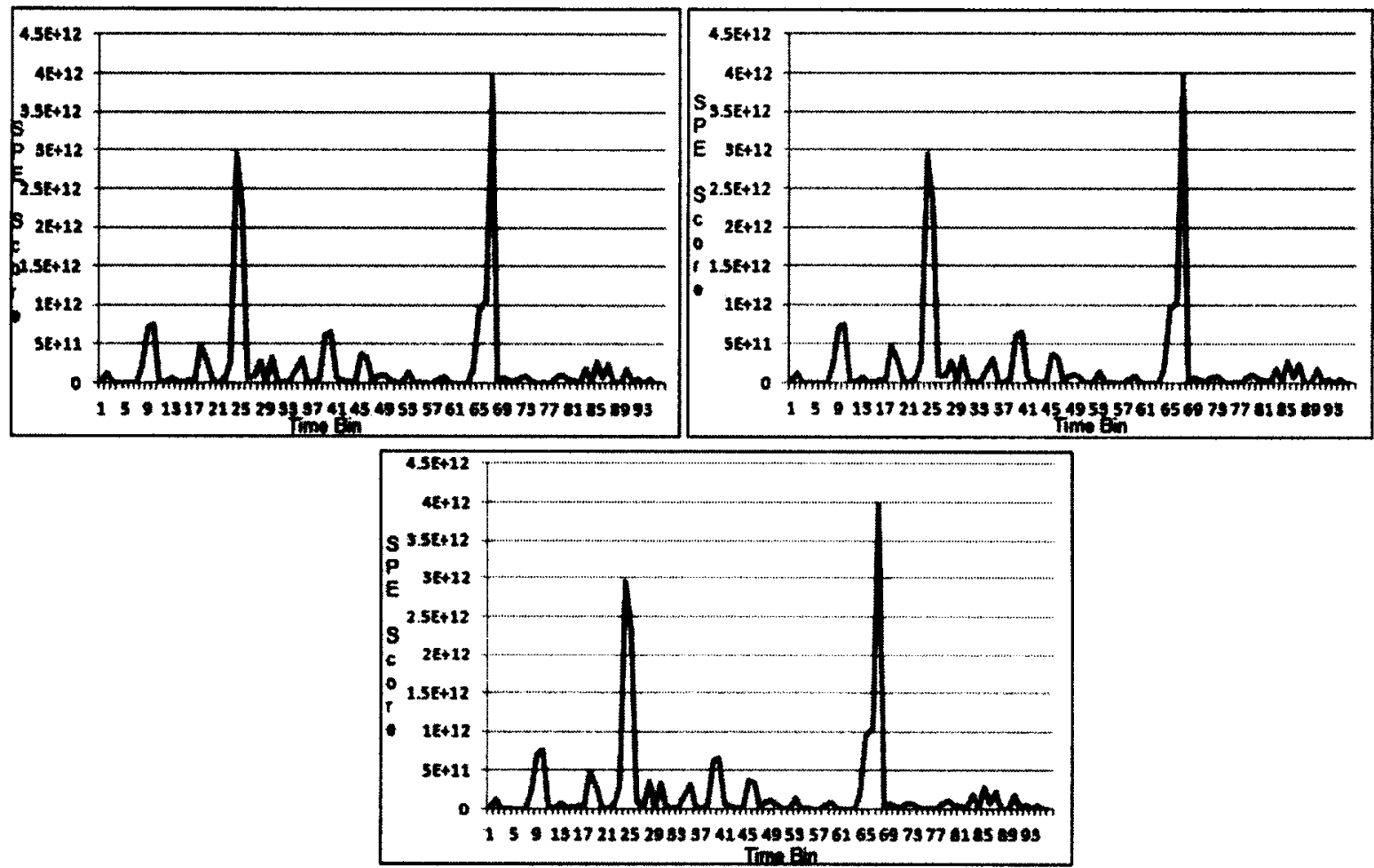

Figure 6.4: March 8, 2009 - PCA SPE scores 5\% (top left), 10\% (top right), 20\% (bottom) results

\subsubsection{Long and Low Infection}

This section examines the results of the Modified PCA approach experiments performed on a low infection rate in a long period of time. The four dates for this particular scenario, outlined in Table 5.2, all had consistent behaviour. As a result, for simplicity, only May 28, 2009 is discussed. Figure 6.5 illustrates the footprints observed under default parameters on the $5 \%, 10 \%$ and $20 \%$ infection ratio test sets. The infected time bins were bins $61-71$. As before, none of these plots had a spike during the infected time bins. A number of the time bins were filtered out. Table 6.2 lists the hit/miss scores and the hit/miss counts. The default threshold method generates a miss rate of about $20 \%$. The other threshold methods range from $25 \%$ to $40 \%$, see Section 6.1 .8 for details. 

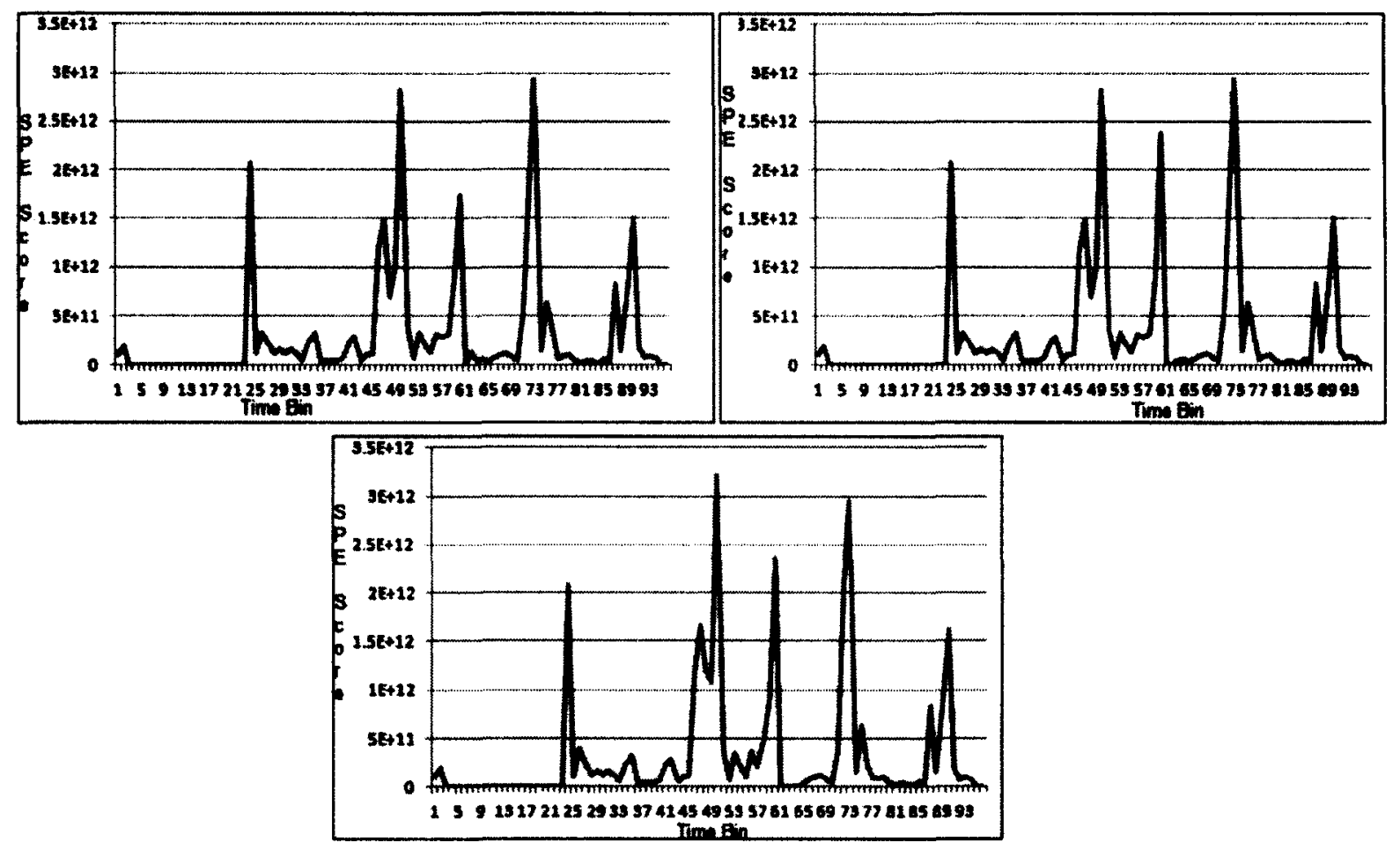

Figure 6.5: May 28, 2009 - PCA SPE scores 5\% (top left), 10\% (top right), 20\% (bottom) results

\begin{tabular}{|c|c|c|c|}
\hline Infection Rate & Score & Hit Count (\% Hit) & Miss Count (\% Miss) \\
\hline $5 \%$ & -2 & $75(78 \%)$ & $21(22 \%)$ \\
\hline $10 \%$ & -2 & $76(79 \%)$ & $20(21 \%)$ \\
\hline $20 \%$ & -2 & $77(80 \%)$ & $19(20 \%)$ \\
\hline
\end{tabular}

Table 6.2: May 28, 2009 - PCA experiment results

\subsubsection{Short and High Infection}

This section examines the results of the Modified PCA approach experiments performed on a high infection rate in a short period of time. The four dates for this particular scenario, outlined in Table 5.2, all had consistent behaviour. As a result, for simplicity, only January 15, 2009 is discussed. Figure 6.6 illustrates the footprints observed under default parameters on the $75 \%, 100 \%$ and $120 \%$ infection ratio test sets. The infected time bins were bins $83-86$. None of these plots had a spike during the infected time bins. These infected time bins were filtered out. Table 6.3 lists the hit/miss score and the hit/miss counts. The default threshold method 
generates a miss rate of about $30 \%$. The other threshold methods range from $35 \%$ to $40 \%$, see Section 6.1 .8 for details.
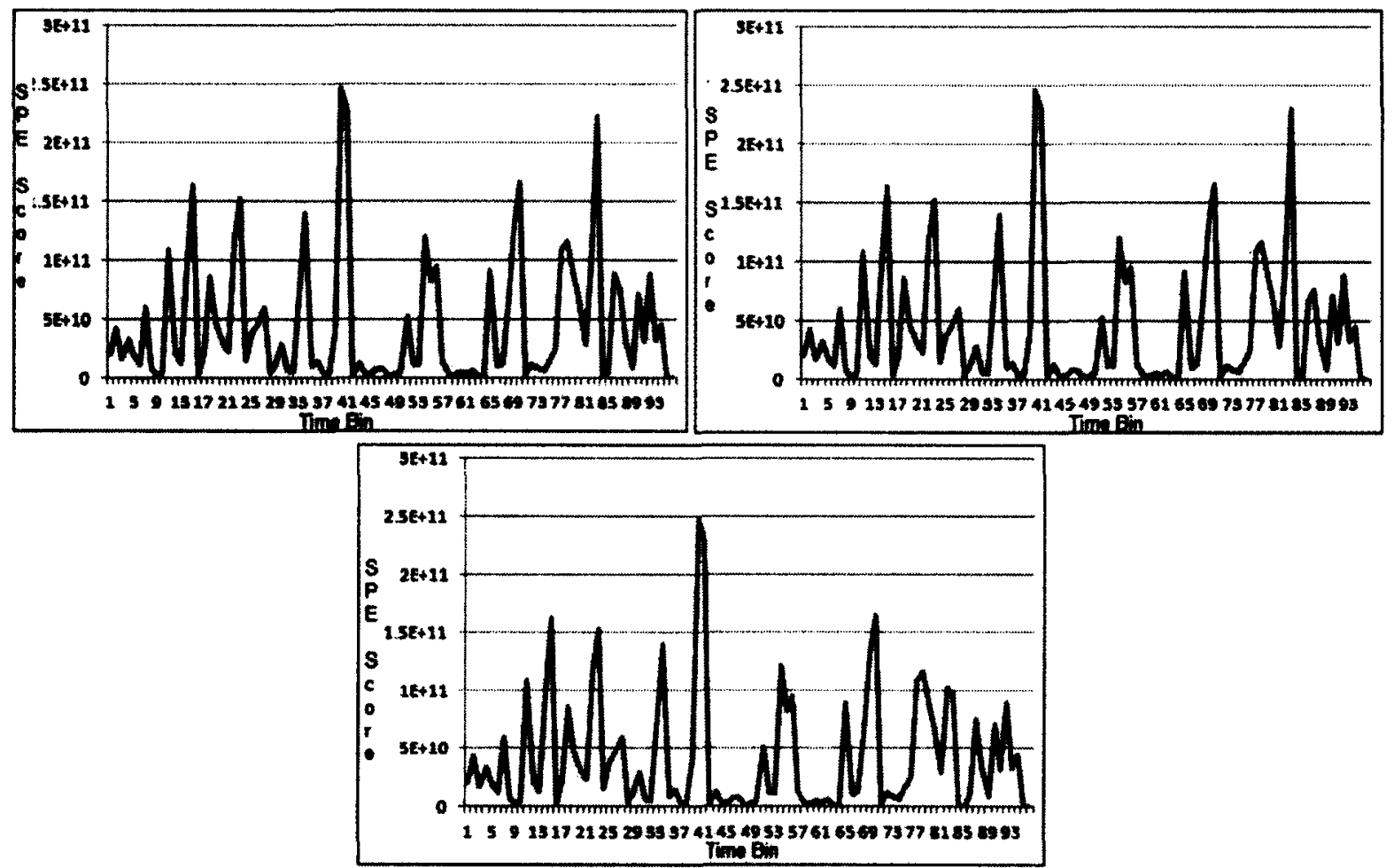

Figure 6.6: January 15, 2009 - PCA SPE scores 75\% (top left), 100\% (top right), 120\% (bottom) results

\begin{tabular}{|c|c|c|c|}
\hline Infection Rate & Score & Hit Count (\% Hit) & Miss Count (\% Miss) \\
\hline $75 \%$ & 0 & $66(69 \%)$ & $30(31 \%)$ \\
\hline $100 \%$ & 0 & $67(70 \%)$ & $29(30 \%)$ \\
\hline $120 \%$ & 0 & $66(69 \%)$ & $30(31 \%)$ \\
\hline
\end{tabular}

\subsubsection{Long and High Infection}

This section examines the results of the modified PCA algorithm experiments performed on a high infection rate in a long period of time. The four dates for this particular scenario, outlined in Table 5.2, all had consistent behaviour. As a result, for simplicity, only June 10, 2009 is discussed. Figure 6.7 illustrates the footprints observed under default parameters on the $75 \%, 100 \%$ and $120 \%$ infection ratio test sets. Unlike previous scenarios, the algorithm was able 
to determine the anomalous regions. Time bins $22-32$ are the infected time bins. Some of the time bins were filtered out, and a major spike highlighted the remainder. Table 6.4 outlines the hit/miss scores and the hit/miss counts measured using the default threshold. The threshold method generates a miss rate of about $20 \%$. The other threshold methods range from $15 \%$ to $35 \%$, see Section 6.1 .8 for details.
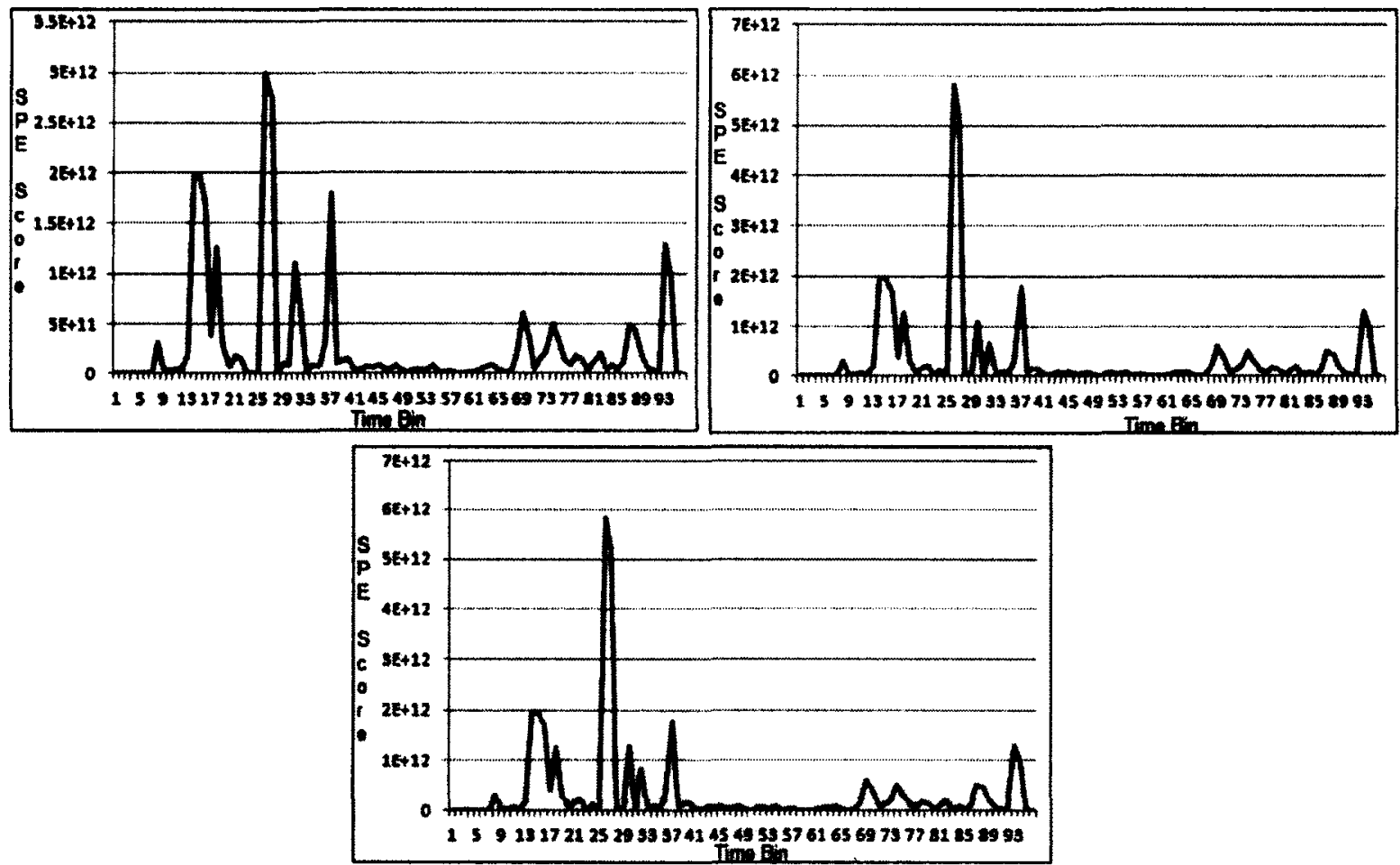

Figure 6.7: June 10, 2009 - PCA SPE scores 75\% (top left), 100\% (top right), 120\% (bottom) results

\begin{tabular}{|c|c|c|c|}
\hline Infection Rate & Score & Hit Count (\% Hit) & Miss Count (\% Miss) \\
\hline $75 \%$ & 0 & $76(79 \%)$ & $20(21 \%)$ \\
\hline $100 \%$ & 0 & $80(83 \%)$ & $16(17 \%)$ \\
\hline $120 \%$ & 0 & $78(81 \%)$ & $18(19 \%)$ \\
\hline
\end{tabular}

Table 6.4: June 10, 2009 - PCA experiment results

\subsubsection{Varying number of Time Shifts}

The time shifts were introduced by [6] in order to account for temporal change from [16]. Experiments were performed varying the number of time shifts and the results listed in tables 6.5 
- 6.8 summarize the measurements for each scenario on the specified dates. Increasing the number of time shifts never improved the score. The hit/miss scores either remained the same or decreased. Increasing the time shifts increased the miss count. This direct relationship showed that, using the Kyoto2006+ dataset, one time shift was optimal.

\begin{tabular}{|c|c|c|c|c|}
\hline Infection Rate & Time Shifts & Score & Hit Count & Miss Count \\
\hline \multirow{3}{*}{$5 \%$} & 1 & -2 & 83 & 13 \\
\cline { 2 - 5 } & 2 & -2 & 78 & 18 \\
\cline { 2 - 5 } & 3 & -6 & 73 & 23 \\
\hline \multirow{3}{*}{$10 \%$} & 1 & -2 & 83 & 13 \\
\cline { 2 - 5 } & 2 & -2 & 78 & 18 \\
\cline { 2 - 5 } & 3 & -6 & 74 & 22 \\
\hline \multirow{3}{*}{$20 \%$} & 1 & -2 & 84 & 12 \\
\cline { 2 - 5 } & 2 & -2 & 78 & 18 \\
\cline { 2 - 5 } & 3 & -6 & 71 & 25 \\
\hline
\end{tabular}

Table 6.5: March 8, 2009 - PCA Short and Low infection varying time shift results

\begin{tabular}{|c|c|c|c|c|}
\hline Infection Rate & Time Shifts & Score & Hit Count & Miss Count \\
\hline \multirow{3}{*}{$5 \%$} & 1 & -2 & 75 & 21 \\
\cline { 2 - 5 } & 2 & -4 & 74 & 22 \\
\hline \multirow{3}{*}{$10 \%$} & 3 & -6 & 71 & 25 \\
\cline { 2 - 5 } & 1 & -2 & 76 & 20 \\
\cline { 2 - 5 } & 2 & -4 & 73 & 23 \\
\hline \multirow{3}{*}{$20 \%$} & 3 & -6 & 71 & 25 \\
\cline { 2 - 5 } & 1 & -2 & 77 & 19 \\
\cline { 2 - 5 } & 2 & -4 & 75 & 21 \\
\hline
\end{tabular}

Table 6.6: May 28, 2009 - PCA Long and Low infection varying time shift results

\begin{tabular}{|c|c|c|c|c|}
\hline Infection Rate & Time Shifts & Score & Hit Count & Miss Count \\
\hline \multirow{3}{*}{$75 \%$} & 1 & 0 & 66 & 30 \\
\cline { 2 - 5 } & 2 & 0 & 63 & 33 \\
\cline { 2 - 5 } & 3 & 0 & 60 & 36 \\
\hline \multirow{3}{*}{$100 \%$} & 1 & 0 & 67 & 29 \\
\cline { 2 - 5 } & 2 & 0 & 64 & 32 \\
\cline { 2 - 5 } & 3 & 0 & 63 & 33 \\
\cline { 2 - 5 } & 1 & 0 & 66 & 30 \\
\cline { 2 - 5 } & 2 & 0 & 67 & 29 \\
\hline
\end{tabular}

Table 6.7: January 15, 2009 - PCA Short and High infection varying time shift results 


\begin{tabular}{|c|c|c|c|c|}
\hline Infection Rate & Time Shifts & Score & Hit Count & Miss Count \\
\hline \multirow{3}{*}{$75 \%$} & 1 & 0 & 76 & 20 \\
\cline { 2 - 5 } & 2 & -1 & 65 & 31 \\
\cline { 2 - 5 } & 3 & -3 & 60 & 36 \\
\hline \multirow{3}{*}{$100 \%$} & 1 & 0 & 80 & 16 \\
\cline { 2 - 5 } & 2 & -1 & 81 & 15 \\
\cline { 2 - 5 } & 3 & -3 & 66 & 33 \\
\cline { 2 - 5 } & 1 & 0 & 78 & 18 \\
\cline { 2 - 5 } & 2 & -1 & 79 & 33 \\
\hline
\end{tabular}

Table 6.8: June 10, 2009 - PCA Long and High infection varying time shift results

\subsubsection{Varying Selection Criteria}

The selection criterion determines what and how many components are used in analysis. Experiments varying the selection criterion between three options were performed. As outlined in section 2.4 , the subspace approach, the variance approach and the top one approach were used. The results listed in tables $6.9-6.12$ summarize the evaluation measurements for selection criteria on the specified dates. Selecting any component with a variance of one or greater performed the worst. It was observed that this approach consistently had the worse hit/miss score and hit/miss count. Selecting only the top component performed better than the variance approach in most cases; however, the subspace approach performed the best in all cases.

\begin{tabular}{|c|c|c|c|c|}
\hline $\begin{array}{c}\text { Infection } \\
\text { Rate }\end{array}$ & Measurement & $\begin{array}{c}\text { Subspace } \\
\text { selection }\end{array}$ & $\begin{array}{c}\text { Variance }>1 \\
\text { selection }\end{array}$ & $\begin{array}{c}\text { Top K = 1 } \\
\text { components }\end{array}$ \\
\hline \multirow{3}{*}{$5 \%$} & Score & -2 & -2 & -2 \\
\cline { 2 - 5 } & Hit Count & 83 & 59 & 83 \\
\cline { 2 - 5 } & Miss Count & 13 & 37 & 13 \\
\hline \multirow{3}{*}{$10 \%$} & Score & -2 & -2 & -2 \\
\cline { 2 - 5 } & Hit Count & 83 & 58 & 83 \\
\cline { 2 - 5 } & Miss Count & 13 & 38 & 13 \\
\hline \multirow{2}{*}{$20 \%$} & Score & -2 & -2 & -2 \\
\cline { 2 - 5 } & Hit Count & 84 & 57 & 84 \\
\cline { 2 - 5 } & Miss Count & 12 & 39 & 12 \\
\hline
\end{tabular}

Table 6.9: March 8, 2009 - PCA Short and Low infection varying selection criteria results 


\begin{tabular}{|c|c|c|c|c|}
\hline $\begin{array}{c}\text { Infection } \\
\text { Rate }\end{array}$ & Measurement & $\begin{array}{c}\text { Subspace } \\
\text { selection }\end{array}$ & $\begin{array}{c}\text { Variance }>1 \\
\text { selection }\end{array}$ & $\begin{array}{c}\text { Top K=1 } \\
\text { components }\end{array}$ \\
\hline \multirow{3}{*}{$5 \%$} & Score & -2 & -2 & -2 \\
\cline { 2 - 5 } & Hit Count & 75 & 61 & 75 \\
\cline { 2 - 5 } & Miss Count & 21 & 35 & 21 \\
\hline \multirow{2}{*}{$10 \%$} & Score & -2 & -2 & -2 \\
\cline { 2 - 5 } & Hit Count & 76 & 63 & 76 \\
\cline { 2 - 5 } & Miss Count & 20 & 33 & 20 \\
\hline \multirow{2}{*}{$20 \%$} & Score & -2 & -2 & -2 \\
\cline { 2 - 5 } & Hit Count & 77 & 60 & 17 \\
\cline { 2 - 5 } & Miss Count & 19 & 36 & 19 \\
\hline
\end{tabular}

Table 6.10: May 28, 2009 - PCA Long and Low infection varying selection criteria results

\begin{tabular}{|c|c|c|c|c|}
\hline $\begin{array}{c}\text { Infection } \\
\text { Rate }\end{array}$ & Measurement & $\begin{array}{c}\text { Subspace } \\
\text { selection }\end{array}$ & $\begin{array}{c}\text { Variance }>\text { 1 } \\
\text { selection }\end{array}$ & $\begin{array}{c}\text { Top K=1 } \\
\text { components }\end{array}$ \\
\hline \multirow{3}{*}{$75 \%$} & Score & 0 & 0 & 0 \\
\cline { 2 - 5 } & Hit Count & 66 & 55 & 67 \\
\cline { 2 - 5 } & Miss Count & 30 & 41 & 29 \\
\hline \multirow{2}{*}{$100 \%$} & Score & 0 & 1 & 0 \\
\cline { 2 - 5 } & Hit Count & 67 & 55 & 68 \\
\cline { 2 - 5 } & Miss Count & 29 & 41 & 28 \\
\hline \multirow{2}{*}{$120 \%$} & Score & 0 & 0 & 67 \\
\cline { 2 - 5 } & Hit Count & 66 & 57 & 29 \\
\cline { 2 - 5 } & Miss Count & 30 & 39 & 67 \\
\hline
\end{tabular}

Table 6.11: January 15, 2009 - PCA Short and High infection varying selection criteria results

\begin{tabular}{|c|c|c|c|c|}
\hline $\begin{array}{c}\text { Infection } \\
\text { Rate }\end{array}$ & Measurement & $\begin{array}{c}\text { Subspace } \\
\text { selection }\end{array}$ & $\begin{array}{c}\text { Variance }>1 \\
\text { selection }\end{array}$ & $\begin{array}{c}\text { Top K=1 } \\
\text { components }\end{array}$ \\
\hline \multirow{3}{*}{$75 \%$} & Score & 0 & 2 & 0 \\
\cline { 2 - 5 } & Hit Count & 76 & 64 & 76 \\
\cline { 2 - 5 } & Miss Count & 20 & 32 & 20 \\
\hline \multirow{2}{*}{$100 \%$} & Score & 0 & 3 & 0 \\
\cline { 2 - 5 } & Hit Count & 80 & 67 & 79 \\
\cline { 2 - 5 } & Miss Count & 16 & 29 & 17 \\
\hline \multirow{2}{*}{$120 \%$} & Score & 0 & 4 & 78 \\
\cline { 2 - 5 } & Hit Count & 78 & 68 & 18 \\
\cline { 2 - 5 } & Miss Count & 18 & 28 & 0 \\
\hline
\end{tabular}

Table 6.12: June 10, 2009 - PCA Long and High infection varying selection criteria results 


\subsubsection{Threshold Techniques and Modified PCA}

Each of the experiments performed used four different threshold techniques to automatically identify anomalous time bins. The results listed in tables $6.13-6.16$ summarize the evaluation measurements for threshold techniques on the specified dates. The weighted average and moving average consistently had a lower SPE score and higher miss count. The moving average without outliers performed the best in scenarios with low infection rates. In scenarios with higher infection rates, the static average performed slightly better than the moving average without outliers.

\begin{tabular}{|c|c|c|c|c|c|}
\hline $\begin{array}{c}\text { Infection } \\
\text { Rate }\end{array}$ & Measurement & $\begin{array}{c}\text { Static } \\
\text { Average }\end{array}$ & $\begin{array}{c}\text { Moving } \\
\text { Average }\end{array}$ & $\begin{array}{c}\text { Moving } \\
\text { Average } \\
\text { without outliers }\end{array}$ & $\begin{array}{c}\text { Weighted } \\
\text { Average }\end{array}$ \\
\hline \multirow{3}{*}{$5 \%$} & Score & -2 & -2 & -2 & -2 \\
\cline { 2 - 6 } & Hit Count & 76 & 68 & 83 & 74 \\
\cline { 2 - 6 } & Miss Count & 20 & 28 & 13 & 22 \\
\hline \multirow{2}{*}{$10 \%$} & Score & -2 & -2 & -2 & -2 \\
\cline { 2 - 6 } & Hit Count & 76 & 68 & 83 & 74 \\
\cline { 2 - 6 } & Miss Count & 20 & 28 & 13 & 22 \\
\hline \multirow{2}{*}{$20 \%$} & Score & -2 & -2 & -2 & -2 \\
\cline { 2 - 6 } & Hit Count & 76 & 68 & 84 & 74 \\
\cline { 2 - 6 } & Miss Count & 20 & 28 & 12 & 22 \\
\hline
\end{tabular}

Table 6.13: March, 8, 2009 - PCA Short and Low threshold results

\begin{tabular}{|c|c|c|c|c|c|}
\hline $\begin{array}{c}\text { Infection } \\
\text { Rate }\end{array}$ & Measurement & $\begin{array}{c}\text { Static } \\
\text { Average }\end{array}$ & $\begin{array}{c}\text { Moving } \\
\text { Average }\end{array}$ & $\begin{array}{c}\text { Moving } \\
\text { Average } \\
\text { without outliers }\end{array}$ & $\begin{array}{c}\text { Weighted } \\
\text { Average }\end{array}$ \\
\hline \multirow{3}{*}{$5 \%$} & Score & -2 & -26 & -2 & -2 \\
\cline { 2 - 6 } & Hit Count & 74 & 62 & 75 & 75 \\
\cline { 2 - 6 } & Miss Count & 22 & 34 & 21 & 21 \\
\hline \multirow{2}{*}{$10 \%$} & Score & -2 & -26 & -2 & -2 \\
\cline { 2 - 6 } & Hit Count & 74 & 62 & 76 & 75 \\
\cline { 2 - 6 } & Miss Count & 22 & 34 & 20 & 21 \\
\hline \multirow{2}{*}{$20 \%$} & Score & -2 & -26 & -2 & -2 \\
\cline { 2 - 6 } & Hit Count & 75 & 61 & 77 & 76 \\
\cline { 2 - 6 } & Miss Count & 21 & 35 & 19 & 20 \\
\hline
\end{tabular}

Table 6.14: May 28, 2009 - PCA Long and Low threshold results 


\begin{tabular}{|c|c|c|c|c|c|}
\hline $\begin{array}{c}\text { Infection } \\
\text { Rate }\end{array}$ & Measurement & $\begin{array}{c}\text { Static } \\
\text { Average }\end{array}$ & $\begin{array}{c}\text { Moving } \\
\text { Average }\end{array}$ & $\begin{array}{c}\text { Moving } \\
\text { Average } \\
\text { without outliers }\end{array}$ & $\begin{array}{c}\text { Weighted } \\
\text { Average }\end{array}$ \\
\hline \multirow{3}{*}{$75 \%$} & Score & 0 & 0 & 0 & 0 \\
\cline { 2 - 6 } & Hit Count & 64 & 63 & 66 & 61 \\
\cline { 2 - 6 } & Miss Count & 32 & 33 & 30 & 35 \\
\hline \multirow{2}{*}{$100 \%$} & Score & 1 & 0 & 0 & 1 \\
\cline { 2 - 6 } & Hit Count & 64 & 64 & 67 & 62 \\
\cline { 2 - 6 } & Miss Count & 32 & 32 & 29 & 34 \\
\hline \multirow{3}{*}{$120 \%$} & Score & 0 & 0 & 0 & 0 \\
\cline { 2 - 6 } & Hit Count & 63 & 62 & 66 & 63 \\
\cline { 2 - 6 } & Miss Count & 33 & 34 & 30 & 33 \\
\hline
\end{tabular}

Table 6.15: January 15, 2009 - PCA Short and High threshold results

\begin{tabular}{|c|c|c|c|c|c|}
\hline $\begin{array}{c}\text { Infection } \\
\text { Rate }\end{array}$ & Measurement & $\begin{array}{c}\text { Static } \\
\text { Average }\end{array}$ & $\begin{array}{c}\text { Moving } \\
\text { Average }\end{array}$ & $\begin{array}{c}\text { Moving } \\
\text { Average } \\
\text { without outliers }\end{array}$ & $\begin{array}{c}\text { Weighted } \\
\text { Average }\end{array}$ \\
\hline \multirow{3}{*}{$75 \%$} & Score & 1 & 1 & 0 & 1 \\
\cline { 2 - 6 } & Hit Count & 76 & 66 & 76 & 71 \\
\cline { 2 - 6 } & Miss Count & 20 & 30 & 20 & 25 \\
\hline \multirow{3}{*}{$100 \%$} & Score & 3 & 0 & 0 & 1 \\
\cline { 2 - 6 } & Hit Count & 80 & 66 & 80 & 72 \\
\cline { 2 - 6 } & Miss Count & 16 & 30 & 16 & 24 \\
\hline \multirow{3}{*}{$120 \%$} & Score & 4 & 1 & 0 & 1 \\
\cline { 2 - 6 } & Hit Count & 81 & 66 & 78 & 72 \\
\cline { 2 - 6 } & Miss Count & 15 & 30 & 18 & 24 \\
\hline
\end{tabular}

Table 6.16: June 10, 2009 - PCA Long and High threshold results

\subsubsection{Summary and Discussion}

The major advantage of the Modified Principle Component Analysis (PCA) approach was that it considered all of the features at once. Regardless of how many features, all were considered in analysis and a single score was calculated for each time bin. There were two user defined parameters: the number of time shifts and the component selection criteria.

The number of time shifts. In [6], the authors showed that four or more time shifts do not have any significant impact. Our research examined one, two and three time shifts. The number of time shifts affects how much the algorithm considers changes in time. For instance, a large 
number time shifts will show a great deal of change in a feature, and changes will be very common. The algorithm will become less sensitive to change [6]. Conversely, no time shifts fail to take into account any the temporal change. There is a need for modest consideration of how the data changes over the next few observations. Our analysis concluded that one time shift performed the best. This suggests that there is a great deal of change between time bins in the Kyoto2006+ and re-enforces the need for considering temporal changes. As more time shifts were applied, the algorithm was less sensitive to the change and performed worse.

Component Selection Criteria. The Modified PCA approach determines a set of ranked components and only a subset of are used in anomaly detection. The number of selected components affects subspace creation and the SPE score calculation. A selection of a large number of components converges close to the original feature space. It would not unlock and new patterns or provide an optimizations. Overly simple components would miss out on important information and not be very meaningful. Ideally, a handful of independent components are needed to form the anomalous space. In our analysis, it was observed that in most cases, the Top $K=1$ approach and the subspace approach had the same results. This suggests the subspace approach used one, or close to one, components to build the subspace. It can be concluded that there are a number of redundant features in the Kyoto2006+ dataset. A few components describe the entire dataset. Considering all features with variance greater than one builds involves a larger number of components, and explains why this approach performed the worst.

A number of test sets were built using different days in the week, and different infection ratios. Regardless of the infection rate or day in week, the output SPE scores of the modified PCA algorithm highlighted time bins. The output SPE score was the Euclidean Magnitude, and 
is directly related to the size of the projected. The SPE score were always large and is due to the nature of the observations, and the measurement. This can be illustrated in the $0 \%$ infection rate experiments. The algorithm considered all features at once and was able to modestly pick out the infected time bins. It performed best in a situation where there are a lot of malicious flows over a long period of time.

\subsection{Evaluation of Haar Wavelet Analysis}

This section outlines the results obtained from running the experiments with the Haar Wavelet Analysis approach outlined in section 5.3.3 and concludes with a brief discussion.

\subsubsection{Haar Wavelet Analysis and Pure data}

This section examines the results of the Haar wavelet filtering analysis applied to a dataset with no infections. The experiments performed in this section examine all infection scenarios. Figure 6.8 shows the local variability scores for each TCP feature and Figure 6.9 shows local variability scores for each OTHER feature of all test sets on January 6, 2009. This date is representative of the 16 selected dates. It can be observed that, for a few features, there exists a test set with much higher local variability values than the rest of the test sets. Similar to the Modified PCA approach, it is determined that an observation 15 times larger than the mean of that feature. Upon removing the test sets, a footprint was noticed across the remaining test sets. Unlike the Modified PCA approach, the local variability score did not have an extreme difference in values and did not flatten the rest of the plot to zero. Also, the average calculations are not affected. The Haar Wavelet Filtering analysis does not use initial filtering. The average

local variability is used in analysis and is referred to as the footprint. As shown in Figure 6.10 
and Figure 6.11, regardless of the threshold technique, the algorithm will suggest that there are anomalies present.
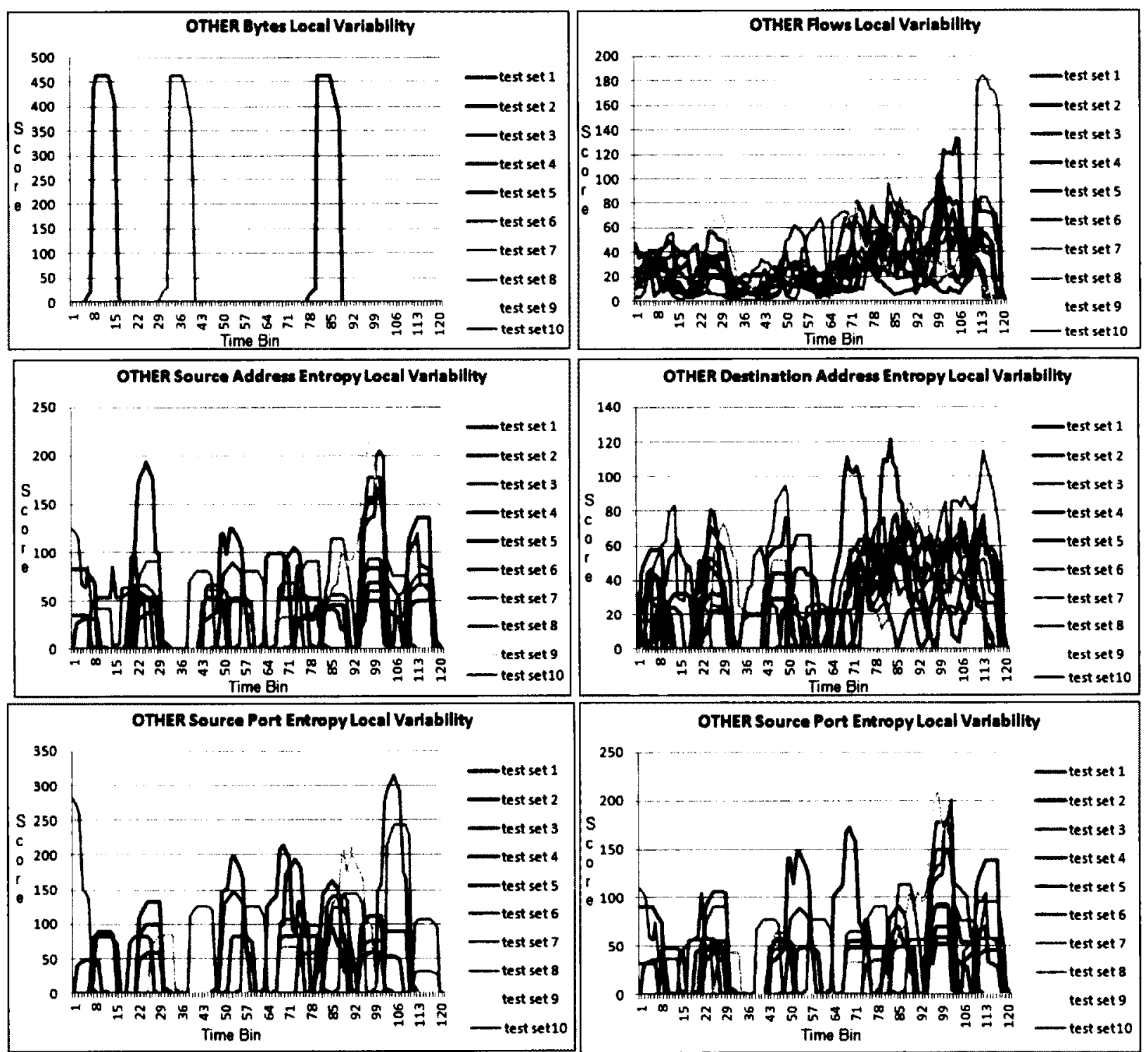

Figure 6.8: January 6, 2009 - OTHER Wavelet flows Local Variability Scores 


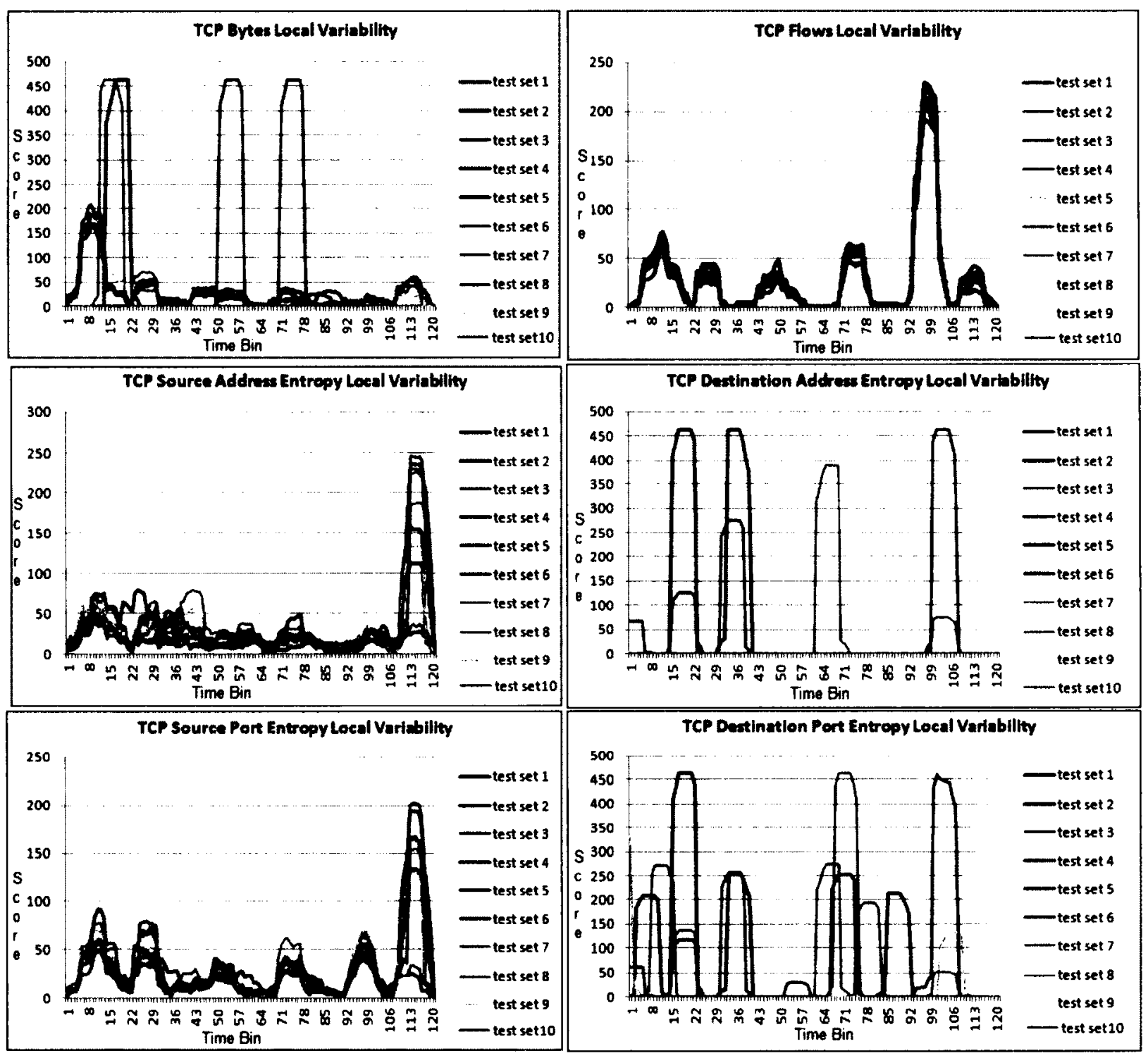

Figure 6.9: January 6, 2009 - TCP Wavelet flows Local Variability Scores 

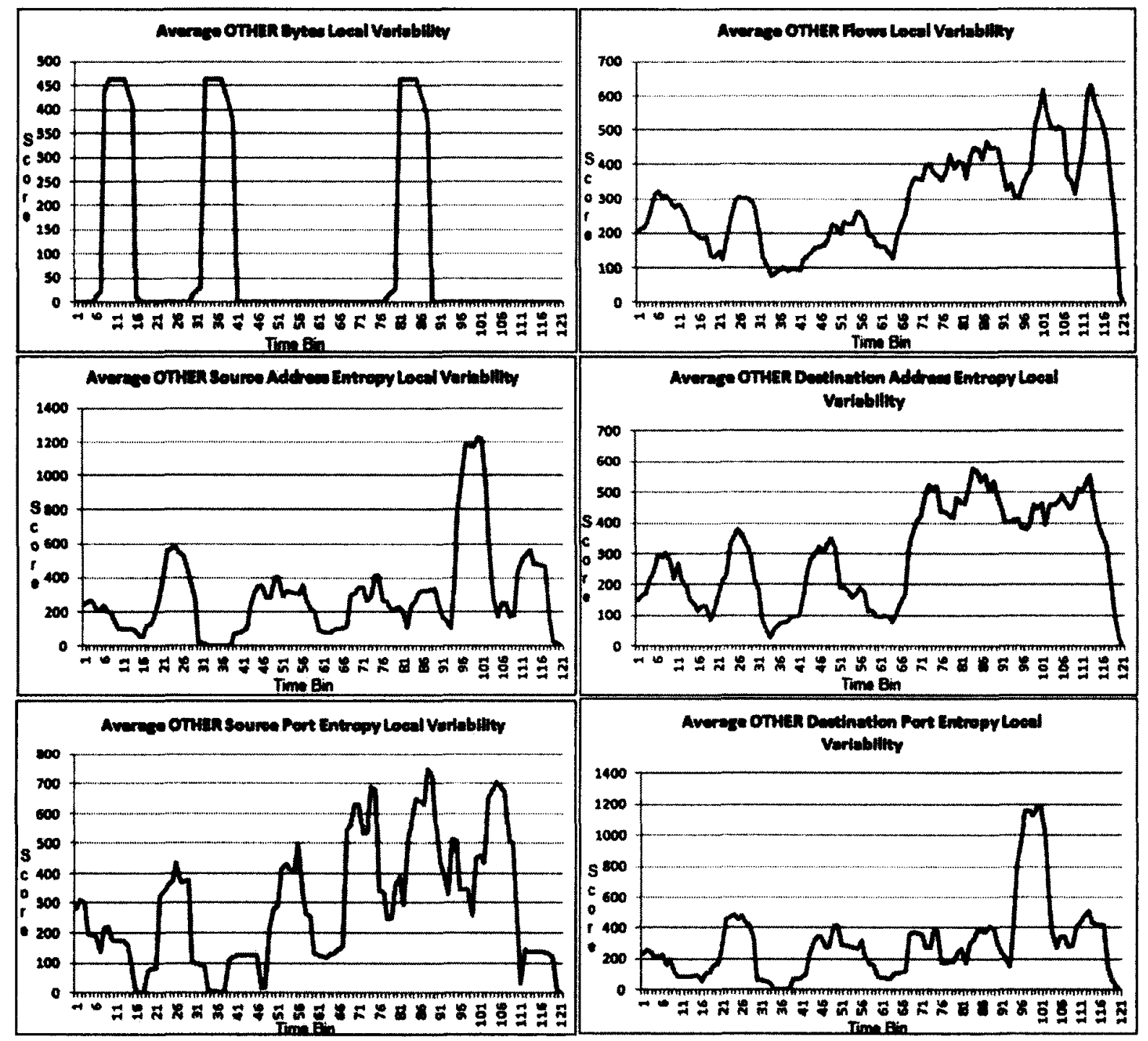

Figure 6.10: January 6, 2009 - Average OTHER Wavelet flows Local Variability Scores 

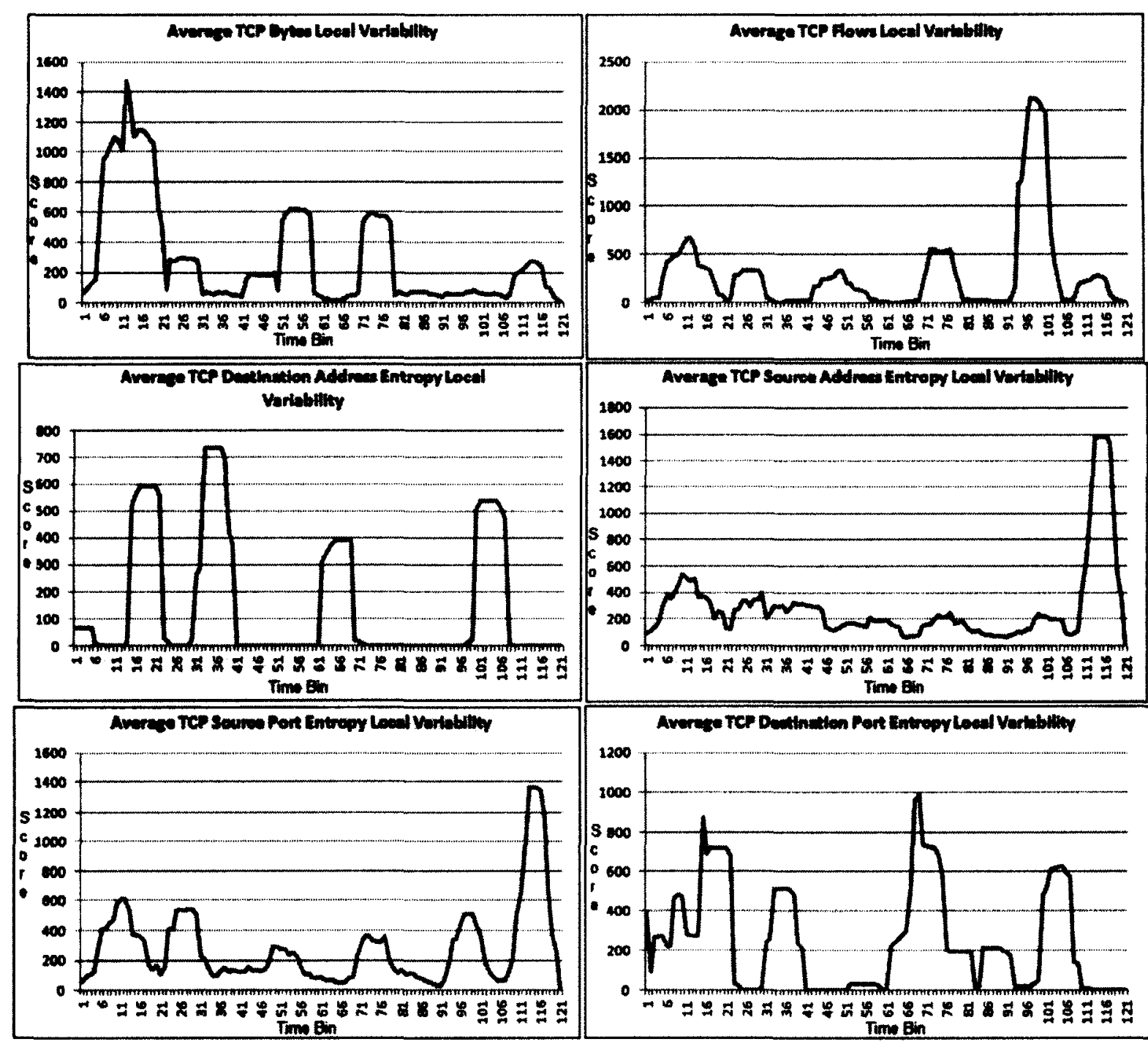

Figure 6.11: January 6, 2009 - Average TCP Wavelet flows Local Variability Scores

\subsubsection{Short and Low Infection}

This section examines the results of the Haar Wavelet experiments performed on a low infection rate in a short period of time. The four dates for this particular scenario, outlined in Table 5.2, all had consistent behaviour. As a result, for simplicity, only March 8, 2009 is discussed. Figure 6.12 and Figure 6.13 illustrate the footprints observed with default parameters for the TCP and OTHER features for the $5 \%$ infection ratio (See Table 5.4 for a list of default 
conditions). The experiments were conducted on test sets of $5 \%, 10 \%$ and $20 \%$ infection ratios. The infected time bins were bins 116,117 and 118. A number of the feature plots depicted spikes during the infected times. Table 6.17 lists the hit/miss scores and the hit/miss counts measured for each feature. The default threshold method determines a $19 \%$ miss rate in the best feature and about a $64 \%$ miss rate in the worst performing feature. The other threshold methods range from $5 \%$ to $65 \%$, see Section 6.2 .8 for details.
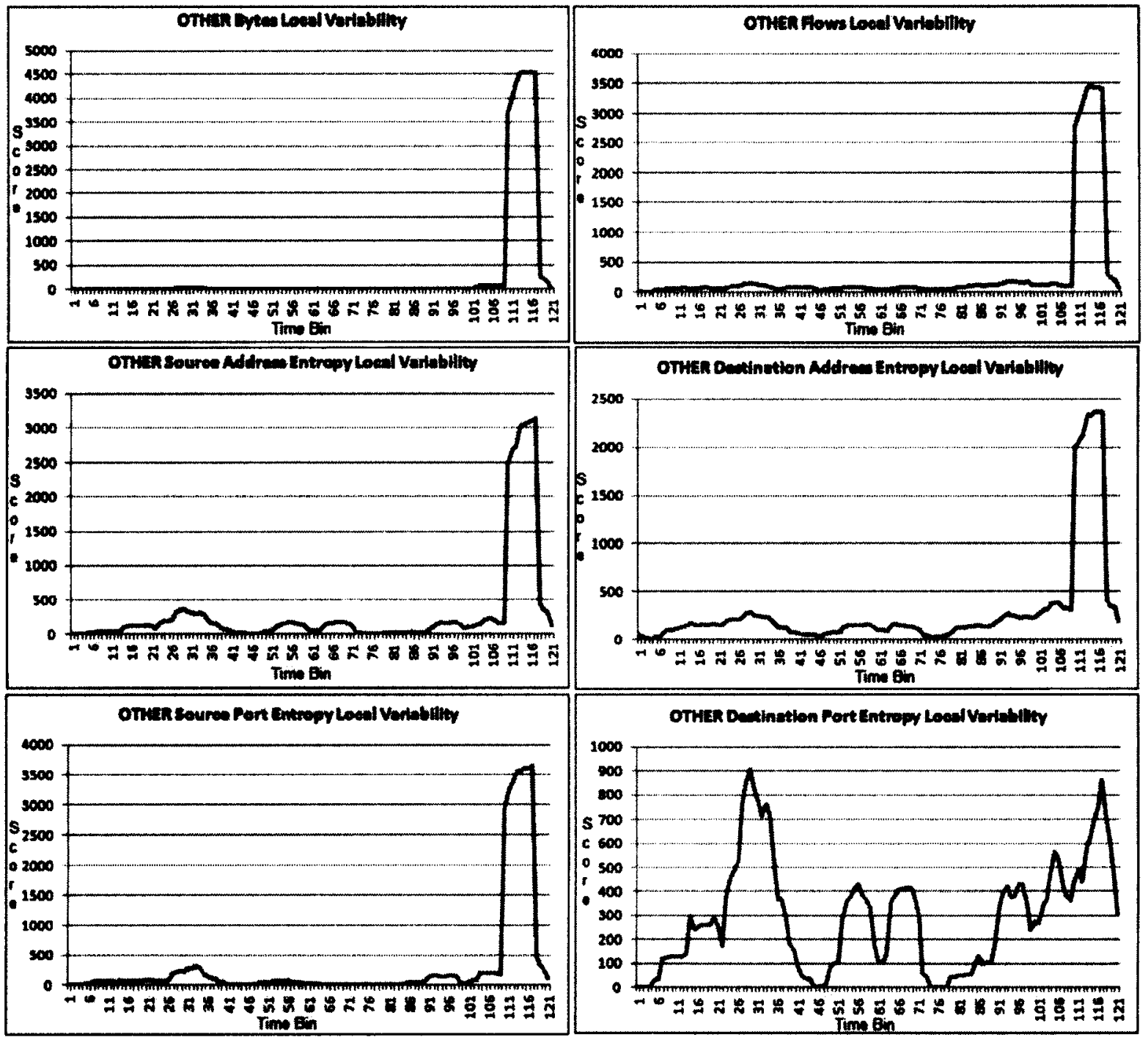

Figure 6.12: March 8, 2009 - Average OTHER Wavelet Local Variability Scores with 5\% infection rate 

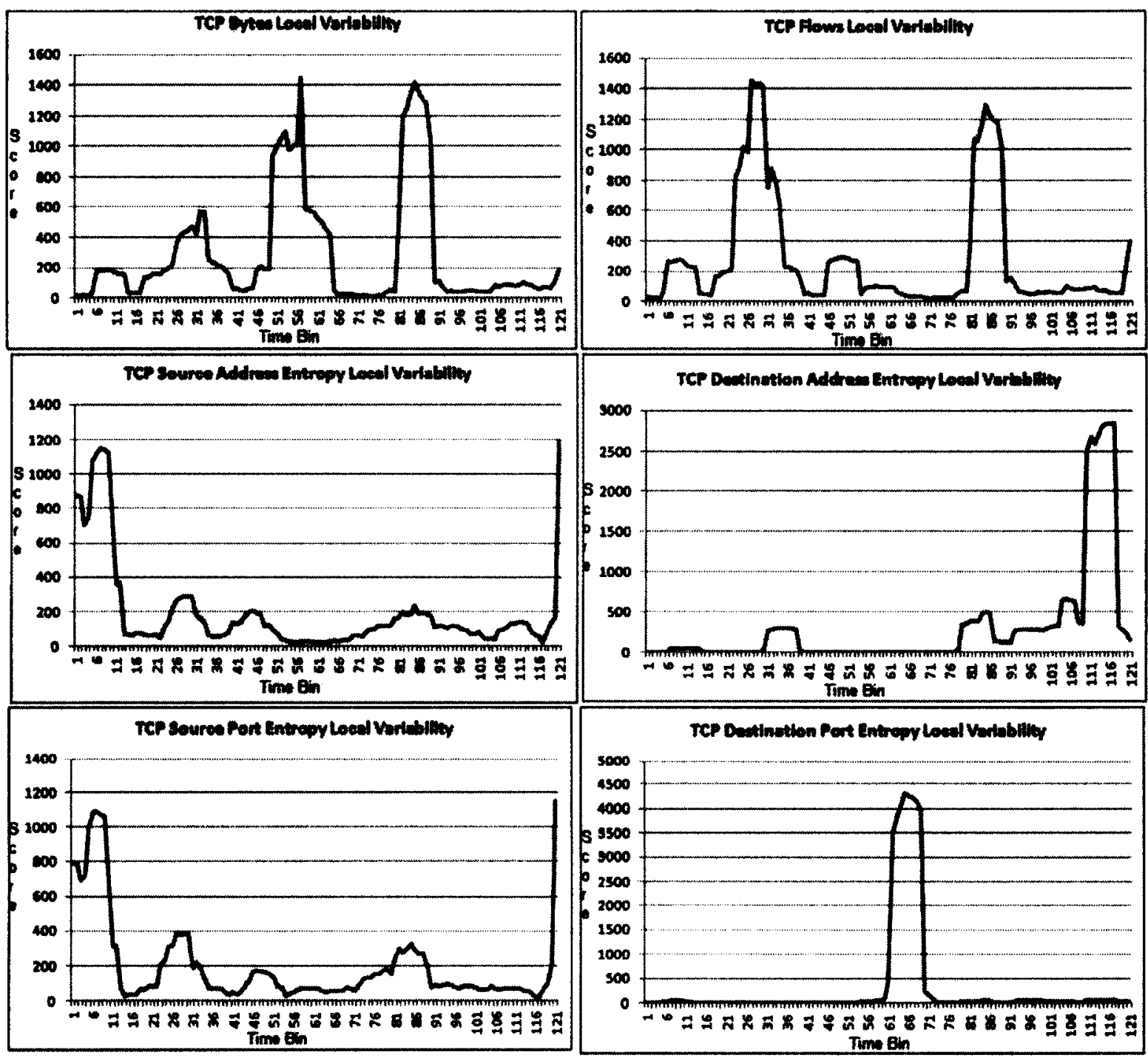

Figure 6.13: March 8, 2009 - Average TCP Wavelet Local Variability Scores with 5\% infection rate 


\begin{tabular}{|c|c|c|c|c|}
\hline $\begin{array}{c}\text { Infection } \\
\text { Rate }\end{array}$ & Feature & Score & $\begin{array}{c}\text { Hit Count } \\
\text { (\% Hit) }\end{array}$ & $\begin{array}{c}\text { Miss Count } \\
\text { (\% Miss) }\end{array}$ \\
\hline \multirow[t]{12}{*}{$5 \%$} & TCP Bytes & -12 & $51(53 \%)$ & $45(47 \%)$ \\
\hline & TCP Flows & -12 & $54(56 \%)$ & $42(44 \%)$ \\
\hline & TCP Source Address Entropy & -10 & $46(48 \%)$ & $50(52 \%)$ \\
\hline & TCP Destination Address Entropy & -9 & $49(51 \%)$ & $47(49 \%)$ \\
\hline & TCP Source Port Entropy & -10 & $51(53 \%)$ & $45(47 \%)$ \\
\hline & TCP Destination Port Entropy & -14 & $68(71 \%)$ & $28(29 \%)$ \\
\hline & OTHER Bytes & 1 & $72(75 \%)$ & $24(25 \%)$ \\
\hline & OTHER Flows & -11 & $27(28 \%)$ & $69(62 \%)$ \\
\hline & OTHER Source Address Entropy & -13 & $24(25 \%)$ & $72(75 \%)$ \\
\hline & OTHER Destination Address Entropy & -9 & $15(16 \%)$ & $81(84 \%)$ \\
\hline & OTHER Source Port Entropy & -13 & $39(41 \%)$ & $57(43 \%)$ \\
\hline & OTHER Destination Port Entropy & -13 & $21(22 \%)$ & $75(78 \%)$ \\
\hline \multirow{12}{*}{$10 \%$} & TCP Bytes & -12 & $50(52 \%)$ & $46(48 \%)$ \\
\hline & TCP Flows & -12 & $53(55 \%)$ & $43(45 \%)$ \\
\hline & TCP Source Address Entropy & -10 & $45(47 \%)$ & $51(53 \%)$ \\
\hline & TCP Destination Address Entropy & -8 & $51(53 \%)$ & $45(47 \%)$ \\
\hline & TCP Source Port Entropy & -9 & $53(55 \%)$ & $43(45 \%)$ \\
\hline & TCP Destination Port Entropy & -14 & $67(70 \%)$ & $29(30 \%)$ \\
\hline & OTHER Bytes & 1 & $71(74 \%)$ & $25(26 \%)$ \\
\hline & OTHER Flows & -11 & $25(26 \%)$ & $71(74 \%)$ \\
\hline & OTHER Source Address Entropy & -13 & $25(26 \%)$ & $71(74 \%)$ \\
\hline & OTHER Destination Address Entropy & -9 & $16(17 \%)$ & $80(83 \%)$ \\
\hline & OTHER Source Port Entropy & -13 & $38(40 \%)$ & $58(60 \%)$ \\
\hline & OTHER Destination Port Entropy & -12 & $24(25 \%)$ & $72(75 \%)$ \\
\hline \multirow[t]{12}{*}{$20 \%$} & TCP Bytes & -12 & $49(51 \%)$ & $47(49 \%)$ \\
\hline & TCP Flows & -12 & $52(54 \%)$ & $44(46 \%)$ \\
\hline & TCP Source Address Entropy & -9 & $46(48 \%)$ & $50(52 \%)$ \\
\hline & TCP Destination Address Entropy & -8 & $52(54 \%)$ & $44(46 \%)$ \\
\hline & TCP Source Port Entropy & -8 & $55(57 \%)$ & $41(43 \%)$ \\
\hline & TCP Destination Port Entropy & -14 & $66(69 \%)$ & $30(31 \%)$ \\
\hline & OTHER Bytes & 2 & $71(74 \%)$ & $25(26 \%)$ \\
\hline & OTHER Flows & -11 & $23(24 \%)$ & $73(76 \%)$ \\
\hline & OTHER Source Address Entropy & -12 & $24(25 \%)$ & $72(75 \%)$ \\
\hline & OTHER Destination Address Entropy & -8 & $14(15 \%)$ & $82(85 \%)$ \\
\hline & OTHER Source Port Entropy & -12 & $36(38 \%)$ & $60(62 \%)$ \\
\hline & OTHER Destination Port Entropy & -11 & $25(26 \%)$ & $71(74 \%)$ \\
\hline
\end{tabular}

Table 6.17: March 8, 2009 - Wavelet Experiment results 


\subsubsection{Long and Low Infection}

This section examines the results of the Haar Wavelet experiments performed on a low infection rate in a long period of time. The four dates for this particular scenario, outlined in Table 5.2, all had consistent behaviour. As a result, for simplicity, only May 28, 2009 is discussed. Figure 6.14 and Figure 6.15 illustrate the footprints observed with default parameters for the TCP and OTHER features for the $5 \%$ infection ratio (See Table 5.4 for a list of default conditions). The experiments were conducted on test sets of $5 \%, 10 \%$ and $20 \%$ infection ratios. The infected time bins were $81-96$. A number of the feature plots depicted spikes during the infected times. Table 6.18 lists the hit/miss scores and the hit/miss counts measured for each feature. The default threshold method determines a $24 \%$ miss rate in the best feature and about a $54 \%$ miss rate in the worst performing feature. The other threshold methods range from $3 \%$ to $55 \%$, see Section 6.2 .8 for details. 

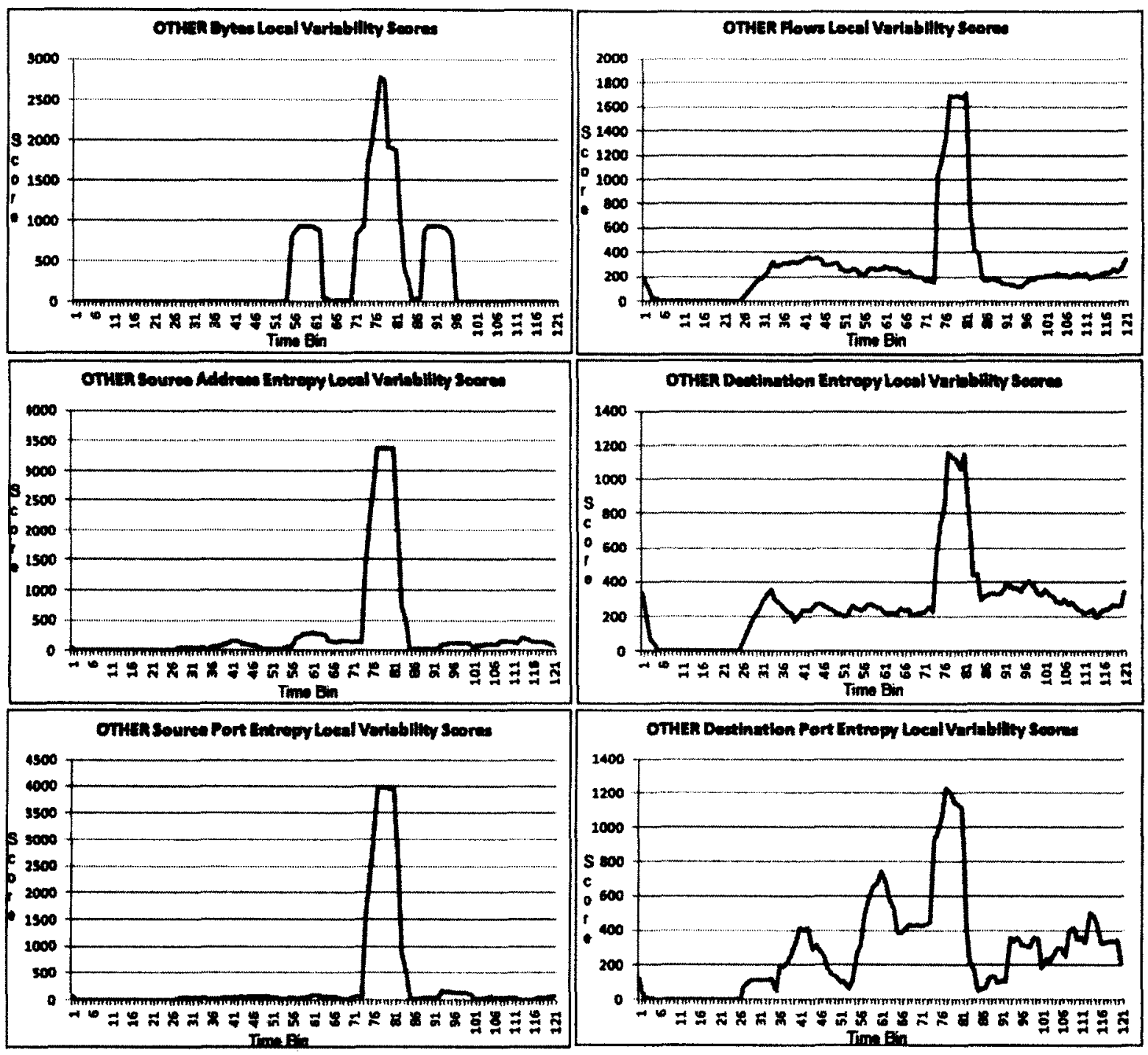

Figure 6.144: May 28, 2009 - Average OTHER Wavelet Local Variability Scores with $5 \%$ infection rate 

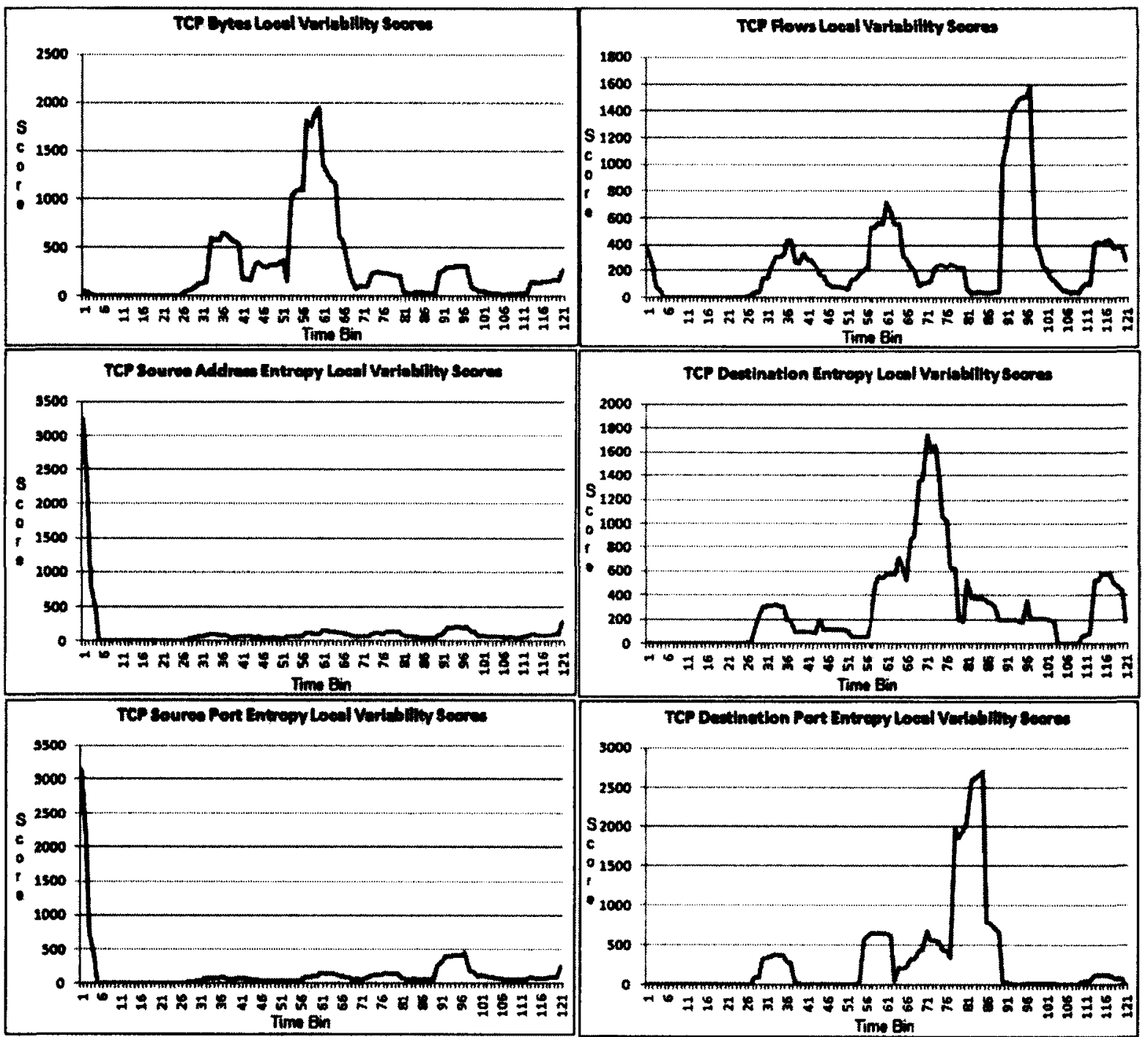

Figure 6.155: May 28, 2009 - Average TCP Wavelet Local Variability Scores with $5 \%$ infection rate 


\begin{tabular}{|c|c|c|c|c|}
\hline $\begin{array}{c}\text { Infection } \\
\text { Rate }\end{array}$ & Feature & Score & $\begin{array}{c}\text { Hit Count } \\
\text { (\% Miss) }\end{array}$ & $\begin{array}{c}\text { Miss Count } \\
\text { (\% Miss) }\end{array}$ \\
\hline \multirow[t]{12}{*}{$5 \%$} & TCP Bytes & -14 & $49(51 \%)$ & $47(49 \%)$ \\
\hline & TCP Flows & -10 & $46(48 \%)$ & $50(52 \%)$ \\
\hline & TCP Source Address Entropy & 0 & $50(52 \%)$ & $46(48 \%)$ \\
\hline & TCP Destination Address Entropy & -14 & $53(55 \%)$ & $43(45 \%)$ \\
\hline & TCP Source Port Entropy & 0 & $49(51 \%)$ & $47(49 \%)$ \\
\hline & TCP Destination Port Entropy & -12 & $64(67 \%)$ & $32(33 \%)$ \\
\hline & OTHER Bytes & -11 & $70(73 \%)$ & $26(27 \%)$ \\
\hline & OTHER Flows & -13 & $39(41 \%)$ & $57(59 \%)$ \\
\hline & OTHER Source Address Entropy & -11 & $71(74 \%)$ & $25(76 \%)$ \\
\hline & OTHER Destination Address Entropy & -11 & $52(54 \%)$ & $44(46 \%)$ \\
\hline & OTHER Source Port Entropy & -11 & $58(60 \%)$ & $38(40 \%)$ \\
\hline & OTHER Destination Port Entropy & -11 & $44(46 \%)$ & $52(54 \%)$ \\
\hline \multirow[t]{12}{*}{$10 \%$} & TCP Bytes & -14 & $48(50 \%)$ & $48(50 \%)$ \\
\hline & TCP Flows & -10 & $45(47 \%)$ & $51(53 \%)$ \\
\hline & TCP Source Address Entropy & 0 & $49(51 \%)$ & $47(49 \%)$ \\
\hline & TCP Destination Address Entropy & -14 & $52(54 \%)$ & $44(46 \%)$ \\
\hline & TCP Source Port Entropy & 0 & $48(50 \%)$ & $48(50 \%)$ \\
\hline & TCP Destination Port Entropy & -11 & $65(68 \%)$ & $31(32 \%)$ \\
\hline & OTHER Bytes & -10 & $70(73 \%)$ & $26(27 \%)$ \\
\hline & OTHER Flows & -12 & $54(56 \%)$ & $42(44 \%)$ \\
\hline & OTHER Source Address Entropy & -10 & $61(64 \%)$ & $35(36 \%)$ \\
\hline & OTHER Destination Address Entropy & -10 & $52(54 \%)$ & $44(46 \%)$ \\
\hline & OTHER Source Port Entropy & -10 & $59(61 \%)$ & $37(39 \%)$ \\
\hline & OTHER Destination Port Entropy & -11 & $42(44 \%)$ & $54(56 \%)$ \\
\hline \multirow[t]{12}{*}{$20 \%$} & TCP Bytes & -14 & $46(48 \%)$ & $50(52 \%)$ \\
\hline & TCP Flows & -10 & $44(46 \%)$ & $52(54 \%)$ \\
\hline & TCP Source Address Entropy & 0 & $47(49 \%)$ & $49(51 \%)$ \\
\hline & TCP Destination Address Entropy & -10 & $54(56 \%)$ & $42(44 \%)$ \\
\hline & TCP Source Port Entropy & 0 & $46(48 \%)$ & $50(52 \%)$ \\
\hline & TCP Destination Port Entropy & -10 & $62(65 \%)$ & $34(35 \%)$ \\
\hline & OTHER Bytes & -11 & $67(70 \%)$ & $29(30 \%)$ \\
\hline & OTHER Flows & -10 & $40(42 \%)$ & $56(58 \%)$ \\
\hline & OTHER Source Address Entropy & -7 & $56(58 \%)$ & $40(42 \%)$ \\
\hline & OTHER Destination Address Entropy & -7 & $59(61 \%)$ & $37(39 \%)$ \\
\hline & OTHER Source Port Entropy & -7 & $54(56 \%)$ & $42(44 \%)$ \\
\hline & OTHER Destination Port Entropy & -7 & $51(53 \%)$ & $45(47 \%)$ \\
\hline
\end{tabular}

Table 6.18: May 28, 2009 - Wavelet Experiment results 


\subsubsection{Short and High Infection}

This section examines the results of the Haar Wavelet experiments performed on a high infection rate in a short period of time. The four dates for this particular scenario, outlined in Table 5.2, all had consistent behaviour. As a result, for simplicity, only January 15, 2009 is discussed. Figure 6.16 and Figure 6.17 illustrate the footprints observed with default parameters for the TCP and OTHER features for the $5 \%$ infection ratio (See Table 5.4 for a list of default conditions). The experiments were conducted on test sets of $75 \%, 100 \%$ and $120 \%$ infection ratios. The infected time bins were during bins $111,112,113$ and 114. A number of the feature plots depicted spikes during the infected times. Table 6.19 lists the hit/miss scores and the hit/miss counts measured for each feature. The default threshold method determines an $18 \%$ miss rate in the best feature and about a $52 \%$ miss rate in the worst performing feature. The other threshold methods range from $4 \%$ to $57 \%$, see Section 6.2 .8 for details. 

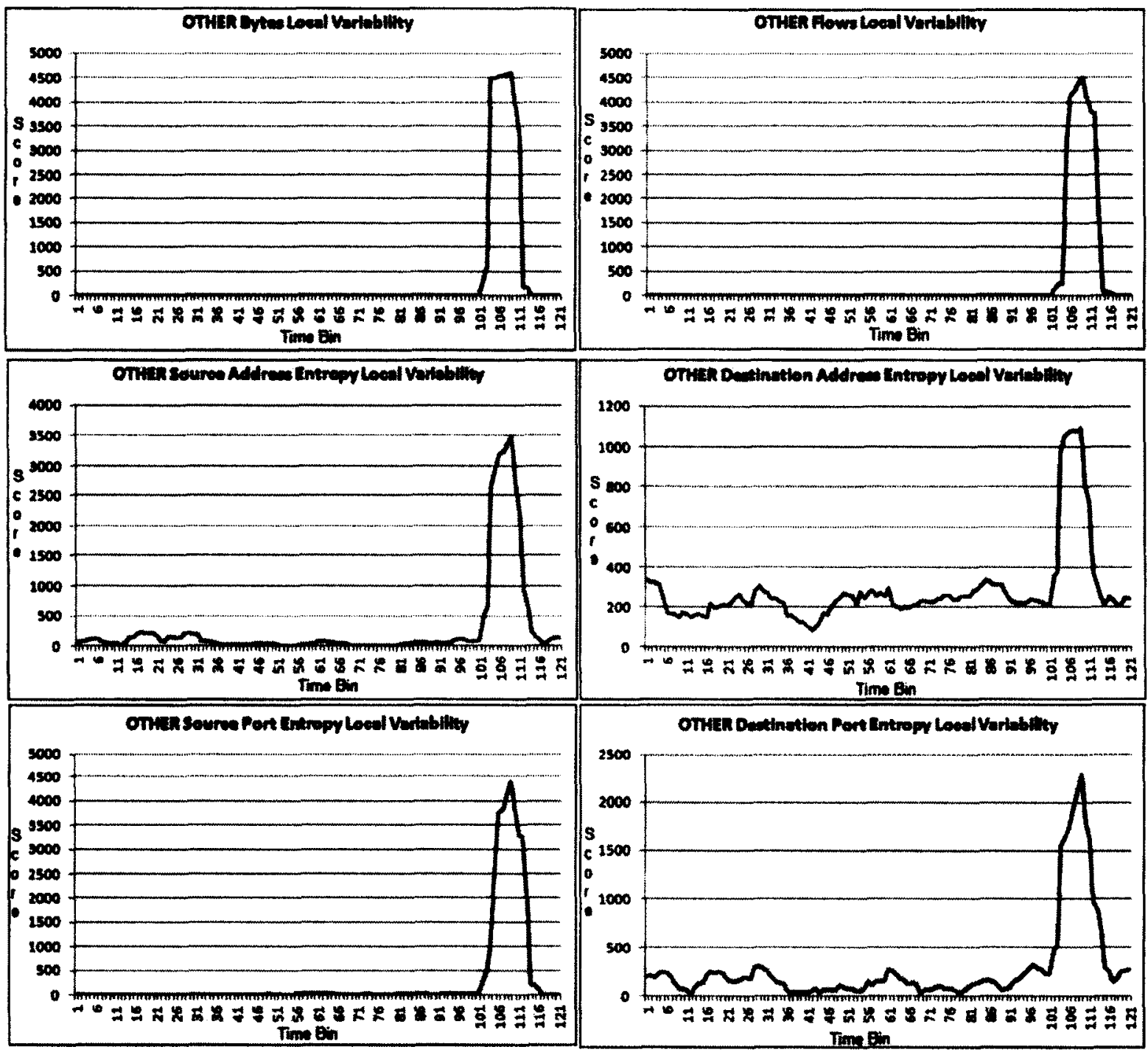

Figure 6.166: January 15, 2009 - Average OTHER Wavelet Local Variability Scores with $5 \%$ infection rate 

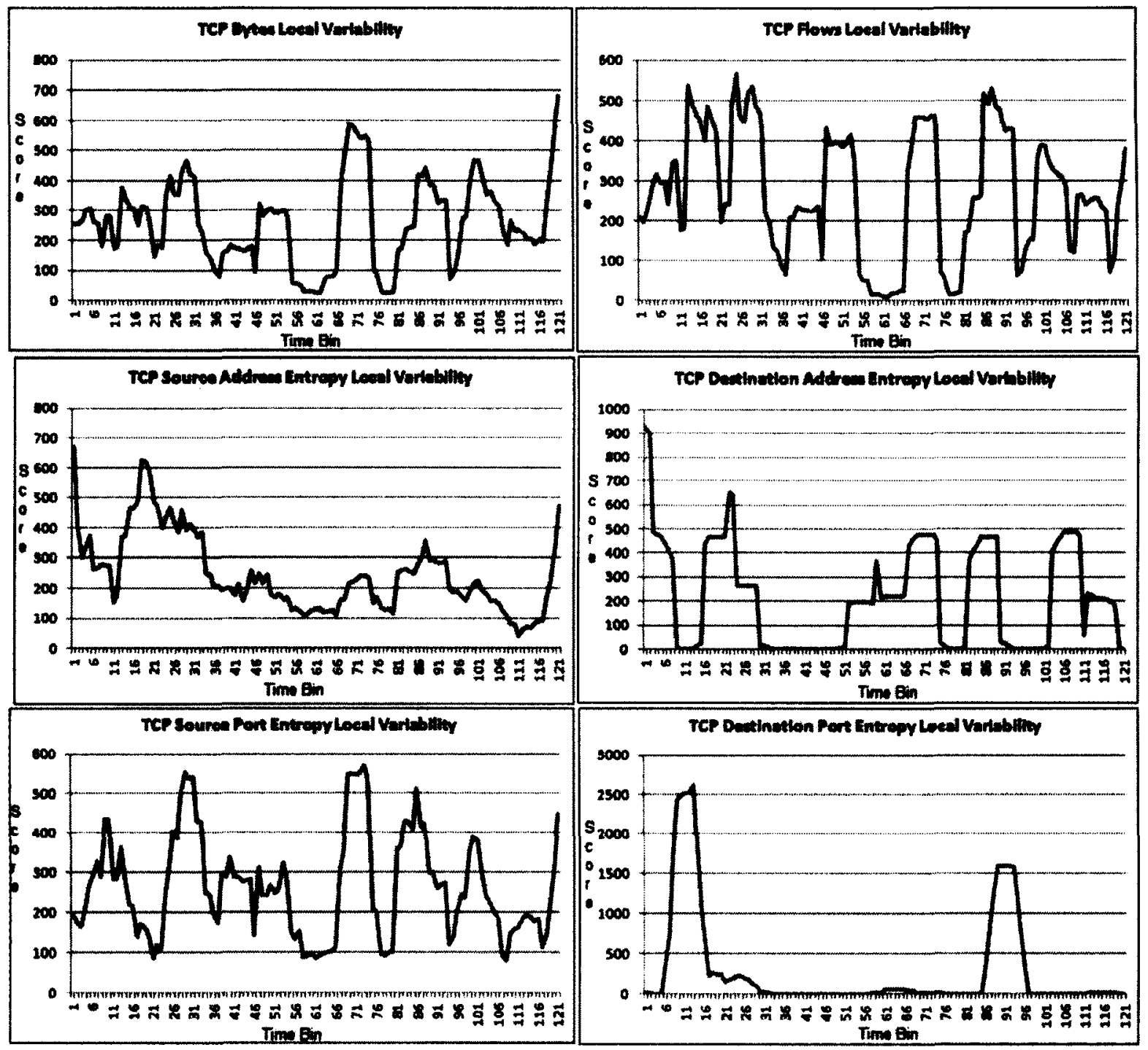

Figure 6.177: January 15, 2009 - Average TCP Wavelet Local Variability Scores with 5\% infection rate 


\begin{tabular}{|c|c|c|c|c|}
\hline $\begin{array}{c}\text { Infection } \\
\text { Rate }\end{array}$ & Feature & Score & $\begin{array}{l}\text { Hit Count } \\
\text { (\% Miss) }\end{array}$ & $\begin{array}{c}\text { Miss Count } \\
\text { (\% Miss) }\end{array}$ \\
\hline \multirow[t]{12}{*}{$75 \%$} & TCP Bytes & 0 & $33(34 \%)$ & $63(66 \%)$ \\
\hline & TCP Flows & 1 & $29(30 \%)$ & $67(70 \%)$ \\
\hline & TCP Source Address Entropy & 0 & $50(52 \%)$ & $46(48 \%)$ \\
\hline & TCP Destination Address Entropy & 0 & $64(67 \%)$ & $32(33 \%)$ \\
\hline & TCP Source Port Entropy & 2 & $43(45 \%)$ & $53(55 \%)$ \\
\hline & TCP Destination Port Entropy & 0 & $70(73 \%)$ & $26(27 \%)$ \\
\hline & OTHER Bytes & 1 & $42(44 \%)$ & $54(56 \%)$ \\
\hline & OTHER Flows & 2 & $35(36 \%)$ & $61(64 \%)$ \\
\hline & OTHER Source Address Entropy & 0 & $37(39 \%)$ & $59(61 \%)$ \\
\hline & OTHER Destination Address Entropy & 0 & $35(36 \%)$ & $61(64 \%)$ \\
\hline & OTHER Source Port Entropy & 2 & $40(42 \%)$ & $56(58 \%)$ \\
\hline & OTHER Destination Port Entropy & 1 & $36(38 \%)$ & $60(62 \%)$ \\
\hline \multirow[t]{12}{*}{$100 \%$} & TCP Bytes & 0 & $32(33 \%)$ & $64(67 \%)$ \\
\hline & TCP Flows & 2 & $30(31 \%)$ & $66(69 \%)$ \\
\hline & TCP Source Address Entropy & 0 & $49(51 \%)$ & $47(49 \%)$ \\
\hline & TCP Destination Address Entropy & 3 & $68(71 \%)$ & $28(29 \%)$ \\
\hline & TCP Source Port Entropy & 3 & $44(46 \%)$ & $52(54 \%)$ \\
\hline & TCP Destination Port Entropy & 0 & $69(72 \%)$ & $27(28 \%)$ \\
\hline & OTHER Bytes & 1 & $45(47 \%)$ & $51(53 \%)$ \\
\hline & OTHER Flows & 2 & $34(35 \%)$ & $62(65 \%)$ \\
\hline & OTHER Source Address Entropy & 1 & $42(44 \%)$ & $54(56 \%)$ \\
\hline & OTHER Destination Address Entropy & 0 & $32(33 \%)$ & $64(67 \%)$ \\
\hline & OTHER Source Port Entropy & 2 & $40(42 \%)$ & $56(58 \%)$ \\
\hline & OTHER Destination Port Entropy & 1 & $35(36 \%)$ & $61(64 \%)$ \\
\hline \multirow[t]{12}{*}{$120 \%$} & TCP Bytes & 0 & $32(33 \%)$ & $64(67 \%)$ \\
\hline & TCP Flows & 2 & $30(31 \%)$ & $66(69 \%)$ \\
\hline & TCP Source Address Entropy & 0 & $49(51 \%)$ & $47(49 \%)$ \\
\hline & TCP Destination Address Entropy & 3 & $74(77 \%)$ & $22(23 \%)$ \\
\hline & TCP Source Port Entropy & 3 & $44(46 \%)$ & $52(54 \%)$ \\
\hline & TCP Destination Port Entropy & 0 & $69(72 \%)$ & $27(28 \%)$ \\
\hline & OTHER Bytes & 1 & $45(47 \%)$ & $51(53 \%)$ \\
\hline & OTHER Flows & 2 & $34(35 \%)$ & $62(65 \%)$ \\
\hline & OTHER Source Address Entropy & 1 & $40(42 \%)$ & $56(58 \%)$ \\
\hline & OTHER Destination Address Entropy & 1 & $33(34 \%)$ & $63(66 \%)$ \\
\hline & OTHER Source Port Entropy & 2 & $39(41 \%)$ & $57(59 \%)$ \\
\hline & OTHER Destination Port Entropy & 4 & $39(41 \%)$ & $57(59 \%)$ \\
\hline
\end{tabular}

Table 6.19: January 15, 2009 - Wavelet Experiment results 


\subsubsection{Long and High Infection}

This section examines the results of the Haar Wavelet experiments performed on a high infection rate in a long period of time. The four dates for this particular scenario, outlined in Table 5.2, all had consistent behaviour. As a result, for simplicity, only June 10, 2009 is discussed. Figure 6.18 and Figure 6.19 illustrate the footprints observed with default parameters for the TCP and OTHER features for the $5 \%$ infection ratio (See Table 5.4 for a list of default conditions). The experiments were conducted on test sets of $75 \%, 100 \%$ and $120 \%$ infection ratios. The infected time bins were during bins $30-40$. A number of the feature plots depicted spikes during the infected times. Table 6.20 lists the hit/miss scores and the hit/miss counts measured for each feature. The default threshold method determines a $12 \%$ miss rate in the best feature and about a $57 \%$ miss rate in the worst performing feature. The other threshold methods range from $4 \%$ to $63 \%$, see Section 6.2 .8 for details. 

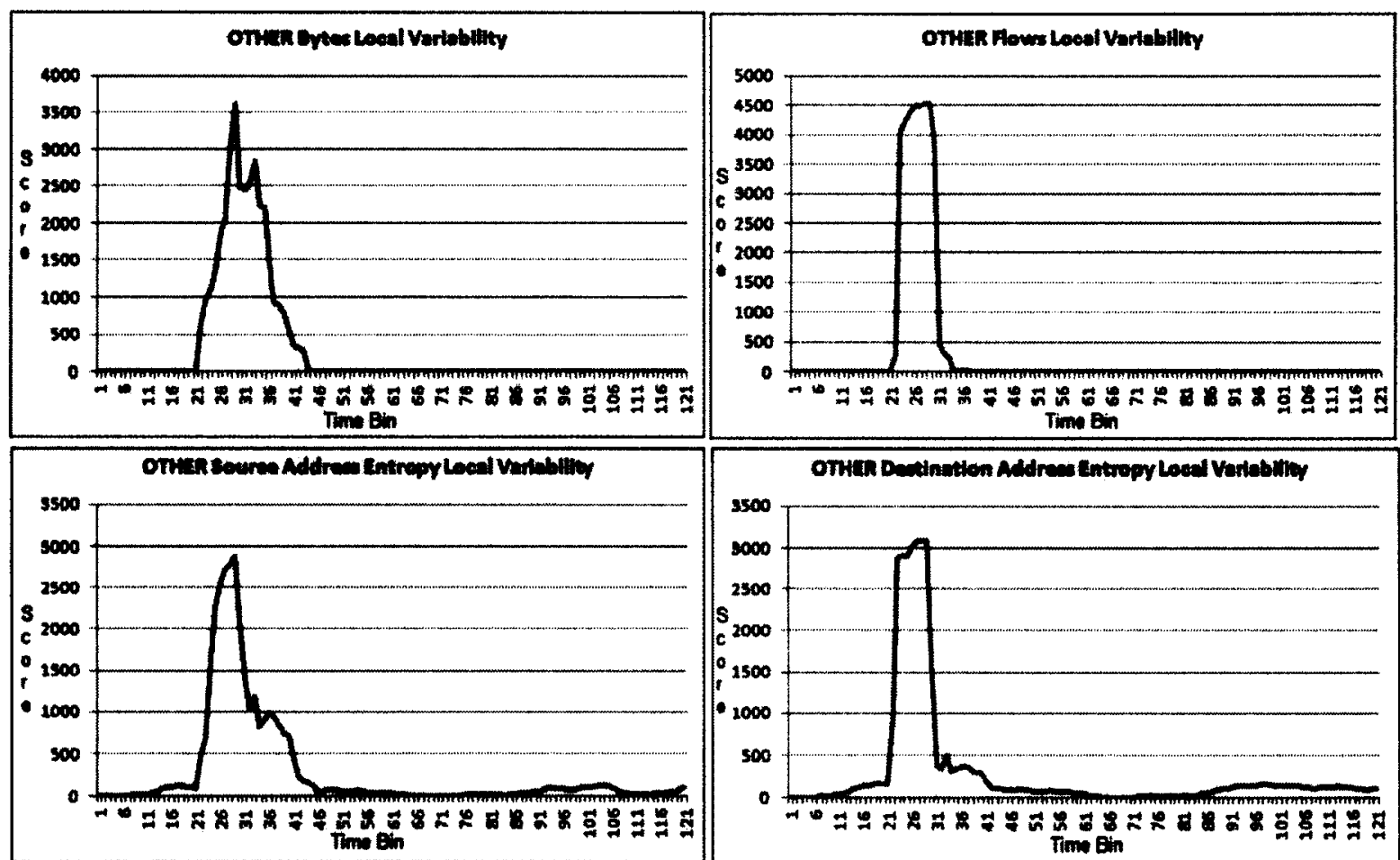

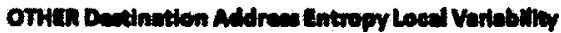
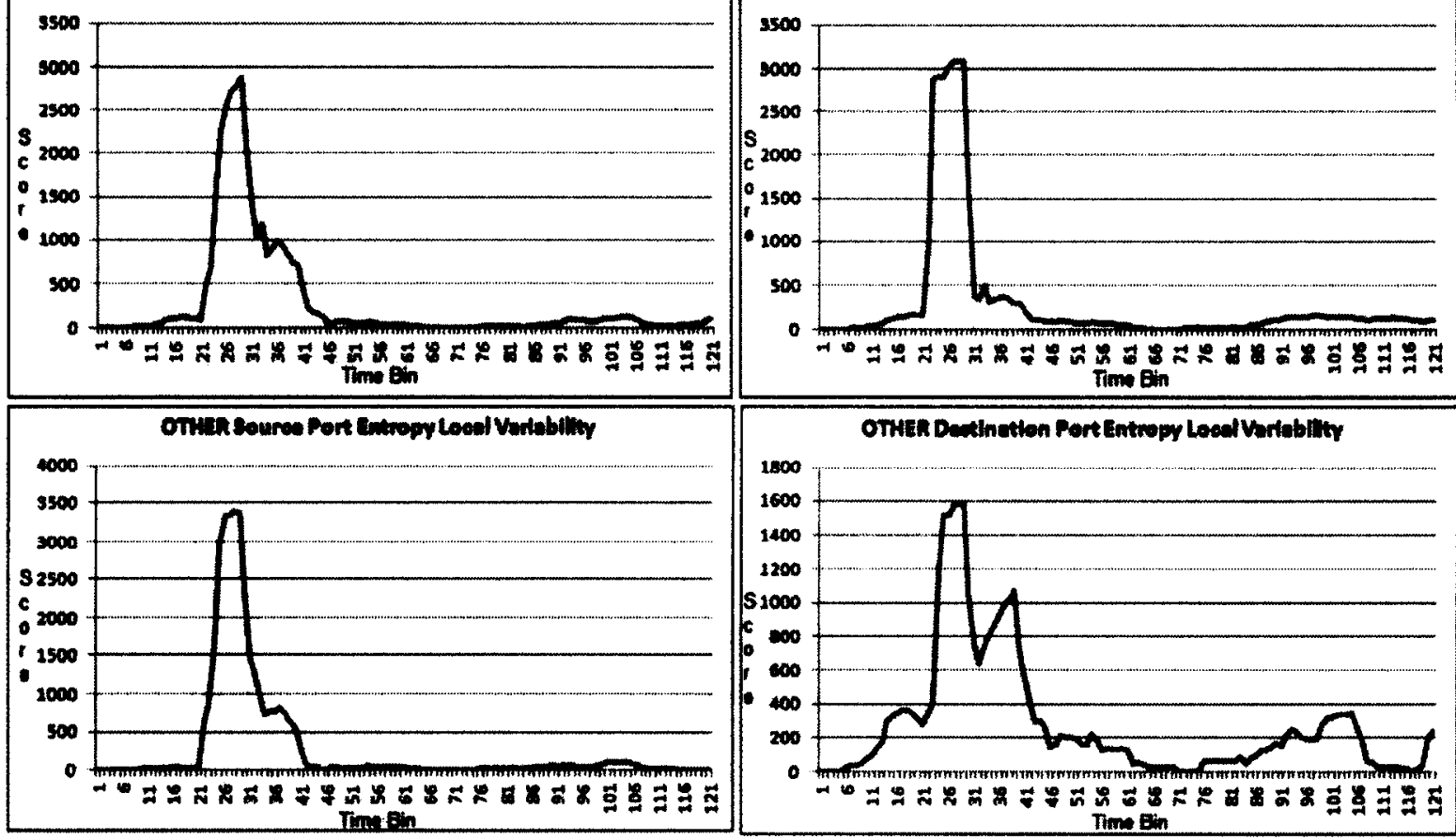

Figure 6.188: June 10, 2009 - Average OTHER Wavelet Local Variability Scores with $5 \%$ infection rate 

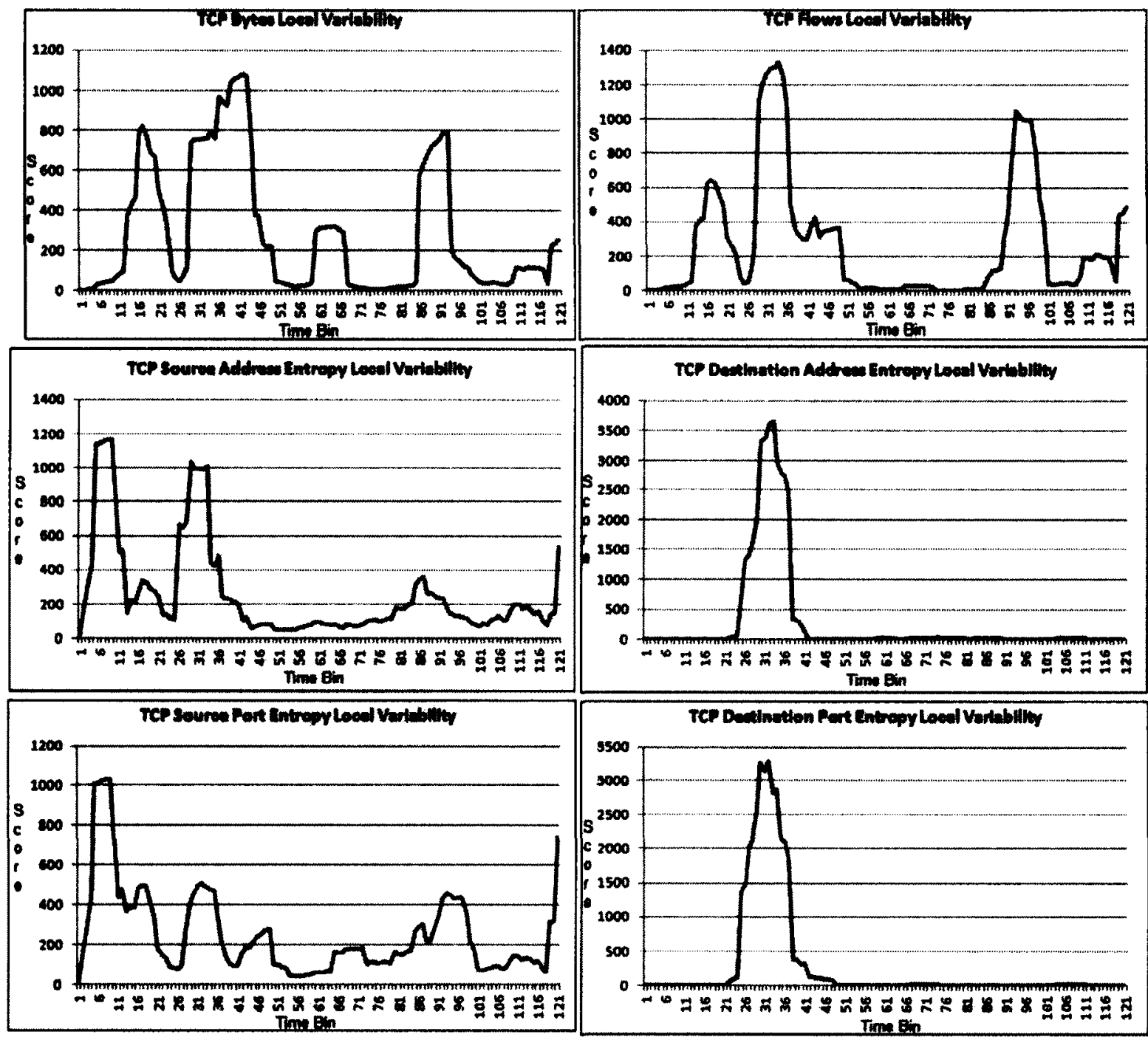

Figure 6.19: June 10, 2009 - Average TCP Wavelet Local Variability Scores with 5\% infection rate 


\begin{tabular}{|c|c|c|c|c|}
\hline $\begin{array}{c}\text { Infection } \\
\text { Rate }\end{array}$ & Feature & Score & $\begin{array}{l}\text { Hit Count } \\
\text { (\% Miss) }\end{array}$ & $\begin{array}{c}\text { Miss Count } \\
\text { (\% Miss) }\end{array}$ \\
\hline \multirow[t]{12}{*}{$75 \%$} & TCP Bytes & -6 & $51(53 \%)$ & $45(47 \%)$ \\
\hline & TCP Flows & -11 & $48(50 \%)$ & $48(50 \%)$ \\
\hline & TCP Source Address Entropy & -14 & $36(38 \%)$ & $60(62 \%)$ \\
\hline & TCP Destination Address Entropy & -3 & $71(74 \%)$ & $25(26 \%)$ \\
\hline & TCP Source Port Entropy & -12 & $35(36 \%)$ & $61(64 \%)$ \\
\hline & TCP Destination Port Entropy & 3 & $72(75 \%)$ & $24(25 \%)$ \\
\hline & OTHER Bytes & 4 & $80(83 \%)$ & $16(17 \%)$ \\
\hline & OTHER Flows & -17 & $57(59 \%)$ & $39(41 \%)$ \\
\hline & OTHER Source Address Entropy & -9 & $27(28 \%)$ & $69(72 \%)$ \\
\hline & OTHER Destination Address Entropy & -18 & $24(25 \%)$ & $72(75 \%)$ \\
\hline & OTHER Source Port Entropy & -9 & $65(68 \%)$ & $31(32 \%)$ \\
\hline & OTHER Destination Port Entropy & -7 & $30(31 \%)$ & $66(69 \%)$ \\
\hline \multirow[t]{12}{*}{$100 \%$} & TCP Bytes & -5 & $52(54 \%)$ & $44(46 \%)$ \\
\hline & TCP Flows & -11 & $49(51 \%)$ & $47(49 \%)$ \\
\hline & TCP Source Address Entropy & -7 & $60(63 \%)$ & $36(27 \%)$ \\
\hline & TCP Destination Address Entropy & -2 & $71(74 \%)$ & $25(26 \%)$ \\
\hline & TCP Source Port Entropy & -12 & $34(35 \%)$ & $62(65 \%)$ \\
\hline & TCP Destination Port Entropy & 6 & $74(77 \%)$ & $22(23 \%)$ \\
\hline & OTHER Bytes & 6 & $81(84 \%)$ & $15(16 \%)$ \\
\hline & OTHER Flows & -17 & $56(58 \%)$ & $40(42 \%)$ \\
\hline & OTHER Source Address Entropy & -9 & $26(27 \%)$ & $70(73 \%)$ \\
\hline & OTHER Destination Address Entropy & -18 & $23(24 \%)$ & $73(76 \%)$ \\
\hline & OTHER Source Port Entropy & -1 & $72(75 \%)$ & $24(25 \%)$ \\
\hline & OTHER Destination Port Entropy & -6 & $34(35 \%)$ & $62(65 \%)$ \\
\hline \multirow[t]{12}{*}{$120 \%$} & TCP Bytes & -4 & $53(55 \%)$ & $43(45 \%)$ \\
\hline & TCP Flows & -12 & $47(49 \%)$ & $49(51 \%)$ \\
\hline & TCP Source Address Entropy & -6 & $51(53 \%)$ & $45(47 \%)$ \\
\hline & TCP Destination Address Entropy & -2 & $70(73 \%)$ & $26(27 \%)$ \\
\hline & TCP Source Port Entropy & -12 & $32(33 \%)$ & $64(67 \%)$ \\
\hline & TCP Destination Port Entropy & 6 & $73(76 \%)$ & $23(24 \%)$ \\
\hline & OTHER Bytes & 6 & $80(83 \%)$ & $16(17 \%)$ \\
\hline & OTHER Flows & -17 & $55(57 \%)$ & $41(43 \%)$ \\
\hline & OTHER Source Address Entropy & -7 & $55(57 \%)$ & $41(43 \%)$ \\
\hline & OTHER Destination Address Entropy & -17 & $24(25 \%)$ & $72(75 \%)$ \\
\hline & OTHER Source Port Entropy & -4 & $68(71 \%)$ & $28(29 \%)$ \\
\hline & OTHER Destination Port Entropy & -3 & $31(32 \%)$ & $65(68 \%)$ \\
\hline
\end{tabular}

Table 6.20: June 10, 2009 - Wavelet Experiment results 


\subsubsection{Varying Dataset size}

A number of experiments varying input dataset size and corresponding filtering boundaries were performed. The results in tables $6.21-6.24$ summarize the evaluation measurements for each scenario on the previously specified dates. It was observed that increasing the dataset size did not improve the hit/miss scores or hit/miss counts.

\begin{tabular}{|c|c|c|c|c|c|}
\hline \multirow[t]{2}{*}{ Size } & \multirow{2}{*}{$\begin{array}{c}\text { Bounds } \\
\text { (High pass, } \\
\text { Mid pass, Low } \\
\text { pass) }\end{array}$} & \multirow[t]{2}{*}{ Feature } & \multicolumn{3}{|c|}{ Miss count } \\
\hline & & & $5 \%$ & $10 \%$ & $20 \%$ \\
\hline \multirow[t]{24}{*}{128} & \multirow[t]{12}{*}{$2,3,2$} & TCP Bytes & 45 & $\overline{46}$ & 47 \\
\hline & & TCP Flows & 42 & 43 & 44 \\
\hline & & TCP Source Address Entropy & 50 & 51 & 50 \\
\hline & & TCP Destination Address Entropy & 47 & 45 & 44 \\
\hline & & TCP Source Port Entropy & 45 & 43 & 41 \\
\hline & & TCP Destination Port Entropy & 28 & 29 & 30 \\
\hline & & OTHER Bytes & 24 & 25 & 25 \\
\hline & & OTHER Flows & 69 & 71 & 73 \\
\hline & & OTHER Source Address Entropy & 72 & 71 & 72 \\
\hline & & OTHER Destination Address Entropy & 81 & 80 & 82 \\
\hline & & OTHER Source Port Entropy & 57 & 58 & 60 \\
\hline & & OTHER Destination Port Entropy & 75 & 72 & 71 \\
\hline & \multirow[t]{12}{*}{$3,2,2$} & TCP Bytes & 43 & 44 & 45 \\
\hline & & TCP Flows & 45 & 46 & 47 \\
\hline & & TCP Source Address Entropy & 59 & 60 & 61 \\
\hline & & TCP Destination Address Entropy & 50 & 49 & 52 \\
\hline & & TCP Source Port Entropy & 52 & 53 & 53 \\
\hline & & TCP Destination Port Entropy & 28 & 29 & 30 \\
\hline & & OTHER Bytes & 24 & 25 & 30 \\
\hline & & OTHER Flows & 72 & 76 & 77 \\
\hline & & OTHER Source Address Entropy & 72 & 73 & 74 \\
\hline & & OTHER Destination Address Entropy & 78 & 79 & 80 \\
\hline & & OTHER Source Port Entropy & 55 & 57 & 60 \\
\hline & & OTHER Destination Port Entropy & 75 & 75 & 75 \\
\hline \multirow[t]{6}{*}{256} & \multirow[t]{6}{*}{$3,2,3$} & TCP Bytes & 65 & 66 & 68 \\
\hline & & TCP Flows & 86 & 87 & 89 \\
\hline & & TCP Source Address Entropy & 111 & 112 & 114 \\
\hline & & TCP Destination Address Entropy & 51 & 50 & 51 \\
\hline & & TCP Source Port Entropy & 100 & 101 & 103 \\
\hline & & TCP Destination Port Entropy & 42 & 43 & 45 \\
\hline
\end{tabular}




\begin{tabular}{|c|c|c|c|c|c|}
\hline & & OTHER Bytes & 25 & 27 & 26 \\
\hline & & OTHER Flows & 138 & 140 & 136 \\
\hline & & OTHER Source Address Entropy & 84 & 85 & 85 \\
\hline & & OTHER Destination Address Entropy & 103 & 106 & 103 \\
\hline & & OTHER Source Port Entropy & 60 & 65 & 65 \\
\hline & & OTHER Destination Port Entropy & 82 & 80 & 80 \\
\hline & $3,3,2$ & TCP Bytes & 65 & 66 & 68 \\
\hline & & TCP Flows & 85 & 86 & 88 \\
\hline & & TCP Source Address Entropy & 109 & 110 & 112 \\
\hline & & TCP Destination Address Entropy & 56 & 50 & 58 \\
\hline & & TCP Source Port Entropy & 97 & 98 & 100 \\
\hline & & TCP Destination Port Entropy & 40 & 41 & 43 \\
\hline & & OTHER Bytes & 26 & 27 & 26 \\
\hline & & OTHER Flows & 138 & 139 & 133 \\
\hline & & OTHER Source Address Entropy & 84 & 85 & 85 \\
\hline & & OTHER Destination Address Entropy & 104 & 105 & 103 \\
\hline & & OTHER Source Port Entropy & 60 & 65 & 65 \\
\hline & & OTHER Destination Port Entropy & 82 & 80 & 80 \\
\hline 512 & $3,3,3$ & TCP Bytes & 109 & 112 & 117 \\
\hline & & TCP Flows & 139 & 142 & 141 \\
\hline & & TCP Source Address Entropy & 233 & 236 & 237 \\
\hline & & TCP Destination Address Entropy & 53 & 45 & 52 \\
\hline & & TCP Source Port Entropy & 223 & 226 & 227 \\
\hline & & TCP Destination Port Entropy & 49 & 53 & 56 \\
\hline & & OTHER Bytes & 29 & 30 & 31 \\
\hline & & OTHER Flows & 238 & 238 & 240 \\
\hline & & OTHER Source Address Entropy & 80 & 84 & 81 \\
\hline & & OTHER Destination Address Entropy & 128 & 135 & 133 \\
\hline & & OTHER Source Port Entropy & 71 & 73 & 70 \\
\hline & & OTHER Destination Port Entropy & 79 & 82 & 82 \\
\hline & $4,3,2$ & TCP Bytes & 107 & 109 & 114 \\
\hline & & TCP Flows & 56 & 158 & 157 \\
\hline & & TCP Source Address Entropy & 246 & 248 & 252 \\
\hline & & TCP Destination Address Entropy & 55 & 51 & 64 \\
\hline & & TCP Source Port Entropy & 238 & 239 & 240 \\
\hline & & TCP Destination Port Entropy & 45 & 47 & 52 \\
\hline & & OTHER Bytes & 30 & 30 & 31 \\
\hline & & OTHER Flows & 239 & 239 & 236 \\
\hline & & OTHER Source Address Entropy & 71 & 72 & 69 \\
\hline & & OTHER Destination Address Entropy & 124 & 132 & 129 \\
\hline & & OTHER Source Port Entropy & 60 & 62 & 66 \\
\hline & & OTHER Destination Port Entropy & 72 & 71 & 73 \\
\hline
\end{tabular}

Table 6.21: March 8, 2009 Varying input data / boundary size results 


\begin{tabular}{|c|c|c|c|c|c|}
\hline \multirow[t]{2}{*}{ Size } & \multirow{2}{*}{$\begin{array}{c}\text { Bounds } \\
\text { (High pass, Mid } \\
\text { pass, Low pass) }\end{array}$} & \multirow[t]{2}{*}{ Feature } & \multicolumn{3}{|c|}{ Miss count } \\
\hline & & & $5 \%$ & $10 \%$ & $20 \%$ \\
\hline \multirow[t]{24}{*}{128} & \multirow[t]{12}{*}{$2,3,2$} & TCP Bytes & 47 & 48 & 50 \\
\hline & & TCP Flows & 50 & 51 & 52 \\
\hline & & TCP Source Address Entropy & 46 & 47 & 49 \\
\hline & & TCP Destination Address Entropy & 43 & 44 & 42 \\
\hline & & TCP Source Port Entropy & 47 & 48 & 50 \\
\hline & & TCP Destination Port Entropy & 32 & 31 & 34 \\
\hline & & OTHER Bytes & 27 & 26 & 29 \\
\hline & & OTHER Flows & 58 & 42 & 56 \\
\hline & & OTHER Source Address Entropy & 36 & 35 & 40 \\
\hline & & OTHER Destination Address Entropy & 45 & 44 & 37 \\
\hline & & OTHER Source Port Entropy & 39 & 37 & 42 \\
\hline & & OTHER Destination Port Entropy & 53 & 54 & 45 \\
\hline & \multirow[t]{12}{*}{$3,2,2$} & TCP Bytes & 47 & 48 & 50 \\
\hline & & TCP Flows & 50 & 51 & 52 \\
\hline & & TCP Source Address Entropy & 44 & 45 & 47 \\
\hline & & TCP Destination Address Entropy & 42 & 43 & 42 \\
\hline & & TCP Source Port Entropy & 39 & 40 & 42 \\
\hline & & TCP Destination Port Entropy & 33 & 32 & 33 \\
\hline & & OTHER Bytes & 30 & 30 & 37 \\
\hline & & OTHER Flows & 63 & 42 & 40 \\
\hline & & OTHER Source Address Entropy & 36 & 35 & 36 \\
\hline & & OTHER Destination Address Entropy & 45 & 44 & 44 \\
\hline & & OTHER Source Port Entropy & 39 & 39 & 40 \\
\hline & & OTHER Destination Port Entropy & 46 & 45 & 46 \\
\hline \multirow[t]{17}{*}{256} & \multirow[t]{12}{*}{$3,2,3$} & TCP Bytes & 89 & 92 & 95 \\
\hline & & TCP Flows & 91 & 94 & 97 \\
\hline & & TCP Source Address Entropy & 76 & 79 & 82 \\
\hline & & TCP Destination Address Entropy & 67 & 70 & 48 \\
\hline & & TCP Source Port Entropy & 73 & 76 & 78 \\
\hline & & TCP Destination Port Entropy & 40 & 37 & 38 \\
\hline & & OTHER Bytes & 49 & 53 & 54 \\
\hline & & OTHER Flows & 93 & 92 & 93 \\
\hline & & OTHER Source Address Entropy & 53 & 55 & 69 \\
\hline & & OTHER Destination Address Entropy & 102 & 103 & 98 \\
\hline & & OTHER Source Port Entropy & 55 & 57 & 66 \\
\hline & & OTHER Destination Port Entropy & 73 & 81 & 71 \\
\hline & \multirow[t]{5}{*}{$3,3,2$} & TCP Bytes & 87 & 90 & 93 \\
\hline & & TCP Flows & 91 & 94 & 98 \\
\hline & & TCP Source Address Entropy & 75 & 78 & 81 \\
\hline & & TCP Destination Address Entropy & 67 & 70 & 48 \\
\hline & & TCP Source Port Entropy & 72 & 75 & 77 \\
\hline
\end{tabular}




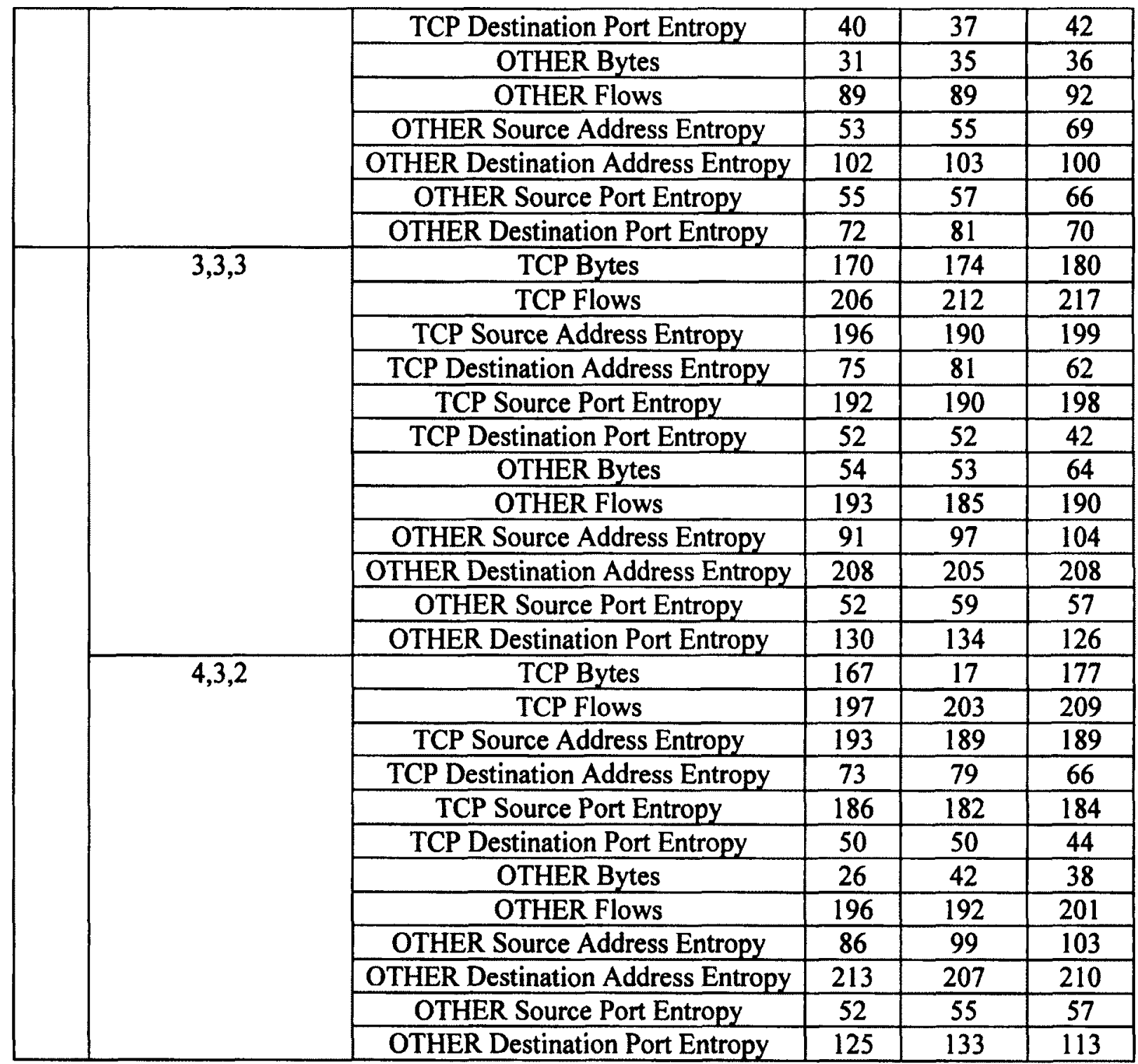

Table 6.22: May 28, 2009 Varying input data / boundary size results 


\begin{tabular}{|c|c|c|c|c|c|}
\hline \multirow[t]{2}{*}{ Size } & \multirow{2}{*}{$\begin{array}{l}\text { Bounds } \\
\text { (High pass, Mid } \\
\text { pass, Low pass) }\end{array}$} & \multirow[t]{2}{*}{ Feature } & \multicolumn{3}{|c|}{ Miss count } \\
\hline & & & $75 \%$ & $100 \%$ & $120 \%$ \\
\hline \multirow[t]{24}{*}{128} & \multirow[t]{12}{*}{$2,3,2$} & TCP Bytes & 63 & 64 & 64 \\
\hline & & TCP Flows & 67 & 66 & 66 \\
\hline & & TCP Source Address Entropy & 46 & 47 & 47 \\
\hline & & TCP Destination Address Entropy & 32 & 28 & 22 \\
\hline & & TCP Source Port Entropy & 53 & 52 & 52 \\
\hline & & TCP Destination Port Entropy & 26 & 27 & 27 \\
\hline & & OTHER Bytes & 54 & 51 & 51 \\
\hline & & OTHER Flows & 61 & 62 & 62 \\
\hline & & OTHER Source Address Entropy & 59 & 51 & 56 \\
\hline & & OTHER Destination Address Entropy & 61 & 64 & 63 \\
\hline & & OTHER Source Port Entropy & 56 & 56 & 57 \\
\hline & & OTHER Destination Port Entropy & 60 & 61 & 57 \\
\hline & \multirow[t]{12}{*}{$3,2,2$} & TCP Bytes & 62 & 63 & 63 \\
\hline & & TCP Flows & 65 & 64 & 64 \\
\hline & & TCP Source Address Entropy & 57 & 58 & 58 \\
\hline & & TCP Destination Address Entropy & 35 & 29 & 22 \\
\hline & & TCP Source Port Entropy & 53 & 52 & 52 \\
\hline & & TCP Destination Port Entropy & 28 & 29 & 30 \\
\hline & & OTHER Bytes & 54 & 55 & 55 \\
\hline & & OTHER Flows & 62 & 63 & 62 \\
\hline & & OTHER Source Address Entropy & 55 & 57 & 55 \\
\hline & & OTHER Destination Address Entropy & 54 & 55 & 54 \\
\hline & & OTHER Source Port Entropy & 61 & 62 & 61 \\
\hline & & OTHER Destination Port Entropy & 61 & 63 & 59 \\
\hline & \multirow[t]{12}{*}{$3,2,3$} & TCP Bytes & 109 & 107 & 108 \\
\hline & & TCP Flows & 104 & 102 & 102 \\
\hline & & TCP Source Address Entropy & 124 & 121 & 121 \\
\hline & & TCP Destination Address Entropy & 42 & 37 & 31 \\
\hline & & TCP Source Port Entropy & 107 & 105 & 105 \\
\hline & & TCP Destination Port Entropy & 47 & 49 & 50 \\
\hline & & OTHER Bytes & 98 & 99 & 100 \\
\hline & & OTHER Flows & 105 & 108 & 109 \\
\hline & & OTHER Source Address Entropy & 69 & 70 & 65 \\
\hline & & OTHER Destination Address Entropy & 124 & 125 & 114 \\
\hline & & OTHER Source Port Entropy & 77 & 79 & 80 \\
\hline & & OTHER Destination Port Entropy & 73 & 77 & 75 \\
\hline & \multirow[t]{5}{*}{$3,3,2$} & TCP Bytes & 110 & 109 & 108 \\
\hline & & TCP Flows & 105 & 104 & 103 \\
\hline & & TCP Source Address Entropy & 123 & 122 & 123 \\
\hline & & TCP Destination Address Entropy & 43 & 36 & 31 \\
\hline & & TCP Source Port Entropy & 106 & 105 & 104 \\
\hline
\end{tabular}




\begin{tabular}{|c|c|c|c|c|}
\hline & TCP Destination Port Entropy & 47 & 48 & 50 \\
\hline & OTHER Bytes & 105 & 105 & 107 \\
\hline & OTHER Flows & 104 & 107 & 109 \\
\hline & OTHER Source Address Entropy & 68 & 69 & 65 \\
\hline & OTHER Destination Address Entropy & 123 & 124 & 114 \\
\hline & OTHER Source Port Entropy & 78 & 78 & 79 \\
\hline & OTHER Destination Port Entropy & 73 & 75 & 75 \\
\hline \multirow[t]{12}{*}{$3,3,3$} & TCP Bytes & 246 & 248 & 249 \\
\hline & TCP Flows & 173 & 173 & 174 \\
\hline & TCP Source Address Entropy & 246 & 244 & 245 \\
\hline & TCP Destination Address Entropy & 50 & 39 & 39 \\
\hline & TCP Source Port Entropy & 241 & 240 & 241 \\
\hline & TCP Destination Port Entropy & 85 & 88 & 86 \\
\hline & OTHER Bytes & 104 & 111 & 108 \\
\hline & OTHER Flows & 221 & 224 & 230 \\
\hline & OTHER Source Address Entropy & 71 & 76 & 74 \\
\hline & OTHER Destination Address Entropy & 217 & 219 & 219 \\
\hline & OTHER Source Port Entropy & 77 & 77 & 87 \\
\hline & OTHER Destination Port Entropy & 82 & 97 & 82 \\
\hline \multirow[t]{12}{*}{$4,3,2$} & TCP Bytes & 236 & 239 & 239 \\
\hline & TCP Flows & 194 & 197 & 197 \\
\hline & TCP Source Address Entropy & 243 & 240 & 240 \\
\hline & TCP Destination Address Entropy & 48 & 41 & 41 \\
\hline & TCP Source Port Entropy & 237 & 238 & 238 \\
\hline & TCP Destination Port Entropy & 76 & 81 & 86 \\
\hline & OTHER Bytes & 118 & 121 & 123 \\
\hline & OTHER Flows & 221 & 228 & 226 \\
\hline & OTHER Source Address Entropy & 73 & 72 & 63 \\
\hline & OTHER Destination Address Entropy & 207 & 213 & 206 \\
\hline & OTHER Source Port Entropy & 84 & 89 & 91 \\
\hline & OTHER Destination Port E & 84 & 98 & 81 \\
\hline
\end{tabular}

Table 6.23: January 15, 2009 Varying input data / boundary size results 


\begin{tabular}{|c|c|c|c|c|c|}
\hline \multirow[t]{2}{*}{ Size } & \multirow{2}{*}{$\begin{array}{l}\text { Bounds } \\
\text { (High pass, Mid } \\
\text { pass, Low pass) }\end{array}$} & \multirow[t]{2}{*}{ Feature } & \multicolumn{3}{|c|}{ Miss count } \\
\hline & & & $75 \%$ & $100 \%$ & $120 \%$ \\
\hline \multirow[t]{24}{*}{128} & \multirow[t]{12}{*}{$2,3,2$} & TCP Bytes & 45 & 44 & $\overline{43}$ \\
\hline & & TCP Flows & 48 & 47 & 49 \\
\hline & & TCP Source Address Entropy & 60 & 36 & 45 \\
\hline & & TCP Destination Address Entropy & 25 & 25 & 26 \\
\hline & & TCP Source Port Entropy & 61 & 62 & 64 \\
\hline & & TCP Destination Port Entropy & 24 & 22 & 23 \\
\hline & & OTHER Bytes & 16 & 15 & 16 \\
\hline & & OTHER Flows & 39 & 40 & 41 \\
\hline & & OTHER Source Address Entropy & 69 & 70 & 41 \\
\hline & & OTHER Destination Address Entropy & 72 & 73 & $\overline{72}$ \\
\hline & & OTHER Source Port Entropy & 31 & 24 & 28 \\
\hline & & OTHER Destination Port Entropy & 66 & 62 & 65 \\
\hline & \multirow[t]{12}{*}{$3,2,2$} & TCP Bytes & 44 & 43 & 42 \\
\hline & & TCP Flows & 42 & 37 & 38 \\
\hline & & TCP Source Address Entropy & 63 & 35 & 47 \\
\hline & & TCP Destination Address Entropy & 32 & 32 & 30 \\
\hline & & TCP Source Port Entropy & 52 & 54 & 55 \\
\hline & & TCP Destination Port Entropy & 27 & 25 & 26 \\
\hline & & OTHER Bytes & 20 & 22 & 20 \\
\hline & & OTHER Flows & 39 & 40 & 41 \\
\hline & & OTHER Source Address Entropy & 75 & 75 & 41 \\
\hline & & OTHER Destination Address Entropy & 75 & 41 & 77 \\
\hline & & OTHER Source Port Entropy & 39 & 32 & 34 \\
\hline & & OTHER Destination Port Entropy & 69 & 70 & 65 \\
\hline & \multirow[t]{12}{*}{$3,2,3$} & TCP Bytes & 95 & 94 & 91 \\
\hline & & TCP Flows & 81 & 78 & 80 \\
\hline & & TCP Source Address Entropy & 121 & 112 & 114 \\
\hline & & TCP Destination Address Entropy & 31 & 28 & 30 \\
\hline & & TCP Source Port Entropy & 114 & 115 & 117 \\
\hline & & TCP Destination Port Entropy & 52 & 33 & 53 \\
\hline & & OTHER Bytes & 35 & 25 & 28 \\
\hline & & OTHER Flows & 57 & 58 & 61 \\
\hline & & OTHER Source Address Entropy & 96 & 95 & 57 \\
\hline & & OTHER Destination Address Entropy & 119 & 119 & 122 \\
\hline & & OTHER Source Port Entropy & 46 & 41 & 46 \\
\hline & & OTHER Destination Port Entropy & 84 & 79 & 80 \\
\hline & \multirow[t]{5}{*}{$3,3,2$} & TCP Bytes & 93 & 92 & 89 \\
\hline & & TCP Flows & 83 & 82 & 80 \\
\hline & & TCP Source Address Entropy & 122 & 113 & 115 \\
\hline & & TCP Destination Address Entropy & 31 & 29 & 31 \\
\hline & & TCP Source Port Entropy & 116 & 117 & 118 \\
\hline
\end{tabular}




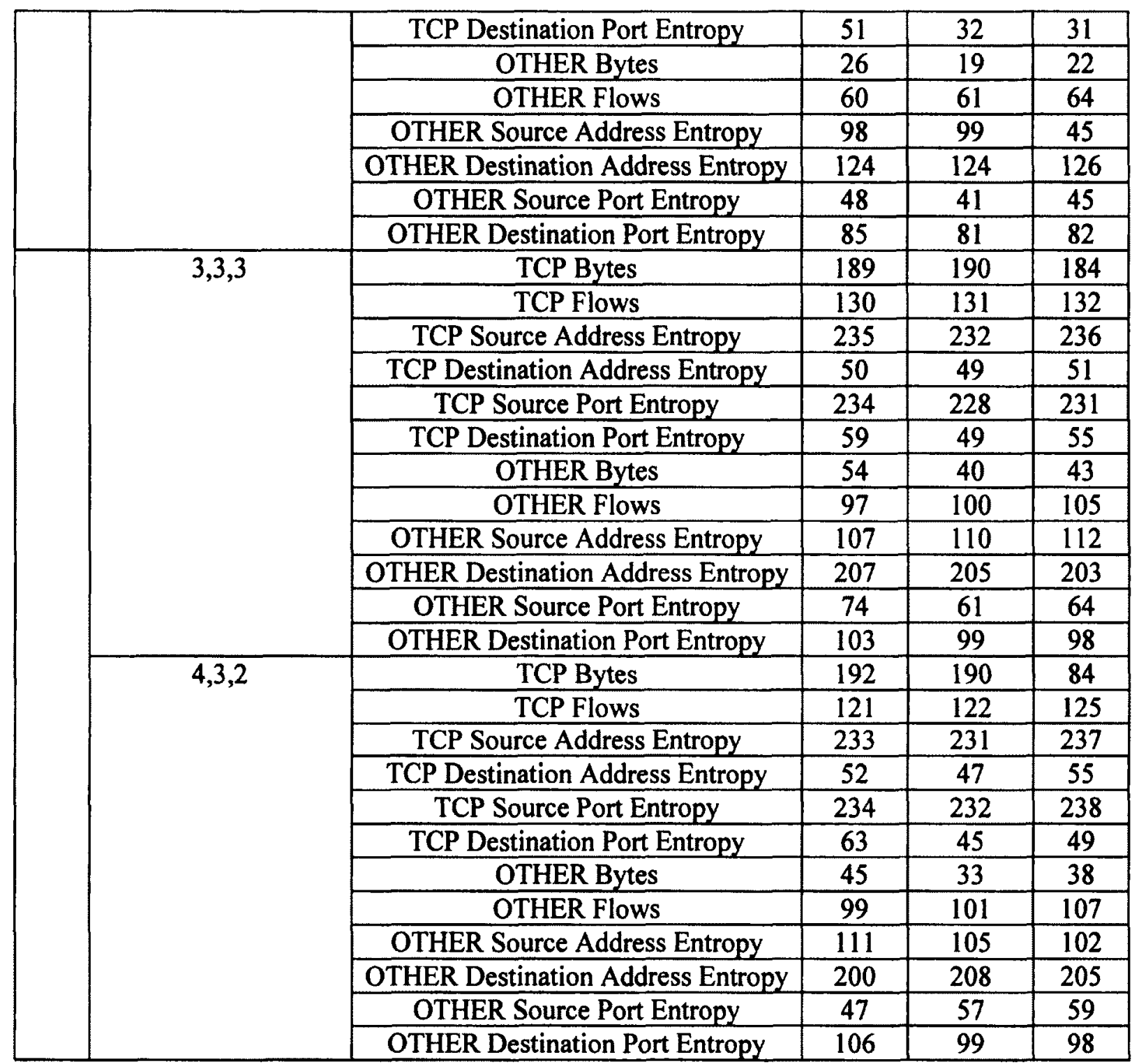

Table 6.24: June 10, 2009 Varying input data / boundary size results

\subsubsection{Varying Local Variability Weights}

A number of experiments were performed using the default parameters, and two different local variability weights. The results in tables $6.25-6.28$ summarize the evaluation measurements for each scenario on the previously specified dates. It was observed that the 
weights, which applied an emphasis to high pass values, had the lowest miss counts best. The miss counts were never worse using weights focusing on the mid pass values.

\begin{tabular}{|c|c|c|c|c|c|}
\hline \multirow{2}{*}{$\begin{array}{l}\text { Bounds } \\
\text { (High pass, } \\
\text { Mid pass, } \\
\text { Low pass) }\end{array}$} & \multirow{2}{*}{$\begin{array}{c}\text { Weights } \\
\text { (High pass, } \\
\text { Mid pass, } \\
\text { Low pass) } \\
\end{array}$} & \multirow[t]{2}{*}{ Features } & \multicolumn{3}{|c|}{ Miss Count } \\
\hline & & & $5 \%$ & $10 \%$ & $20 \%$ \\
\hline \multirow[t]{24}{*}{$2,3,2$} & \multirow[t]{12}{*}{$5,1,0$} & TCP Bytes & 45 & 46 & 47 \\
\hline & & TCP Flows & 42 & 43 & 44 \\
\hline & & TCP Source Address Entropy & 50 & 51 & 50 \\
\hline & & TCP Destination Address Entropy & 47 & 45 & 44 \\
\hline & & TCP Source Port Entropy & 45 & 43 & 41 \\
\hline & & TCP Destination Port Entropy & 28 & 29 & 30 \\
\hline & & OTHER Bytes & 24 & 25 & 25 \\
\hline & & OTHER Flows & 69 & 71 & 73 \\
\hline & & OTHER Source Address Entropy & 72 & 71 & 72 \\
\hline & & OTHER Destination Address Entropy & 81 & 80 & 82 \\
\hline & & OTHER Source Port Entropy & 57 & 58 & 60 \\
\hline & & OTHER Destination Port Entropy & 75 & 72 & 71 \\
\hline & \multirow[t]{12}{*}{$3,2,0$} & TCP Bytes & 44 & 45 & 46 \\
\hline & & TCP Flows & 41 & 42 & 43 \\
\hline & & TCP Source Address Entropy & 51 & 52 & 53 \\
\hline & & TCP Destination Address Entropy & 46 & 45 & 46 \\
\hline & & TCP Source Port Entropy & 51 & 52 & 53 \\
\hline & & TCP Destination Port Entropy & 28 & 29 & 30 \\
\hline & & OTHER Bytes & 25 & 31 & 32 \\
\hline & & OTHER Flows & 68 & 67 & 70 \\
\hline & & OTHER Source Address Entropy & 71 & 72 & 73 \\
\hline & & OTHER Destination Address Entropy & 77 & 80 & 80 \\
\hline & & OTHER Source Port Entropy & 53 & 57 & 59 \\
\hline & & OTHER Destination Port Entropy & 75 & 74 & 74 \\
\hline \multirow[t]{12}{*}{$3,2,2$} & \multirow[t]{12}{*}{$5,1,0$} & TCP Bytes & 43 & 44 & 45 \\
\hline & & TCP Flows & 45 & 46 & 47 \\
\hline & & TCP Source Address Entropy & 59 & 60 & 61 \\
\hline & & TCP Destination Address Entropy & 50 & 49 & 52 \\
\hline & & TCP Source Port Entropy & 52 & 53 & 53 \\
\hline & & TCP Destination Port Entropy & 28 & 29 & 30 \\
\hline & & OTHER Bytes & 24 & 25 & 30 \\
\hline & & OTHER Flows & 72 & 76 & 77 \\
\hline & & OTHER Source Address Entropy & 72 & 73 & 74 \\
\hline & & OTHER Destination Address Entropy & 78 & 79 & 80 \\
\hline & & OTHER Source Port Entropy & 55 & 57 & 60 \\
\hline & & OTHER Destination Port Entropy & 75 & 75 & 75 \\
\hline
\end{tabular}




\begin{tabular}{|c|c|c|c|c|}
\hline \multirow{12}{*}{ 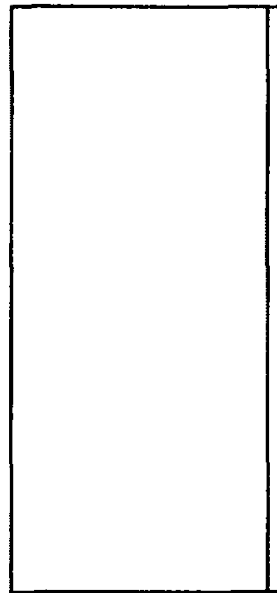 } & TCP Bytes & 43 & 44 & 45 \\
\hline & TCP Flows & 42 & 43 & 44 \\
\hline & TCP Source Address Entropy & 47 & 48 & 49 \\
\hline & TCP Destination Address Entropy & 52 & 50 & 50 \\
\hline & TCP Source Port Entropy & 49 & 50 & 50 \\
\hline & TCP Destination Port Entropy & 28 & 29 & 30 \\
\hline & OTHER Bytes & 30 & 28 & 24 \\
\hline & OTHER Flows & 64 & 66 & 67 \\
\hline & OTHER Source Address Entropy & 72 & 73 & 70 \\
\hline & OTHER Destination Address Entropy & 82 & 82 & 78 \\
\hline & OTHER Source Port Entropy & 54 & 54 & 57 \\
\hline & OTHER Destination Port Entropy & 75 & 74 & 74 \\
\hline
\end{tabular}

Table 6.25: March 8, 2009 Varying Local Variability weights results 


\begin{tabular}{|c|c|c|c|c|c|}
\hline \multirow{2}{*}{$\begin{array}{c}\text { Bounds } \\
\text { (High pass, } \\
\text { Mid pass, } \\
\text { Low pass) }\end{array}$} & \multirow{2}{*}{$\begin{array}{c}\text { Weights } \\
\text { (High pass, } \\
\text { Mid pass, } \\
\text { Low pass) }\end{array}$} & \multirow[t]{2}{*}{ Features } & \multicolumn{3}{|c|}{ Miss Count } \\
\hline & & & $5 \%$ & $10 \%$ & $20 \%$ \\
\hline \multirow[t]{24}{*}{$2,3,2$} & \multirow[t]{12}{*}{$5,1,0$} & TCP Bytes & 47 & 48 & 50 \\
\hline & & TCP Flows & 50 & 51 & 52 \\
\hline & & TCP Source Address Entropy & 46 & 47 & 49 \\
\hline & & TCP Destination Address Entropy & 43 & 44 & 42 \\
\hline & & TCP Source Port Entropy & 47 & 48 & 50 \\
\hline & & TCP Destination Port Entropy & 32 & 31 & 34 \\
\hline & & OTHER Bytes & 27 & 26 & 29 \\
\hline & & OTHER Flows & 58 & 42 & 56 \\
\hline & & OTHER Source Address Entropy & 36 & 35 & 40 \\
\hline & & OTHER Destination Address Entropy & 45 & 44 & 37 \\
\hline & & OTHER Source Port Entropy & 39 & 37 & 42 \\
\hline & & OTHER Destination Port Entropy & 53 & 54 & 45 \\
\hline & \multirow[t]{12}{*}{$3,2,0$} & TCP Bytes & 48 & 49 & 51 \\
\hline & & TCP Flows & 49 & 50 & 52 \\
\hline & & TCP Source Address Entropy & 47 & 48 & 50 \\
\hline & & TCP Destination Address Entropy & 42 & 43 & 37 \\
\hline & & TCP Source Port Entropy & 47 & 48 & 50 \\
\hline & & TCP Destination Port Entropy & 33 & 32 & 29 \\
\hline & & OTHER Bytes & 27 & 26 & 28 \\
\hline & & OTHER Flows & 62 & 36 & 60 \\
\hline & & OTHER Source Address Entropy & 36 & 36 & 43 \\
\hline & & OTHER Destination Address Entropy & 49 & 47 & 45 \\
\hline & & OTHER Source Port Entropy & 39 & 39 & 41 \\
\hline & & OTHER Destination Port Entropy & 46 & 45 & 43 \\
\hline \multirow[t]{16}{*}{$3,2,2$} & \multirow[t]{12}{*}{$5,1,0$} & TCP Bytes & 47 & 48 & 50 \\
\hline & & TCP Flows & 50 & 51 & 52 \\
\hline & & TCP Source Address Entropy & 44 & 45 & 47 \\
\hline & & TCP Destination Address Entropy & 42 & 43 & 42 \\
\hline & & TCP Source Port Entropy & 39 & 40 & 42 \\
\hline & & TCP Destination Port Entropy & 33 & 32 & 33 \\
\hline & & OTHER Bytes & 30 & 30 & 37 \\
\hline & & OTHER Flows & 63 & 42 & 40 \\
\hline & & OTHER Source Address Entropy & 36 & 35 & 36 \\
\hline & & OTHER Destination Address Entropy & 45 & 44 & 44 \\
\hline & & OTHER Source Port Entropy & 39 & 39 & 40 \\
\hline & & OTHER Destination Port Entropy & 46 & 45 & 46 \\
\hline & \multirow[t]{4}{*}{$3,2,0$} & TCP Bytes & 46 & 45 & 48 \\
\hline & & TCP Flows & 50 & 49 & 52 \\
\hline & & TCP Source Address Entropy & 46 & 45 & 48 \\
\hline & & TCP Destination Address Entropy & 44 & 43 & 37 \\
\hline
\end{tabular}




\begin{tabular}{|c|c|c|c|c|c|}
\hline & \multirow{8}{*}{ 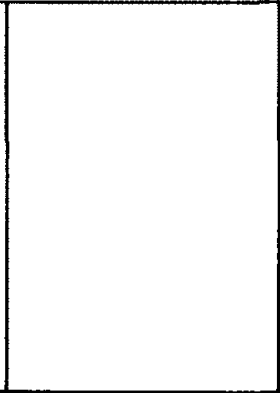 } & TCP Source Port Entropy & 46 & 45 & 48 \\
\hline & & TCP Destination Port Entropy & 33 & 34 & 30 \\
\hline & & OTHER Bytes & 31 & 28 & 31 \\
\hline & & OTHER Flows & 36 & 62 & 38 \\
\hline & & OTHER Source Address Entropy & 36 & 36 & 37 \\
\hline & & OTHER Destination Address Entropy & 46 & 46 & 45 \\
\hline & & OTHER Source Port Entropy & 37 & 39 & 38 \\
\hline & & OTHER Destination Port Entropy & 48 & 56 & 45 \\
\hline
\end{tabular}

Table 6.26: May 28, 2009 Varying Local Variability weights results 


\begin{tabular}{|c|c|c|c|c|c|}
\hline \multirow{2}{*}{\begin{tabular}{|c|} 
Bounds \\
(High pass, \\
Mid pass, Low \\
pass) \\
\end{tabular}} & \multirow{2}{*}{$\begin{array}{l}\text { Weights } \\
\text { (High pass, } \\
\text { Mid pass, } \\
\text { Low pass) }\end{array}$} & \multirow[t]{2}{*}{ Features } & \multicolumn{3}{|c|}{ Miss Count } \\
\hline & & & $\begin{array}{l}75 \\
\%\end{array}$ & $100 \%$ & $120 \%$ \\
\hline \multirow[t]{24}{*}{$2,3,2$} & \multirow[t]{12}{*}{$5,1,0$} & TCP Bytes & 63 & 64 & 64 \\
\hline & & TCP Flows & 67 & 66 & 66 \\
\hline & & TCP Source Address Entropy & 46 & 47 & 47 \\
\hline & & TCP Destination Address Entropy & 32 & 28 & 22 \\
\hline & & TCP Source Port Entropy & 53 & 52 & 52 \\
\hline & & TCP Destination Port Entropy & 26 & 27 & 27 \\
\hline & & OTHER Bytes & 54 & 51 & 51 \\
\hline & & OTHER Flows & 61 & 62 & 62 \\
\hline & & OTHER Source Address Entropy & 59 & 51 & 56 \\
\hline & & OTHER Destination Address Entropy & 61 & 64 & 63 \\
\hline & & OTHER Source Port Entropy & 56 & 56 & 57 \\
\hline & & OTHER Destination Port Entropy & 60 & 61 & 57 \\
\hline & \multirow[t]{12}{*}{$3,2,0$} & TCP Bytes & 58 & 59 & 59 \\
\hline & & TCP Flows & 64 & 63 & 63 \\
\hline & & TCP Source Address Entropy & 50 & 51 & 51 \\
\hline & & TCP Destination Address Entropy & 29 & 29 & 22 \\
\hline & & TCP Source Port Entropy & 58 & 57 & 57 \\
\hline & & TCP Destination Port Entropy & 25 & 26 & 26 \\
\hline & & OTHER Bytes & 49 & 49 & 49 \\
\hline & & OTHER Flows & 58 & 59 & 56 \\
\hline & & OTHER Source Address Entropy & 53 & 54 & 55 \\
\hline & & OTHER Destination Address Entropy & 55 & 56 & 55 \\
\hline & & OTHER Source Port Entropy & 54 & 56 & 57 \\
\hline & & OTHER Destination Port Entropy & 63 & 63 & 57 \\
\hline \multirow[t]{16}{*}{$3,2,2$} & \multirow[t]{12}{*}{$5,1,0$} & TCP Bytes & 62 & 63 & 63 \\
\hline & & TCP Flows & 65 & 64 & 64 \\
\hline & & TCP Source Address Entropy & 57 & 58 & 58 \\
\hline & & TCP Destination Address Entropy & 35 & 29 & 22 \\
\hline & & TCP Source Port Entropy & 53 & 52 & 52 \\
\hline & & TCP Destination Port Entropy & 28 & 29 & 30 \\
\hline & & OTHER Bytes & 54 & 55 & 55 \\
\hline & & OTHER Flows & 62 & 63 & 62 \\
\hline & & OTHER Source Address Entropy & 55 & 57 & 55 \\
\hline & & OTHER Destination Address Entropy & 54 & 55 & 54 \\
\hline & & OTHER Source Port Entropy & 61 & 62 & 61 \\
\hline & & OTHER Destination Port Entropy & 61 & 63 & 59 \\
\hline & \multirow[t]{4}{*}{$3,2,0$} & TCP Bytes & 60 & 61 & 61 \\
\hline & & TCP Flows & 64 & 63 & 63 \\
\hline & & TCP Source Address Entropy & 55 & 56 & 56 \\
\hline & & TCP Destination Address Entropy & 28 & 31 & 25 \\
\hline
\end{tabular}




\begin{tabular}{|c|c|c|c|c|c|}
\hline & \multirow{8}{*}{ 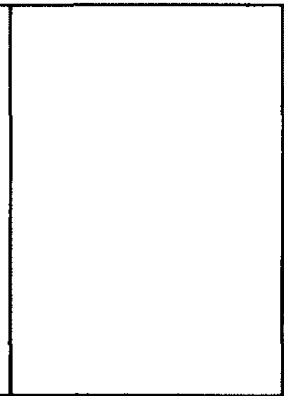 } & TCP Source Port Entropy & 61 & 60 & 60 \\
\hline & & TCP Destination Port Entropy & 28 & 29 & 29 \\
\hline & & OTHER Bytes & 59 & 60 & 60 \\
\hline & & OTHER Flows & 60 & 60 & 56 \\
\hline & & OTHER Source Address Entropy & 60 & 56 & 55 \\
\hline & & OTHER Destination Address Entropy & 57 & 60 & 59 \\
\hline & & OTHER Source Port Entropy & 62 & 61 & 58 \\
\hline & & OTHER Destination Port Entropy & 66 & 65 & 61 \\
\hline
\end{tabular}

Table 6.27: January 15, 2009 Varying Local Variability weights results 


\begin{tabular}{|c|c|c|c|c|c|}
\hline \multirow{2}{*}{$\begin{array}{c}\text { Bounds } \\
\text { (High pass, } \\
\text { Mid pass, Low } \\
\text { pass) }\end{array}$} & \multirow{2}{*}{$\begin{array}{l}\text { Weights } \\
\text { (High pass, } \\
\text { Mid pass, } \\
\text { Low pass) } \\
\end{array}$} & \multirow[t]{2}{*}{ Features } & \multicolumn{3}{|c|}{ Miss Count } \\
\hline & & & $75 \%$ & $100 \%$ & $120 \%$ \\
\hline \multirow[t]{24}{*}{$2,3,2$} & \multirow[t]{12}{*}{$5,1,0$} & TCP Bytes & 45 & 44 & 43 \\
\hline & & TCP Flows & 48 & 47 & 49 \\
\hline & & TCP Source Address Entropy & 60 & 36 & 45 \\
\hline & & TCP Destination Address Entropy & $\frac{25}{25}$ & 25 & 26 \\
\hline & & TCP Source Port Entropy & 61 & 62 & 64 \\
\hline & & TCP Destination Port Entropy & 24 & 22 & 23 \\
\hline & & OTHER Bytes & 16 & 15 & 16 \\
\hline & & OTHER Flows & 39 & 40 & 41 \\
\hline & & OTHER Source Address Entropy & 69 & 70 & 41 \\
\hline & & OTHER Destination Address Entropy & 72 & 73 & 72 \\
\hline & & OTHER Source Port Entropy & 31 & 24 & 28 \\
\hline & & OTHER Destination Port Entropy & 66 & 62 & 65 \\
\hline & \multirow[t]{12}{*}{$3,2,0$} & TCP Bytes & 46 & 45 & 44 \\
\hline & & TCP Flows & 49 & 45 & 47 \\
\hline & & TCP Source Address Entropy & 61 & 47 & 57 \\
\hline & & TCP Destination Address Entropy & 24 & 24 & 22 \\
\hline & & TCP Source Port Entropy & 64 & 65 & 66 \\
\hline & & TCP Destination Port Entropy & 24 & 22 & 23 \\
\hline & & OTHER Bytes & 16 & 15 & 15 \\
\hline & & OTHER Flows & 39 & 40 & 41 \\
\hline & & OTHER Source Address Entropy & 70 & 74 & 38 \\
\hline & & OTHER Destination Address Entropy & 66 & 75 & 70 \\
\hline & & OTHER Source Port Entropy & 31 & 25 & 28 \\
\hline & & OTHER Destination Port Entropy & 63 & 63 & 63 \\
\hline \multirow[t]{16}{*}{$3,2,2$} & \multirow[t]{12}{*}{$5,1,0$} & TCP Bytes & 44 & 43 & 42 \\
\hline & & TCP Flows & 42 & 37 & 38 \\
\hline & & TCP Source Address Entropy & 63 & 35 & 47 \\
\hline & & TCP Destination Address Entropy & 32 & 32 & 30 \\
\hline & & TCP Source Port Entropy & 52 & 54 & 55 \\
\hline & & TCP Destination Port Entropy & 27 & 25 & 26 \\
\hline & & OTHER Bytes & 20 & 22 & 20 \\
\hline & & OTHER Flows & 39 & 40 & 41 \\
\hline & & OTHER Source Address Entropy & 75 & 75 & 41 \\
\hline & & OTHER Destination Address Entropy & 75 & 41 & 77 \\
\hline & & OTHER Source Port Entropy & 39 & 32 & 34 \\
\hline & & OTHER Destination Port Entropy & 69 & 70 & 65 \\
\hline & \multirow[t]{4}{*}{$3,2,0$} & TCP Bytes & 46 & 45 & 44 \\
\hline & & TCP Flows & 44 & 42 & 43 \\
\hline & & TCP Source Address Entropy & 62 & 56 & 58 \\
\hline & & TCP Destination Address Entropy & 28 & 31 & 28 \\
\hline
\end{tabular}




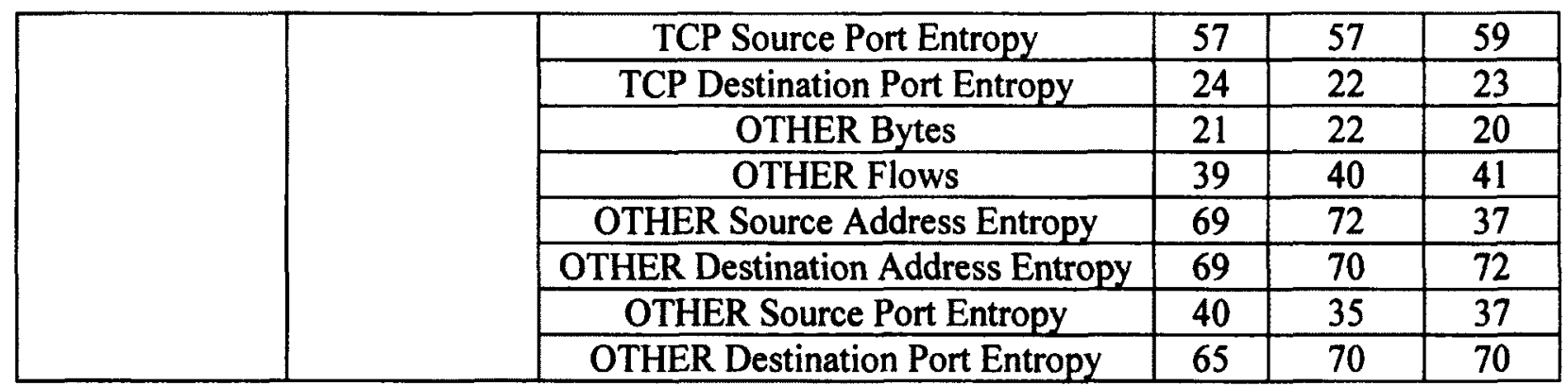

Table 6.28: June 10, 2009 - Varying Local Variability weights results 


\subsubsection{Threshold Techniques and Haar Wavelet Filtering analysis}

Each of the experiments performed used four different threshold techniques to automatically identify anomalous time bins. The results listed in tables $6.29-6.32$ summarize the evaluation measurements for threshold techniques on the specified dates. In each scenario, at least one feature was able to detect the infected time. It was observed that all the dynamic averages performed worse than the static average.

\begin{tabular}{|c|c|c|c|c|c|}
\hline \multirow{2}{*}{$\begin{array}{c}\text { Infection } \\
\text { Rate }\end{array}$} & \multirow[t]{2}{*}{ Feature } & \multicolumn{4}{|c|}{ Miss Count } \\
\hline & & $\begin{array}{c}\text { Static } \\
\text { Average }\end{array}$ & $\begin{array}{l}\text { Moving } \\
\text { Average } \\
\text { without } \\
\text { Outliers }\end{array}$ & $\begin{array}{l}\text { Moving } \\
\text { Average }\end{array}$ & $\begin{array}{c}\text { Weighted } \\
\text { Moving } \\
\text { Average }\end{array}$ \\
\hline \multirow[t]{12}{*}{$5 \%$} & TCP Bytes & 33 & 45 & 61 & 49 \\
\hline & TCP Flows & 29 & 42 & 56 & 38 \\
\hline & TCP Source Address Entropy & 26 & 50 & 58 & 44 \\
\hline & TCP Destination Address Entropy & 30 & 47 & 71 & 50 \\
\hline & TCP Source Port Entropy & 32 & 45 & 53 & 39 \\
\hline & TCP Destination Port Entropy & 10 & 28 & 65 & 38 \\
\hline & OTHER Bytes & 7 & 24 & 50 & 38 \\
\hline & OTHER Flows & 8 & 69 & 70 & 70 \\
\hline & OTHER Source Address Entropy & 16 & 72 & 79 & 74 \\
\hline & OTHER Destination Address Entropy & 18 & 81 & 79 & 81 \\
\hline & OTHER Source Port Entropy & 10 & 57 & 76 & 68 \\
\hline & OTHER Destination Port Entropy & 57 & 75 & 79 & 76 \\
\hline \multirow[t]{12}{*}{$10 \%$} & TCP Bytes & 34 & 46 & 62 & 50 \\
\hline & TCP Flows & 30 & 43 & 57 & 39 \\
\hline & TCP Source Address Entropy & 27 & 51 & 59 & 45 \\
\hline & TCP Destination Address Entropy & 17 & $\overline{45}$ & 70 & 50 \\
\hline & TCP Source Port Entropy & 33 & 43 & 52 & 38 \\
\hline & TCP Destination Port Entropy & 11 & 29 & 64 & 41 \\
\hline & OTHER Bytes & 8 & 25 & 52 & 40 \\
\hline & OTHER Flows & 8 & 71 & 73 & 70 \\
\hline & OTHER Source Address Entropy & 12 & 71 & 79 & 74 \\
\hline & OTHER Destination Address Entropy & 13 & 80 & 80 & 82 \\
\hline & OTHER Source Port Entropy & 9 & 58 & 77 & 69 \\
\hline & OTHER Destination Port Entropy & 55 & 72 & 79 & 72 \\
\hline \multirow[t]{3}{*}{$20 \%$} & TCP Bytes & 35 & 47 & 63 & 51 \\
\hline & TCP Flows & 31 & 44 & 58 & 40 \\
\hline & TCP Source Address Entropy & 28 & 50 & 58 & 44 \\
\hline
\end{tabular}




\begin{tabular}{|c|c|c|c|c|c|}
\hline TCP Destination Address Entropy & 7 & 44 & 69 & 51 \\
\hline TCP Source Port Entropy & 34 & 41 & 51 & 37 \\
\cline { 2 - 6 } & TCP Destination Port Entropy & 16 & 30 & 65 & 42 \\
\hline OTHER Bytes & 8 & 25 & 52 & 40 \\
\hline OTHER Flows & 8 & 73 & 73 & 69 \\
\hline & 11 & 72 & 80 & 72 \\
\cline { 2 - 6 } & OTHER Source Address Entropy & 11 & 82 & 80 & 81 \\
\cline { 2 - 6 } OTHER Destination Address Entropy & 11 & 60 & 78 & 67 \\
\cline { 2 - 6 } & OTHER Source Port Entropy & 8 & 71 & 77 & 71 \\
\cline { 2 - 6 } & OTHER Destination Port Entropy & 39 & 79 &
\end{tabular}

Table 6.29: March 8, 2009 - Wavelet Short and Low threshold results 


\begin{tabular}{|c|c|c|c|c|c|}
\hline \multirow{2}{*}{$\begin{array}{c}\text { Infection } \\
\text { Rate }\end{array}$} & \multirow[t]{2}{*}{ Feature } & \multicolumn{4}{|c|}{ Miss Count } \\
\hline & & $\begin{array}{c}\text { Static } \\
\text { Average }\end{array}$ & $\begin{array}{l}\text { Moving } \\
\text { Average } \\
\text { without } \\
\text { Outliers }\end{array}$ & $\begin{array}{l}\text { Moving } \\
\text { Average }\end{array}$ & $\begin{array}{l}\text { Weighted } \\
\text { Moving } \\
\text { Average }\end{array}$ \\
\hline \multirow[t]{12}{*}{$5 \%$} & TCP Bytes & 47 & 65 & 52 & 39 \\
\hline & TCP Flows & 50 & 65 & 46 & 42 \\
\hline & TCP Source Address Entropy & 46 & 64 & 44 & 23 \\
\hline & TCP Destination Address Entropy & 43 & 43 & 43 & 42 \\
\hline & TCP Source Port Entropy & 47 & 62 & 32 & 24 \\
\hline & TCP Destination Port Entropy & 32 & 47 & 36 & 35 \\
\hline & OTHER Bytes & 26 & 43 & 33 & 27 \\
\hline & OTHER Flows & 57 & 70 & 44 & 28 \\
\hline & OTHER Source Address Entropy & 25 & 65 & 39 & 10 \\
\hline & OTHER Destination Address Entropy & 44 & 58 & 43 & 41 \\
\hline & OTHER Source Port Entropy & 38 & 63 & 40 & 9 \\
\hline & OTHER Destination Port Entropy & 52 & 67 & 56 & 54 \\
\hline \multirow[t]{12}{*}{$10 \%$} & TCP Bytes & 40 & 48 & 68 & 53 \\
\hline & TCP Flows & 43 & 51 & 68 & 47 \\
\hline & TCP Source Address Entropy & 24 & 47 & 67 & 45 \\
\hline & TCP Destination Address Entropy & 41 & 44 & 46 & 44 \\
\hline & TCP Source Port Entropy & 25 & 48 & 65 & 33 \\
\hline & TCP Destination Port Entropy & 34 & 31 & 44 & 35 \\
\hline & OTHER Bytes & 27 & 26 & 44 & 27 \\
\hline & OTHER Flows & 13 & 42 & 70 & 40 \\
\hline & OTHER Source Address Entropy & 9 & 35 & 64 & 39 \\
\hline & OTHER Destination Address Entropy & 35 & 44 & 57 & 42 \\
\hline & OTHER Source Port Entropy & 8 & 37 & 62 & 40 \\
\hline & OTHER Destination Port Entropy & 54 & 54 & 68 & 53 \\
\hline \multirow[t]{12}{*}{$20 \%$} & TCP Bytes & 42 & 50 & 70 & 55 \\
\hline & TCP Flows & 46 & 52 & 68 & 49 \\
\hline & TCP Source Address Entropy & 26 & 49 & 69 & 46 \\
\hline & TCP Destination Address Entropy & 4 & 42 & 52 & 43 \\
\hline & TCP Source Port Entropy & 26 & 50 & 67 & 35 \\
\hline & TCP Destination Port Entropy & 4 & 34 & 46 & 36 \\
\hline & OTHER Bytes & 29 & 29 & 48 & 35 \\
\hline & OTHER Flows & 57 & 56 & 71 & 49 \\
\hline & OTHER Source Address Entropy & 23 & 40 & 58 & 39 \\
\hline & OTHER Destination Address Entropy & 64 & 37 & 51 & 49 \\
\hline & OTHER Source Port Entropy & 16 & 42 & 60 & 42 \\
\hline & OTHER Destination Port Entropy & 37 & 45 & 63 & 46 \\
\hline
\end{tabular}

Table 6.30: May 28, 2009 - Wavelet Long and Low threshold results 


\begin{tabular}{|c|c|c|c|c|c|}
\hline \multirow{2}{*}{$\begin{array}{c}\text { Infection } \\
\text { Rate }\end{array}$} & \multirow[t]{2}{*}{ Feature } & \multicolumn{4}{|c|}{ Miss Count } \\
\hline & & $\begin{array}{c}\text { Static } \\
\text { Average }\end{array}$ & $\begin{array}{c}\text { Moving } \\
\text { Average } \\
\text { without } \\
\text { Outliers }\end{array}$ & $\begin{array}{l}\text { Moving } \\
\text { Average }\end{array}$ & $\begin{array}{l}\text { Weighted } \\
\text { Moving } \\
\text { Average }\end{array}$ \\
\hline \multirow[t]{12}{*}{$75 \%$} & TCP Bytes & 63 & 63 & 67 & 62 \\
\hline & TCP Flows & 59 & 67 & 73 & 64 \\
\hline & TCP Source Address Entropy & 53 & 46 & 45 & 41 \\
\hline & TCP Destination Address Entropy & 54 & 32 & 57 & 50 \\
\hline & TCP Source Port Entropy & 60 & 53 & 56 & 51 \\
\hline & TCP Destination Port Entropy & 23 & 26 & 55 & 29 \\
\hline & OTHER Bytes & 11 & 54 & 55 & 55 \\
\hline & OTHER Flows & 6 & 61 & 62 & 51 \\
\hline & OTHER Source Address Entropy & 9 & 59 & 65 & 60 \\
\hline & OTHER Destination Address Entropy & 25 & 61 & 60 & 61 \\
\hline & OTHER Source Port Entropy & 8 & 56 & 68 & 64 \\
\hline & OTHER Destination Port Entropy & 17 & 60 & 61 & 56 \\
\hline \multirow[t]{12}{*}{$100 \%$} & TCP Bytes & 64 & 64 & 68 & 63 \\
\hline & TCP Flows & 60 & 66 & 72 & 65 \\
\hline & TCP Source Address Entropy & 54 & 47 & 46 & 42 \\
\hline & TCP Destination Address Entropy & 13 & 28 & 50 & 40 \\
\hline & TCP Source Port Entropy & 61 & 52 & 55 & 52 \\
\hline & TCP Destination Port Entropy & 24 & 27 & 52 & 30 \\
\hline & OTHER Bytes & 10 & 51 & 52 & 56 \\
\hline & OTHER Flows & 7 & 62 & 62 & 53 \\
\hline & OTHER Source Address Entropy & 9 & 54 & 64 & 56 \\
\hline & OTHER Destination Address Entropy & 22 & 64 & 62 & 61 \\
\hline & OTHER Source Port Entropy & 9 & 56 & 69 & 63 \\
\hline & OTHER Destination Port Entropy & 13 & 61 & 62 & 58 \\
\hline \multirow[t]{12}{*}{$120 \%$} & TCP Bytes & 64 & 64 & 68 & 63 \\
\hline & TCP Flows & 60 & 66 & 72 & 65 \\
\hline & TCP Source Address Entropy & 54 & 47 & 46 & 42 \\
\hline & TCP Destination Address Entropy & 12 & 22 & 44 & 39 \\
\hline & TCP Source Port Entropy & 61 & 52 & 55 & 52 \\
\hline & TCP Destination Port Entropy & 25 & 27 & 51 & 30 \\
\hline & OTHER Bytes & 10 & 51 & 52 & 56 \\
\hline & OTHER Flows & 7 & 62 & 61 & 53 \\
\hline & OTHER Source Address Entropy & 10 & 56 & 63 & 58 \\
\hline & OTHER Destination Address Entropy & 15 & 63 & 60 & 60 \\
\hline & OTHER Source Port Entropy & 10 & 57 & 69 & 63 \\
\hline & OTHER Destination Port Entropy & 9 & 57 & 56 & 55 \\
\hline
\end{tabular}

Table 6.31: January 15, 2009 - Wavelet Short and High threshold results 


\begin{tabular}{|c|c|c|c|c|c|}
\hline \multirow{2}{*}{$\begin{array}{l}\text { Infection } \\
\text { Rate }\end{array}$} & \multirow[t]{2}{*}{ Feature } & \multicolumn{4}{|c|}{ Miss Count } \\
\hline & & $\begin{array}{c}\text { Static } \\
\text { Average }\end{array}$ & $\begin{array}{c}\text { Moving } \\
\text { Average } \\
\text { without } \\
\text { Outliers } \\
\end{array}$ & $\begin{array}{l}\text { Moving } \\
\text { Average }\end{array}$ & $\begin{array}{l}\text { Weighted } \\
\text { Moving } \\
\text { Average }\end{array}$ \\
\hline \multirow[t]{12}{*}{$75 \%$} & TCP Bytes & 33 & 45 & 59 & 55 \\
\hline & TCP Flows & 33 & 48 & 65 & 50 \\
\hline & TCP Source Address Entropy & 30 & 60 & 60 & 54 \\
\hline & TCP Destination Address Entropy & 7 & 25 & 63 & 44 \\
\hline & TCP Source Port Entropy & 43 & 61 & 62 & 55 \\
\hline & TCP Destination Port Entropy & 5 & 24 & 53 & 37 \\
\hline & OTHER Bytes & 10 & 16 & 46 & 20 \\
\hline & OTHER Flows & 17 & 39 & 80 & 41 \\
\hline & OTHER Source Address Entropy & 8 & 69 & 68 & 58 \\
\hline & OTHER Destination Address Entropy & 9 & 72 & 71 & 67 \\
\hline & OTHER Source Port Entropy & 8 & 31 & 67 & 54 \\
\hline & OTHER Destination Port Entropy & 24 & 66 & 67 & 61 \\
\hline \multirow[t]{12}{*}{$100 \%$} & TCP Bytes & 32 & 46 & 58 & 42 \\
\hline & TCP Flows & 32 & 47 & 64 & 44 \\
\hline & TCP Source Address Entropy & 19 & 36 & 55 & 39 \\
\hline & TCP Destination Address Entropy & 6 & 25 & 60 & 33 \\
\hline & TCP Source Port Entropy & 44 & 62 & 64 & 50 \\
\hline & TCP Destination Port Entropy & 6 & 22 & 53 & 30 \\
\hline & OTHER Bytes & 9 & 15 & 45 & 11 \\
\hline & OTHER Flows & 10 & 40 & 79 & 40 \\
\hline & OTHER Source Address Entropy & 10 & 70 & 69 & 56 \\
\hline & OTHER Destination Address Entropy & 17 & 73 & 71 & 69 \\
\hline & OTHER Source Port Entropy & 9 & 24 & 57 & 40 \\
\hline & OTHER Destination Port Entropy & 24 & 67 & 67 & 57 \\
\hline \multirow[t]{12}{*}{$120 \%$} & TCP Bytes & 31 & 43 & 57 & 41 \\
\hline & TCP Flows & 31 & 49 & 65 & 15 \\
\hline & TCP Source Address Entropy & 17 & 45 & 55 & 39 \\
\hline & TCP Destination Address Entropy & 5 & 26 & 62 & 34 \\
\hline & TCP Source Port Entropy & 45 & 64 & 65 & 51 \\
\hline & TCP Destination Port Entropy & 5 & 25 & 53 & 29 \\
\hline & OTHER Bytes & 9 & 16 & 45 & 10 \\
\hline & OTHER Flows & 11 & 41 & 80 & 41 \\
\hline & OTHER Source Address Entropy & 11 & 41 & 66 & 50 \\
\hline & OTHER Destination Address Entropy & 11 & 72 & 70 & 70 \\
\hline & OTHER Source Port Entropy & 10 & 28 & 62 & 43 \\
\hline & OTHER Destination Port Entropy & 22 & 65 & 65 & 55 \\
\hline
\end{tabular}

Table 6.32: June 10, 2009 - Wavelet Long and High threshold results 


\subsubsection{Summary and Discussion}

The Haar Wavelet Filtering Analysis approach examines each feature and provides an indication of which features might have anomalies. There were three user-defined parameters: the size of the input data, filtering boundaries and the local variability weights for score calculation. The dataset size affects the bin width and the resolution of the filtering. A typical attack is short and $98 \%$ of the flows are less than a minute. A larger bin will have more flows, overall higher values, and will less likely to reflect quick spikes in values. Conversely, if the bin width is too small then the feature values will be small, and analysis becomes overly granular.

Input data size and filter boundaries. In the Haar Wavelet Filtering algorithm, the boundary sizes are related to input size. These boundaries define the amount of the high pass, mid pass and low pass values in filtering. Larger dataset size allows more levels to be divided into the high, medium and low regions. The experiments varying the data set sizes and filtering boundaries showed that an input size of 128 elements and emphasis on mid pass values performed the best. In this case, the bin width was 11.25 minutes and similar to what was used in literature. Larger input data sizes result in smaller bin sizes and performed worse. This can be attributed to smaller observation values and less change between time bins.

Local Variability Weights. The local variability weights are used in the deviation score calculation. The weights apply emphasis to each of the filtered values at a point of time. The high pass weights affect the sensitivity to high frequency changes and the mid pass weights affect the mid pass frequency changes. The experiments conducted examined a strong bias and a small bias towards high pass values. Each feature has its own independent analysis and the results from all of the features were examined. The weights with a strong bias generally had lower miss counts. The feature, which isolated the injected malicious flows, consistently had 
smaller miss counts. A possible reason for this is because the mid pass values are not as meaningful as the high pass values. Most of the anomalous flows were less than one minute long, in 11.25 min bins, and quick changes in features would be more evident.

The Haar Wavelet Filtering analysis was able to isolate all of the infected bins in at least one of the feature analysis. As the infection rate and duration increased, the approach performed better. The major drawback of this approach is that every feature is a separate independent analysis and can result in several independent, unrelated results. In practice, it would be very difficult to determine which feature(s) detecting the actual intrusions, and which are missing them.

\subsection{Evaluation of Hybrid PCA - Haar Wavelet Filtering Analysis}

This section outlines the results obtained from running the experiments with the Hybrid PCA- Haar Wavelet Filtering analysis approach outlined in section 5.3.4 and concludes with a brief discussion.

\subsubsection{Hybrid Analysis and Pure data}

This section examines the results of the Hybrid PCA - Haar Wavelet Filtering analysis applied to a dataset with no infections. The results presented are for January 6, 2009, which is representative of all 16 dates. Figure 6.20 shows the delta scores across all ten test sets. Unlike the Modified PCA and Haar Wavelet Filtering approaches, the Hybrid PCA - Haar Wavelet Filtering approach does not have extreme values to influence the average or flatten the visual plot. As a result, pre-algorithm filtering is not needed in the Hybrid PCA - Haar Wavelet Filtering approach. The threshold analysis was applied to the average of the test sets or footprint. 
Similar to previous approaches, as shown in Figure 6.20, regardless of the threshold technique, the algorithm will suggest anomalous time bins.

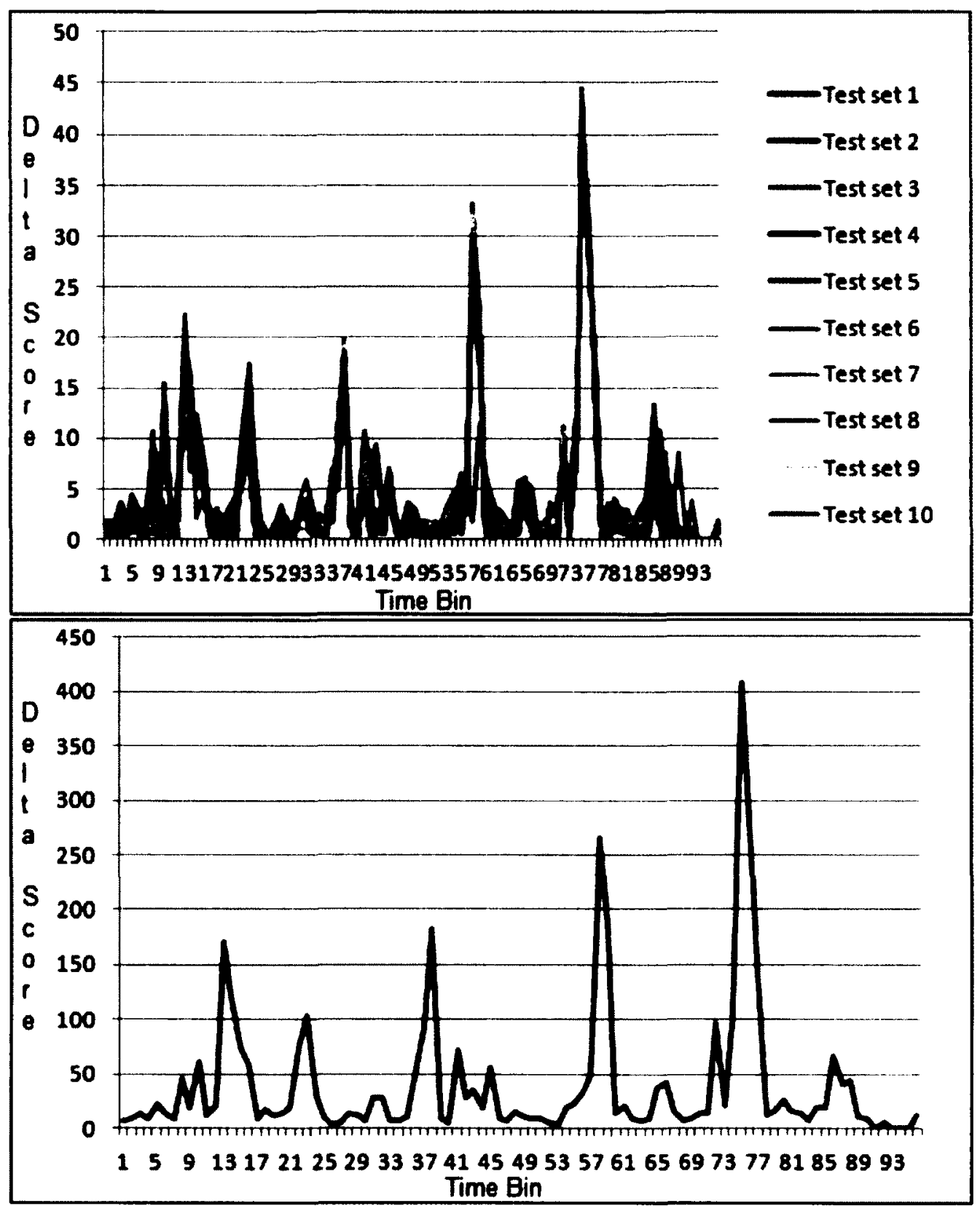

Figure 6.200: January 6, 2009 - Hybrid Delta scores (top) and Average Delta scores (bottom) 


\subsubsection{Short and Low Infection}

This section examines the results of the Hybrid PCA - Haar Wavelet filtering analysis experiments performed on a low infection rate in a short period of time. The four dates for this particular scenario, outlined in Table 5.2, all had consistent behaviour. As a result, for simplicity, only March 8, 2009 is discussed. Figures 6.21 illustrates the footprints observed with default parameters conducted on test sets of $5 \%, 10 \%$ and $20 \%$ infection ratios respectively (See Table 5.4 for a list of default conditions). The infected time bins were during bins 88,89 and 90 and the plots clearly picked up the infected times. Table 6.33 lists the hit/miss scores and the hit/miss counts measured for each feature. The default threshold method determines a miss rate of about $30 \%$. The other threshold methods range from $4 \%$ to $39 \%$, see Section 6.3 .6 for details

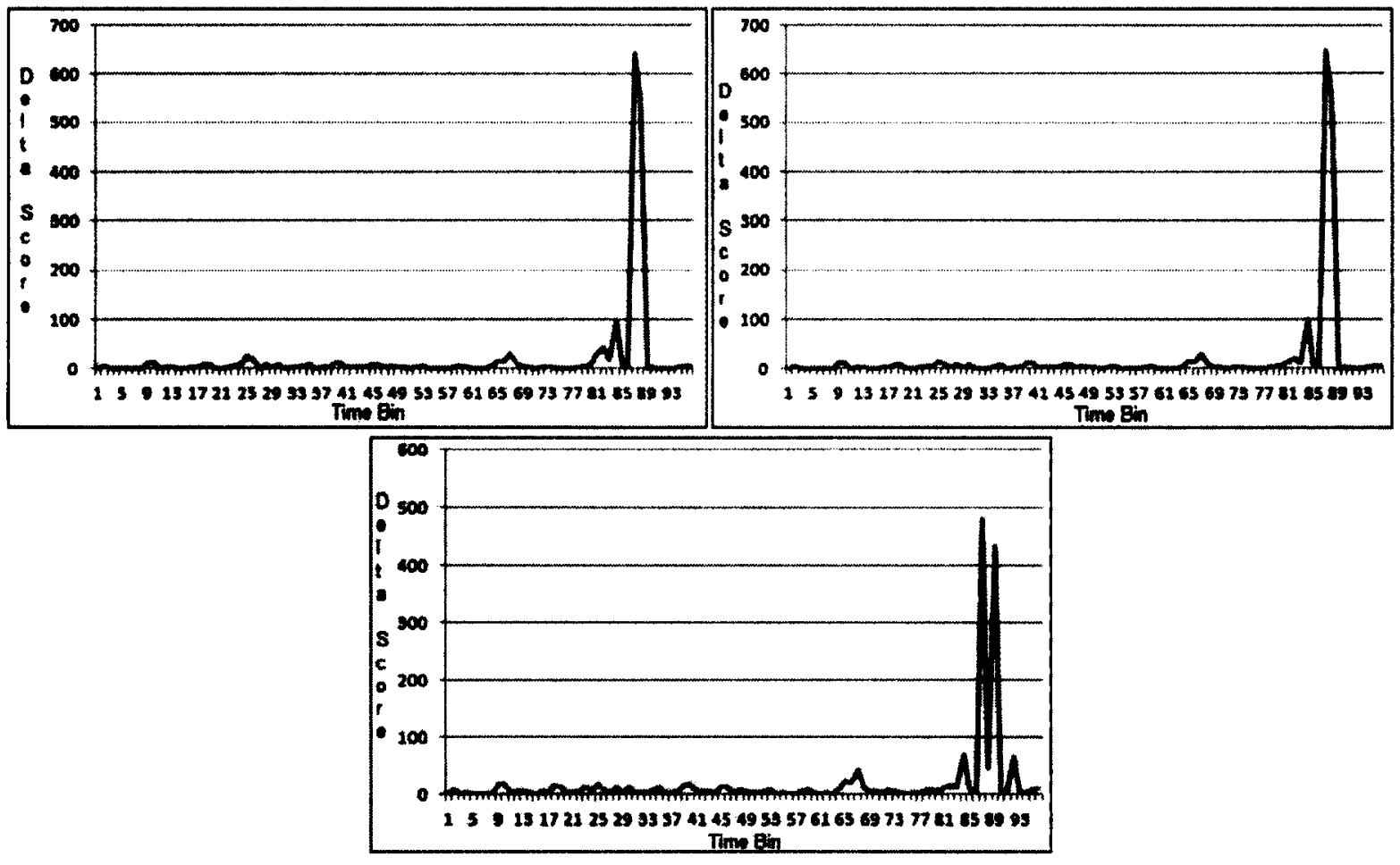

Figure 6.211: March 8, 2009 - Hybrid Delta Scores of 5\% (top left), 10\% (top right), 20\%(bottom) infection rates 


\begin{tabular}{|c|c|c|c|}
\hline Infection Rate & Score & Hit Count (\% Hit) & Miss Count (\% Miss) \\
\hline $5 \%$ & 1 & $62(65 \%)$ & $34(35 \%)$ \\
\hline $10 \%$ & 1 & $62(65 \%)$ & $34(35 \%)$ \\
\hline $20 \%$ & 1 & $63(66 \%)$ & $36(34 \%)$ \\
\hline
\end{tabular}

Table 6.33: March 8, 2009 - Hybrid experiment results

\subsubsection{Long and Low Infection}

This section examines the results of the Hybrid PCA - Haar Wavelet filtering analysis experiments performed on a low infection rate in a short period of time. The four dates for this particular scenario, outlined in Table 5.2, all had consistent behaviour. As a result, for simplicity, only May 28, 2009 is discussed. Figures 6.22 illustrates the footprints observed with default parameters conducted on test sets of $5 \%, 10 \%$ and $20 \%$ infection ratios respectively (See Table 5.4 for a list of default conditions). The infected time bins were during bins $61-71$ and the plots clearly picked up the infected times. Table 6.34 lists the hit/miss scores and the hit/miss counts measured for each feature. The default threshold method determines a miss rate of about $20 \%$. The other threshold methods range from $11 \%$ to $40 \%$, see Section 6.3 .6 for details 

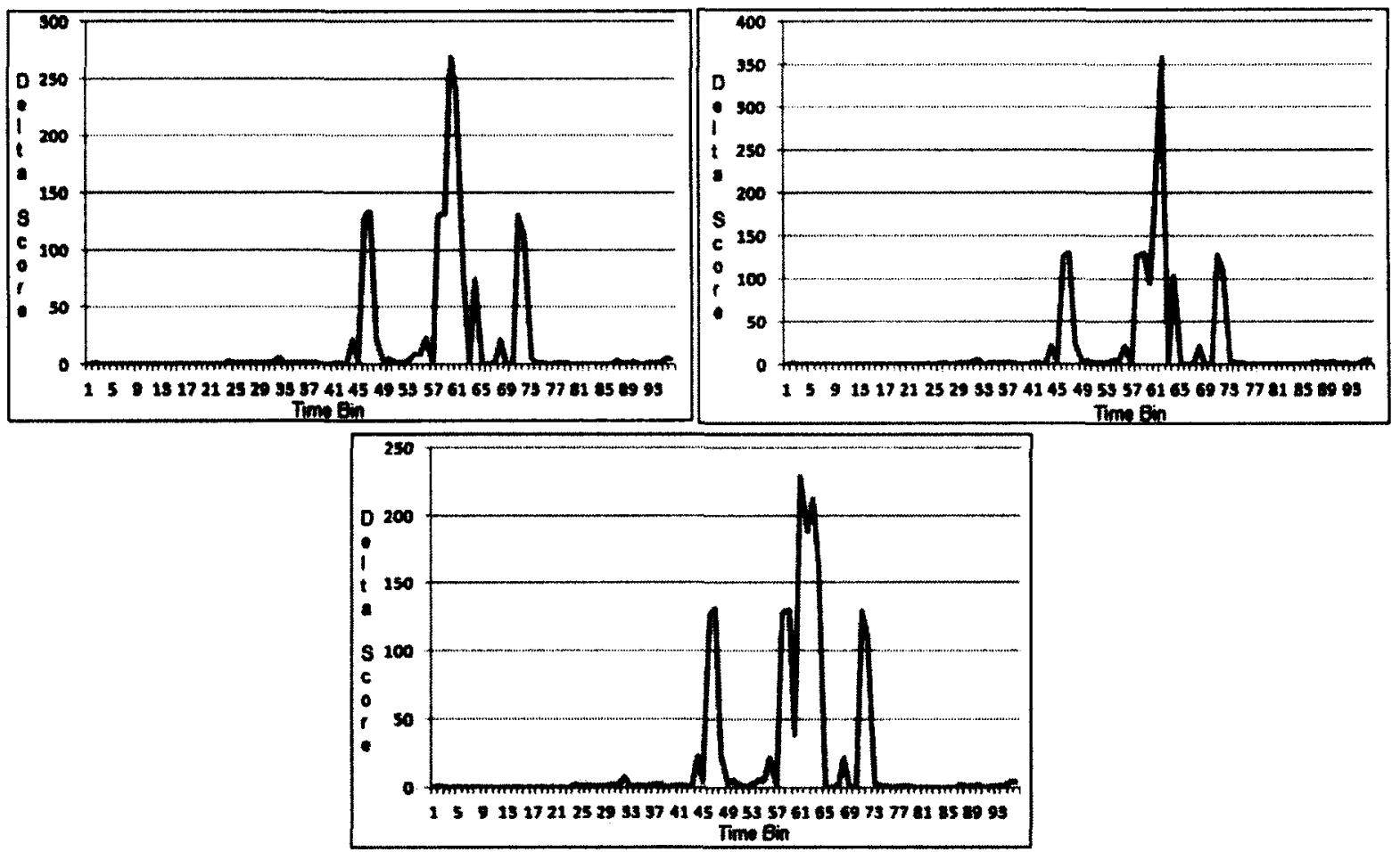

Figure 6.222: May 28, 2009 - Hybrid Delta Scores of 5\% (top left), 10\% (top right), 20\% (bottom) infection rates

\begin{tabular}{|c|c|c|c|}
\hline Infection Rate & Score & Hit Count (\% Hit) & Miss Count (\% Miss) \\
\hline $5 \%$ & 1 & $71(74 \%)$ & $25(26 \%)$ \\
\hline $10 \%$ & 0 & $77(80 \%)$ & $19(20 \%)$ \\
\hline $20 \%$ & 2 & $77(80 \%)$ & $19(20 \%)$ \\
\hline
\end{tabular}

Table 6.34: May 28, 2009 - Hybrid experiment results

\subsubsection{Short and High Infection}

This section examines the results of the Hybrid PCA - Haar Wavelet filtering analysis experiments performed on a low infection rate in a short period of time. The four dates for this particular scenario, outlined in Table 5.2, all had consistent behaviour. As a result, for simplicity, only January 15, 2009 is discussed. Figures 6.23 illustrates the footprints observed with default parameters conducted on test sets of $75 \%, 100 \%$ and $120 \%$ infection ratios respectively (See Table 5.4 for a list of default conditions). The infected time bins were during bins $83,84,85,86$ and the plots clearly picked up the infected times. Table 6.35 lists the 
hit/miss scores and the hit/miss counts measured for each feature. The default threshold method determines a miss rate of about $35 \%$. The other threshold methods range from $4 \%$ to $38 \%$, see Section 6.3.6 for details
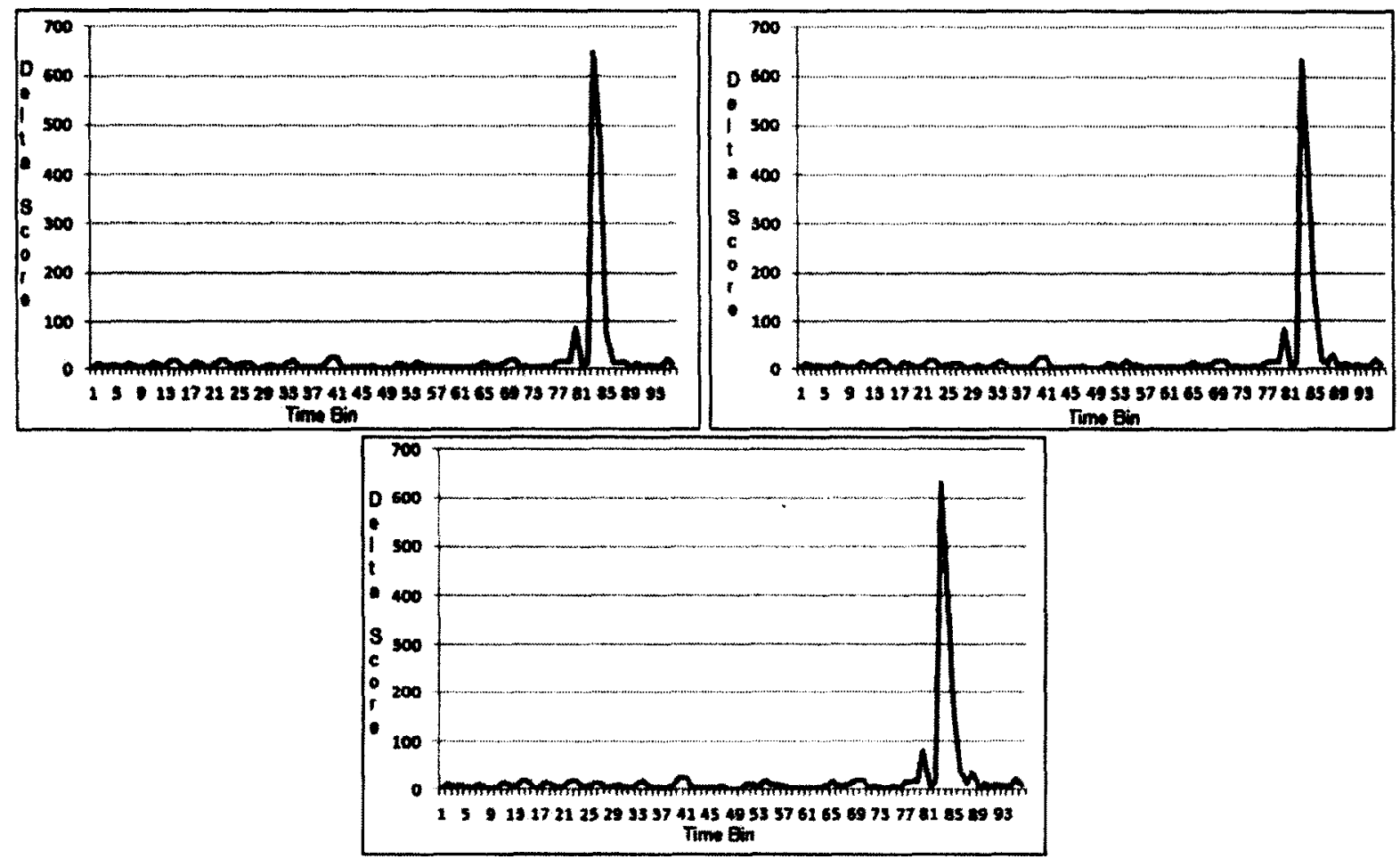

Figure 6.233:January 15, 2009 - Hybrid Delta Scores of 75\% (top left), 100\% (top right), 120\%(bottom) infection rates

\begin{tabular}{|c|c|c|c|}
\hline Infection Rate & Score & Hit Count (\% Hit) & Miss Count (\% Miss) \\
\hline $75 \%$ & 1 & $61(64 \%)$ & $35(36 \%)$ \\
\hline $100 \%$ & 2 & $62(65 \%)$ & $34(35 \%)$ \\
\hline $120 \%$ & 3 & $62(65 \%)$ & $34(35 \%)$ \\
\hline
\end{tabular}

Table 6.35: January 15, 2009 - Hybrid experiment results

\subsubsection{Long and High Infection}

This section examines the results of the Hybrid PCA - Haar Wavelet filtering analysis experiments performed on a low infection rate in a short period of time. The four dates for this particular scenario, outlined in Table 5.2, all had consistent behaviour. As a result, for simplicity, only June 10, 2009 is discussed. Figures 6.24 illustrates the footprints observed with 
default parameters conducted on test sets of $75 \%, 100 \%$ and $120 \%$ infection ratios respectively (See Table 5.4 for a list of default conditions). The infected time bins were during bins $22-32$ and the plots clearly picked up the infected times. Table 6.36 lists the hit/miss scores and the hit/miss counts measured for each feature. The default threshold method determines a miss rate of about $25 \%$. The other threshold methods range from $6 \%$ to $41 \%$, see Section 6.3 .6 for details

\begin{tabular}{|c|c|c|c|}
\hline Infection Rate & Score & Hit Count (\% Hit) & Miss Count (\% Miss) \\
\hline $75 \%$ & 3 & $73(76 \%)$ & $23(24 \%)$ \\
\hline $100 \%$ & 3 & $76(79 \%)$ & $20(21 \%)$ \\
\hline $120 \%$ & 2 & $70(73 \%)$ & $26(27 \%)$ \\
\hline
\end{tabular}

Table 6.36: June 10, 2009 - Hybrid experiment results
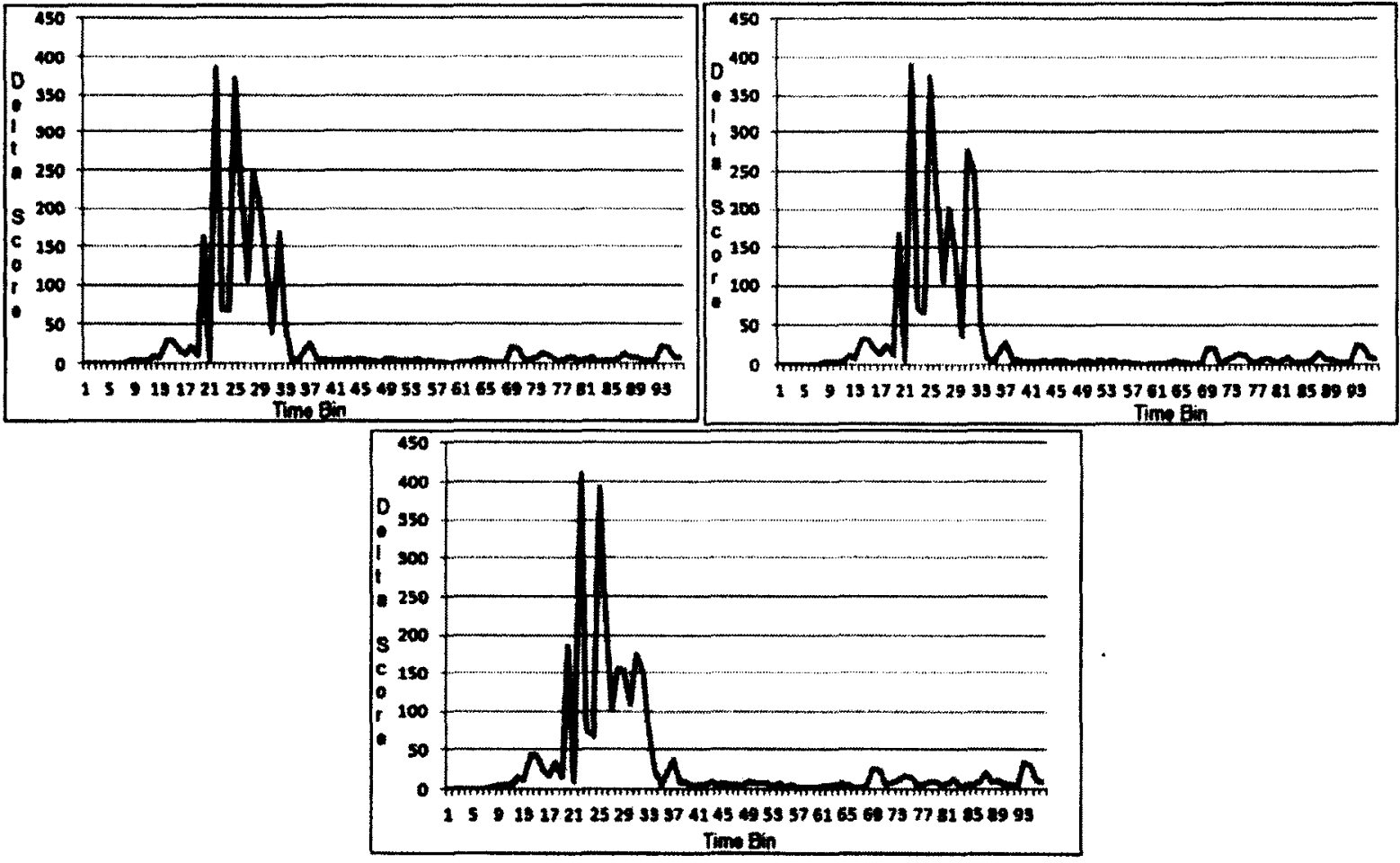

Figure 6.244:June 10, 2009 - Hybrid Delta Scores of 75\% (top left), 100\% (top right), 120\% (bottom) infection rates 


\subsubsection{Threshold Techniques and Hybrid PCA - Haar Wavelet filtering Analysis}

Each of the experiments performed used four different threshold techniques. The results listed in tables $6.37-6.40$ summarize the evaluation measurements for threshold techniques on the specified dates. The delta scores contain sets of distinctly high scores and are represented by spikes when plotted. The static average was found to perform the best.

\begin{tabular}{|c|c|c|c|c|c|}
\hline $\begin{array}{l}\text { Infection } \\
\text { Rate }\end{array}$ & Measurement & Average & $\begin{array}{l}\text { Moving Average } \\
\text { without outliers }\end{array}$ & $\begin{array}{l}\text { Moving } \\
\text { Average }\end{array}$ & $\begin{array}{l}\text { Weighted } \\
\text { Average }\end{array}$ \\
\hline \multirow{3}{*}{$5 \%$} & Score & 1 & 1 & 1 & 1 \\
\cline { 2 - 6 } & Hit Count & 90 & 62 & 63 & 60 \\
\cline { 2 - 6 } & Miss Count & 6 & 34 & 33 & 36 \\
\hline \multirow{3}{*}{$10 \%$} & Score & 1 & 1 & 1 & 1 \\
\cline { 2 - 6 } & Hit Count & 92 & 62 & 63 & 59 \\
\cline { 2 - 6 } & Miss Count & 4 & 34 & 33 & 37 \\
\hline \multirow{2}{*}{$20 \%$} & Score & 2 & 1 & 66 & 1 \\
\cline { 2 - 6 } & Hit Count & 89 & 63 & 33 & 36 \\
\cline { 2 - 6 } & Miss Count & 7 & 36 & & \\
\hline
\end{tabular}

Table 6.37: March 8, 2009 - Hybrid threshold results

\begin{tabular}{|c|c|c|c|c|c|}
\hline $\begin{array}{c}\text { Infection } \\
\text { Rate }\end{array}$ & Measurement & Average & $\begin{array}{c}\text { Moving Average } \\
\text { without outliers }\end{array}$ & $\begin{array}{c}\text { Moving } \\
\text { Average }\end{array}$ & $\begin{array}{c}\text { Weighted } \\
\text { Average }\end{array}$ \\
\hline \multirow{3}{*}{$5 \%$} & Score & 2 & -1 & -21 & -1 \\
\cline { 2 - 6 } & Hit Count & 84 & 77 & 56 & 69 \\
\cline { 2 - 6 } & Miss Count & 12 & 19 & 40 & 27 \\
\hline \multirow{3}{*}{$10 \%$} & Score & 2 & 0 & -20 & 0 \\
\cline { 2 - 6 } & Hit Count & 84 & 77 & 56 & 74 \\
\cline { 2 - 6 } & Miss Count & 12 & 19 & 40 & 24 \\
\hline \multirow{3}{*}{$20 \%$} & Score & 4 & 2 & -18 & 2 \\
\cline { 2 - 6 } & Hit Count & 85 & 77 & 57 & 76 \\
\cline { 2 - 6 } & Miss Count & 11 & 19 & 39 & 23 \\
\hline
\end{tabular}

Table 6.38: May 28, 2009 - Hybrid threshold results 


\begin{tabular}{|c|c|c|c|c|c|}
\hline $\begin{array}{c}\text { Infection } \\
\text { Rate }\end{array}$ & Measurement & Average & $\begin{array}{c}\text { Moving Average } \\
\text { without outliers }\end{array}$ & $\begin{array}{c}\text { Moving } \\
\text { Average }\end{array}$ & $\begin{array}{c}\text { Weighted } \\
\text { Average }\end{array}$ \\
\hline \multirow{3}{*}{$75 \%$} & Score & 4 & 1 & 1 & 1 \\
\cline { 2 - 6 } & Hit Count & 92 & 61 & 58 & 59 \\
\cline { 2 - 6 } & Miss Count & 4 & 35 & 38 & 37 \\
\hline \multirow{2}{*}{$100 \%$} & Score & 2 & 2 & 2 & 2 \\
\cline { 2 - 6 } & Hit Count & 91 & 62 & 59 & 60 \\
\cline { 2 - 6 } & Miss Count & 5 & 34 & 37 & 36 \\
\hline \multirow{2}{*}{$120 \%$} & Score & 3 & 2 & 2 & 2 \\
\cline { 2 - 6 } & Hit Count & 91 & 62 & 39 & 59 \\
\cline { 2 - 6 } & Miss Count & 5 & 34 & 37 & 37 \\
\hline
\end{tabular}

Table 6.39: January 15, 2009 - Hybrid threshold results

\begin{tabular}{|c|c|c|c|c|c|}
\hline $\begin{array}{c}\text { Infection } \\
\text { Rate }\end{array}$ & Measurement & Average & $\begin{array}{c}\text { Moving Average } \\
\text { without outliers }\end{array}$ & $\begin{array}{c}\text { Moving } \\
\text { Average }\end{array}$ & $\begin{array}{c}\text { Weighted } \\
\text { Average }\end{array}$ \\
\hline \multirow{2}{*}{$75 \%$} & Score & 9 & 3 & 2 & 4 \\
\cline { 2 - 6 } & Hit Count & 90 & 73 & 60 & 66 \\
\cline { 2 - 6 } & Miss Count & 6 & 23 & 36 & 30 \\
\hline \multirow{2}{*}{$100 \%$} & Score & 9 & 3 & 2 & 5 \\
\cline { 2 - 6 } & Hit Count & 90 & 76 & 60 & 68 \\
\cline { 2 - 6 } & Miss Count & 6 & 20 & 36 & 28 \\
\hline \multirow{2}{*}{$120 \%$} & Score & 10 & 2 & 5 & 5 \\
\cline { 2 - 6 } & Hit Count & 88 & 73 & 41 & 28 \\
\cline { 2 - 6 } & Miss Count & 8 & 26 & 58 & \\
\hline
\end{tabular}

Table 6.40: June 10, 2009 - Hybrid threshold results 


\subsubsection{Summary and Discussion}

The Hybrid PCA - Haar Wavelet Filtering analysis is an approach that considers all features and has few input parameters. This method draws upon the strengths of the Modified PCA approach and the Haar Wavelet Filtering analysis. It examines all features using Principle Component Analysis and selects the single best principle component for anomalous subspace creation. It uses the one top component to build the anomalous space. As shown in Modified PCA experimentation, this approach performs best with this dataset. Next, the Haar Wavelet Filtering analysis is applied with input data size of 128. As shown in Haar Wavelet Filtering analysis, applying filter boundaries, and local variability weightings, which have a bias towards high frequency values, performs best. In experimentation, it was able to constantly detect the injections. This approach was able to detect all infected time bins; regardless of the how infected a time bins was or how many time bins were infected (See Table 6.41 for a summary of the differences between the approaches). As the intensity or duration of anomalous behaviour increased, the algorithm performed better.

\begin{tabular}{|l|c|c|c|}
\hline \multicolumn{1}{|c|}{ Characteristic } & $\begin{array}{c}\text { Modified PCA } \\
\text { Approach }\end{array}$ & Haar Wavelet Approach & Hybrid Approach \\
\hline $\begin{array}{l}\text { Number of input } \\
\text { dimensions }\end{array}$ & All & 1 & All \\
\hline $\begin{array}{l}\text { Number of output } \\
\text { dimensions }\end{array}$ & 1 & All (1 for each dimension) & 1 \\
\hline $\begin{array}{l}\text { Number of tunable } \\
\text { user parameters }\end{array}$ & Fair. & $\begin{array}{c}\text { Ranges from Good to poor, } \\
\text { depending on the } \\
\text { dimension. }\end{array}$ & Good. \\
\hline $\begin{array}{l}\text { Overall detection } \\
\text { performance }\end{array}$ & & 3 & 0 \\
\hline
\end{tabular}

Table 6.41: Summary of differences between algorithms 


\subsection{Threshold Techniques Discussion}

In order to automate the anomaly detection analysis, four different threshold techniques were examined. One threshold technique considered the entire dataset and the three remaining threshold techniques considered sliding window. The static average calculates a single cutoff line, while the dynamic techniques form a cutoff function. Depending on the algorithm and the nature of the scores, the techniques performed differently. The Modified PCA algorithm creates scores that have a lot of highs and lows. Also, the extreme highs skew the average value. In this approach, the static average performed the worst. The dynamic average approaches, which considered only a small subset of consecutive values, had much better miss counts. The Haar wavelet approach and the Hybrid approach have distinct regions of high values. Using a sliding window through regions of low values is meaningless. Dynamic thresholds are overly granular in these approaches. The static average was able to calculate an appropriate cutoff to isolate these distinct spikes. By examining the output plots, it is evident that the threshold techniques choice is directly dependant on the algorithm applied. 


\subsection{Conclusion}

This chapter summarizes the thesis and presents conclusions. Future work and directions for further research are also discussed.

\subsection{Conclusions and Summary}

This thesis introduces a hybrid intrusion detection approach based on anomaly detection methods. The hybrid approach involves using well known statistical analysis and spectral analysis techniques to provide the network administrator time slices of potential network traffic intrusions. Unlike existing Intrusion Detection Systems, which primarily use signature based investigation, a novel technique which used as adaptive model based on flow characteristics was presented. The statistical analysis technique, Modified Principle Component Analysis, considers data trends and creates a new set of axis to better describe the dataset. It projects data onto the anomalous subspace and provides an anomalous score for each time bin. Two parameters investigated in this research were the number of time shifts and how the components were selected for the anomalous subspace. It was concluded that the features in the Kyoto2006+ dataset are highly correlated. Building a subspace of components that contains $99 \%$ of the normal space performed the best in experimentation. The difference of observation values between time bins changes a fair amount and it was determined that considering more than one time shift degraded performance. For anomaly detection, the algorithm calculated a Square Prediction Error (SPE) score for an indication of how anomalous each time bin was. Experiments were conducted with various time bin infection rates and over a short and long number of infected time bins. The PCA approach was able to produce higher SPE score for 
infected time slices; however, these values were not large enough to stand out. The scores were not one of the dominant spikes when plotted.

Secondly, the spectral analysis technique used Haar Wavelet Filtering analysis to determine abrupt changes in the data over time. It provides a score to show how anomalous each time bin may be. Three parameters investigated in this research were the number of time bins fed into the algorithm, the filtering boundaries and the frequency component weightings for score calculation. Due to the nature of the Haar Wavelet Filtering, the input size had to be a power of two. Considering the Kyoto2006+ dataset volume of observations, the optimal number of input time bins, through experimentation, was determined to be 128 . The time bins were large enough to be meaningful. The spectral analysis algorithm modeled high pass values to represent the start /end time and the mid pass values to represent the duration of malicious behaviour. Through experimentation, boundaries where the high pass region consisted of the first two levels, the mid pass region consisted of the next three levels and the low pass regions consisted of the remaining levels performed best. These specific boundaries resulted create a preference on high pass frequency values. The local variability weights applied a further bias to each of the filtered values to determine a single scalar score. Once again, the weights heavier on high pass performed best. Considering this trend, it can be concluded that in the Kyoto2006+ dataset has significant fluctuation of feature values and are best isolated by high frequency analysis. The Haar Wavelet Filtering approach performed independent feature analysis and produces several independent scores. The experiments examined different days in week, different times, various infections ratios and various amount of infected time bins. Under all circumstances tested, the analysis was able to isolate the infected time bins in at least one of the feature analysis. When examining individual experiment results, it was observed that at least one of the feature results 
identified the infected time bins ideally; yet other features were completely wrong. Without knowing where the anomalies are, it would be very difficult to determine which features accurately picked up the anomalies. The Haar Wavelet Filtering approach performed much better than the Modified PCA approach; however, it is very difficult to determine which of the feature plots to consider.

Both the Modified PCA and the Haar Wavelet Filtering approach detected the infected time bins. Both approaches had their strengths and drawbacks. The Hybrid analysis draws upon the strengths of each approach and combines them in a complementary fashion. The Hybrid analysis considers the entire feature set, as in modified PCA, and performs an in depth analysis, as in Haar Wavelet Filtering. Similar to the previous two algorithms, the hybrid approach was tested on several dates, using various infection rates and different number of consecutive infected time bins. In all circumstances, the Hybrid approach detected all the infected times bins. It produced a single output score that distinctly identified regions of anomalous behaviour.

An automated detection approach was investigated and various threshold techniques were applied to the output scores of each of the algorithms. In the modified PCA approach, where the plotted output had many regions of high spikes, it was observed that moving thresholds performed best. Applying threshold techniques to smaller portions to determine local spikes performed much better than considering the entire dataset. Conversely, the hybrid and wavelet analysis had a distinct and localized region of high values. The remaining scores were all very low. In the experiments using these algorithms, the static average performed the best. One threshold approach did not always perform the best. Depending on which algorithm is applied, one threshold technique would perform better the others. 
In conclusion, applying the modified PCA approach on the Kyoto2006+ dataset performed modestly well. Applying Haar Wavelet Filtering on the Kyoto2006+ better detected the injected anomalous flows; however, due to the amount of independent feature analysis, it is not very practical. The Hybrid approach used the strengths of both previous approaches. This algorithm was applied on the Kyoto2006+ dataset and it was always able to consistently identify the anomalous time bins. It can be concluded that the Hybrid PCA - Haar Wavelet Filtering approach performed the best.

\subsection{Future Work}

Directions for future research are discussed next.

- Investigate techniques to isolate specific hosts or sources within time bins. Our approach suggests a specific infected time bin, and it would be very useful to provide an indication of which hosts within this time behaved anomalously.

- Devise techniques to isolate specific characteristics, which contributed to the anomaly. The major benefit of the Haar Wavelet approach was that it specifically listed the feature interest. Due to the nature of the Modified PCA approach and the Hybrid approach, semantic meaning is lost.

- Investigate multiple infected time bins regions. Our analysis examined the base cases of a single infected region of a short or long time. It would be useful to examine how the algorithms perform one datasets with multiple infected regions.

- Apply anomaly detection algorithms on various datasets. There are a number of different types of dataset available and each have their limitations. Some datasets have higher traffic volume and different are characterized by different features.

- Apply the profiling phase to involve to TCP and UDP network protocols. Due to availability of data, this research involved dividing between TCP and OTHER, all remaining, protocols. When considering a different dataset, the profiling could be divided between TCP and UDP network protocols. 
This research was built upon existing research. It starts with known statistical and spectral analysis techniques and builds upon them. The result of these algorithms provides an indication of which time bins may have malicious behaviour, and it would be beneficial to provide the network administrator more information as to which sources are infected, or to which features are creating the anomaly. Furthermore, the experiments showed promising result under base cases with the novel labeled dataset. It would be interesting to apply the anomaly detection algorithms on different dataset with higher data volume, different features, and different protocol partitions. This research provides was a good starting point in comparing the performance of the different anomaly detection algorithms in network traffic data. 


\section{References}

[1] J. M. Estevez-Tapiador, P. Garcia-Teodoro, and J. E. Diaz-Verdejo, "Anomaly detection methods in wired networks: a survey and taxonomy," Computer Commun., vol. 27, no. 16, pp. 1569-1584, 2004.

[2] Introduction to Cisco ISO NetFlow - A Technical Overview. [Online]. Available at: http://www.cisco.com/en/US/prod/collateral/iosswrel/ps6537/ps6555/ps6601/prod_white_ paper0900aecd80406232.html [Last Accessed: March 2012].

[3] Netflow version 9. [Online]. Available at: http://www.cisco.com/en/US/products/ps6645/products_ios_protocol_option_home.html [Last Accessed: March 2012]

[4] The Bro Security Network Monitor. [Online]. Available at: http://www.bro-ids.org [Last Accessed: March 2012]

[5] M. Campos and B. Milenova, "Creation and deployment of data mining-based intrusion detection systems in oracle database $10 \mathrm{~g}$," in Proc. 4th Int. Conf. Mach. Learning and Applicat., pp. 8-15, 2005.

[6] D. Brauckhoff, K. Salamatian, and M. May, "Applying PCA for traffic anomaly detection: Problems and solutions," in Proc. of the IEEE INFOCOM, pp. 2866-2870, 2009.

[7] P. Barford, J. Kline, D. Plonka, and A. Ron, "A signal analysis of network traffic anomalies," in Proc. of the 2nd ACM SIGCOMM Workshop on Internet measurement, pp. 71-82, 2002.

[8] J. Song, H. Takakura, Y. Okabe, M. Eto, D. Inoue, and K. Nakao, "Statistical analysis of honeypot data and building of Kyoto 2006+ dataset for NIDS evaluation," in Proc. of the 1st Workshop on Building Anal. Datasets and Gathering Experience Returns for Security, pp. 29-36, 2011.

[9] J. Zhang and M. Zulkernine, "A hybrid network intrusion detection technique using random forests," in Proc. of the 1st Int. Conf. Availability, Reliability and Security, pp. 815, 2006.

[10] Y.T. Chan, Wavelet Basics. Boston, MA: Kluwer Academic Publishers, 1995.

[11] B. Vidakovic and P. Mueller. (1994). Wavelets for kids [Online]. Available at: www2.isye.gatech.edu/ -brani/wp/kidsA.pdf [Last Accessed: March 2012]

[12] J. Goswami and A. Chan, Fundamental of Wavelets - Theory, Algorithms and Applications. Toronto, Canada: Wiley, 1999. 
[13] A. Webb, Statistical Pattern Recognition, $2^{\text {nd }}$ ed. West Sussex, England: Wiley, 2002.

[14] W. Stallings, Data and Computer Communications, $9^{\text {th }}$ ed. Toronto, Canada: Prentice Hall, 2011.

[15] H. Ringberg, A. Soule, J. Rexford, and C. Diot, "Sensitivity of PCA for traffic anomaly detection," in ACM SIGMETRICS Performance Evaluation Review, vol. 35, no. 1, pp. 109-120, 2007.

[16] A. Lakhina, M. Crovella, and C. Diot, "Diagnosing network-wide traffic anomalies," in ACM SIGCOMM Computer Commun. Review, vol. 34, pp. 219-230, 2004.

[17] KDD 1999 Cup Data. [Online]. Available at: http://kdd.ics.uci.edu/databases/kddcup99/kddcup99.html [Last Accessed: March 2012]

[18] F. Wanner, "Anomaly Analysis using Host-behavior Clustering," M.S. thesis, ETH-Swiss Federal Institute of Technology Zurich, 2007.

[19] K. Nyalkalkar, S. Sinhay, M. Bailey, and F. Jahanian, "A comparative study of two network-based anomaly detection methods," in Proc. of the IEEE INFOCOM, pp. 176$180,2011$.

[20] L. Smith, A Tutorial on Principal Components Analysis [Online]. Available at: http://www.sccg.sk/ haladova/principal_components.pdf [Last Accessed: March 2012]

[21] R. Dunia and S. J. Qin, "Multi-dimensional fault diagnosis using a subspace approach," in American Control Conf., 1997.

[22] S. Jungsuk, H. Takakura, Y. Okabe, D. Inoue, E. T. O. Masashi, and K. Nakao, "A Comparative Study of Unsupervised Anomaly Detection Techniques Using Honeypot Data," in IEICE Trans. Inform. and Syst., vol. 93, no. 9, pp. 2544-2554, 2010.

[23] J. Song, H. Takakura, and Y. Kwon, "A Generalized Feature Extraction Scheme to Detect 0-Day Attacks via IDS Alerts," in Int. Symp. Applicat. and the Internet, pp. 55-61, 2008.

[24] SNORT. [Online]. Available at: http://www.snort.org/ [Last Accessed: March 2012]

[25] L. Zheng, P. Zou, Y. Jia, and W. Han, "Traffic Anomaly Detection and Containment Using Filter-Ary-Sketch," in Procedia Engineering, vol. 29, pp. 4297-4306, 2012.

[26] J. Song, H. Takakura, Y. Okabe, and K. Nakao. (2011, Aug.). "Toward a more practical unsupervised anomaly detection system." Inform. Sciences. [Online]. Available: http://www.sciencedirect.com/science/article/pii/S0020025511004245 [Last Accessed: March 2012] 
[27] A. Dainotti, A. Pescapé, and G. Ventre, "Wavelet-based detection of DoS attacks," in Proc. of the IEEE Global Telecommun. Conf. (GLOBECOM), pp. 1-6, 2006.

[28] M. Salagean, "Real network traffic anomaly detection based on Analytical Discrete Wavelet Transform," in Proc. of the 12th Int. Conf. Optimization of Elect. and Electron. Equipment (OPTIM), pp. 926-931, 2010.

[29] M. Salagean and I. Firoiu, "Anomaly detection of network traffic based on Analytical Discrete Wavelet Transform," in Proc. of the 8th Int. Conference Commun., pp. 49-52, 2010.

[30] A. Lakhina, M. Crovella, and C. Diot, "Characterization of network-wide anomalies in traffic flows," in Proc. of the 4th ACM SIGCOMM conf. on Internet measurement, pp. 201-206, 2004.

[31] B. Whitehead, C. H. Lung, and P. Rabinovitch, "Tracking per-flow state-Binned Duration Flow Tracking," in Proc. of the Int. Symp. Performance Evaluation of Comput. and Telecommun. Syst. (SPECTS), pp. 73-80, 2010.

[32] C. Callegari, S. Giordano, and M. Pagano, "Application of Wavelet Packet Transform to Network Anomaly Detection," in Next Generation Teletraffic and Wired/Wireless Advanced Networking, pp. 246-257, 2008.

[33] A. Soule, K. Salamatian, and N. Taft, "Combining filtering and statistical methods for anomaly detection," in Proc. of the 5th ACM SIGCOMM conf. on Internet Measurement, pp. 31-31, 2005.

[34] Flex. [Online]. Available at: http://www.adobe.com/products/flex.html [Last Accessed: March 2012]

[35] Apache Tomcat. [Online]. Available at: http://tomcat.apache.org/ [Last Accessed: March 2012] 